\title{
One-particle (improper) States in Nelson's Massless Model
}

\author{
Alessandro Pizzo
}

\begin{abstract}
In the one-particle sector of Nelson's massless model, the one-particle states are constructed for an arbitrarily small infrared cutoff in the interaction term of the Hamiltonian of the system. The performed method is a constructive one which exploits only regular perturbation theory, by a suitable iteration scheme. The disappearance of one-particle states is showed in the limit of no infrared regularization. Constructive features, as regularity in some parameters, are also inquired.
\end{abstract}

\section{Introduction}

In this paper we treat some spectral problems in a model describing quantum mechanical matter locally interacting with the quantized relativistic field of scalar massless bosons. Such a model was rigorously studied, in the case of massive bosons, by Nelson [Ne], who removed the ultraviolet cutoff in the interaction. Nowadays it is widely considered a toy model for analyzing infrared aspects of radiation theory. The underlying conjecture is that the model retains some of the infrared features of Q.E.D., in spite of the various approximations made: the charge is not described by a field (no pair production), an ultraviolet cutoff is (generally) imposed on the interaction, the "photons" are scalar and the "electron" is a spin less non-relativistic particle.

In a rigorous analysis of radiation theory, the zero photon mass implies nontrivial mathematical problems at the level of spectrum properties, which are not avoidable for a satisfactory explanation of radiative phenomena at low energies. In this respect many papers have been recently devoted to a rigorous analysis of binding and resonances ([B.F.S], [Ge], [G.L.L], [L.M.S], [Ar]), scattering of photons and relaxation to the ground state for isolated atoms ([Sp], [D.Ge], [Ge], [F.G.S]).

In this paper we are concerned with the translation invariant Nelson's massless model restricted to only one non-relativistic particle interacting with the boson (scalar) field: the so-called "one-particle sector". The aim is to clarify, in an interacting and physically non-trivial model, the phenomenon of the disappearance of a properly defined mass-shell for the electron in Q.E.D. [Sc], [Bu], when no infrared regularization is performed. Such an analysis is also a prerequisite for a rigorous treatment of the counterpart of "Compton scattering" in the given scalar model.

It is worth-while to stress that, though important indications come from solvable infrared models, like non-relativistic Q.E.D. in dipole approximation (also called Pauli-Fierz model [P.F], [Bl] ) or a simplified version of Nelson's model it- 
self (see [Fr.1] page 27), the answers one gets for the mass-shell problem are not satisfactory, because the adopted approximations are too strong. For this reason the analysis of the full interacting Nelson's model turns out to be a relevant nonperturbative step to analyze some (limited) aspects of the mass-shell problem in Q.E.D.

Mathematically, the mass-shell problem is formalized as the absence of the "one-particle subspace" in the Hilbert space of the system; the one-particle subspace is that one generated by vectors on which the Hamiltonian $H$ acts as a function of the total momentum $\mathbf{P}$ of the system (the considered model is covariant under translation). The states in the one-particle space (if it exists with some properties) describe a free particle alone in the world, with an expected non-relativistic dispersion for small momenta. For Nelson's massless model, this subspace exists in the Hilbert space of the system as long as an infrared cutoff on the interaction is imposed, as proved in [Fr.1] and in the present paper. Our main concerns are therefore: to study the fate of one-particle states when the infrared cutoff is removed, and to get a control on the involved vectors for an arbitrarily small infrared cutoff.

More technically, such a study concerns the limiting behavior of the ground states of the Hamiltonians $H_{\mathbf{P}}$, at a fixed total momentum $\mathbf{P}$ and acting on a copy of the boson Fock space, when no infrared cutoff is introduced in the interaction term. For this purpose we use an iterative procedure different from the operatorial renormalization group developed for analogous problems in [B.F.S] and [Ch]. The method we use provides the construction of the ground state for the Hamiltonians $H_{\mathbf{P}, \sigma}$, obtained from $H_{\mathbf{P}}$ by an (arbitrarily small) infrared cutoff $\sigma$ in the interaction. The method is based on the analytic perturbation [R.S], it works for small values of the coupling constant and in a neighborhood of $\mathbf{P}=0$, corresponding to a ratio $|\mathbf{P}| / m$ strictly less than 1 , where $m$ is the Hamiltonian parameter corresponding to the non-relativistic particle. It exploits the "smallness" of the variation of the interaction term when we slightly modify the infrared (energy) scale. By the same method we can prove the strong convergence of the ground state for some Hamiltonians $H_{\mathbf{P}, \sigma}^{w}$, acting on the Fock space and obtained from $H_{\mathbf{P}, \sigma}$ through a $\mathbf{P}$-dependent coherent transformation of the boson variables, already known from [Fr.1]. This result easily implies the weak limit to the zero vector of the ground eigenvector of the original Hamiltonian $H_{\mathbf{P}, \sigma}$, for $\sigma \rightarrow 0$. It also prevents any massshell construction by glueing states corresponding to different values of $\mathbf{P}$; in fact, because of the inequivalence, in the limit $\sigma \rightarrow 0$, of the coherent transformations for different $\mathbf{P}$, it turns out to be physically meaningless (see [Fr.1], page 53).

The fundamental results were already discovered by Fröhlich [Fr.1], [Fr.2], through a non-perturbative method inspired by Glimm and Jaffe [G.J], which is not constructive. By the iterated analytic perturbation, the one-particle states are studied here in terms of the ground eigenvectors of related transformed Hamiltonians $\left(H_{\mathbf{P}, \sigma}^{w}\right)$. The new vectors are more regular in their dependence on parameters like the infrared cutoff and the total momentum. Such a characterization of oneparticle states, for arbitrarily small infrared cutoffs, is a key ingredient for a rather 
complete description of the asymptotic dynamics, both for the massless field and for the charged particle [Pi].

In conclusion, the constructive method provides a more explicit control and then more insight into the physical content of the limit states. Indeed, in this framework, regular perturbation theory becomes effective, even though the limiting situation is that of a non-isolated eigenvalue and therefore seemingly not accessible through such a method. Simple questions can be answered, at least partially, by ordinary perturbation techniques; for instance: how two ground states at different cutoff or at different $\mathbf{P}$ are related, in which sense and to what extent the expected regularity, of certain physical quantities, is conserved under the removal of the infrared cutoff.

\section{Definition of the model}

The physical system consists of a non-relativistic spin less quantum particle of mass $m$, linearly coupled to a quantized relativistic scalar boson field, which is massless and real.

The non-relativistic particle is described by position and momentum variables with usual canonical commutation rules (c.c.r.)

$$
\left[x_{l}, p_{j}\right]=i \delta_{l, j} \quad(\hbar=1) .
$$

The (scalar) boson field, which we will call photon field, at time $t=0$ is

$$
A(0, \mathbf{y})=\frac{1}{\sqrt{2 \pi}^{3}} \cdot \int\left(a^{\dagger}(\mathbf{k}) e^{-i \mathbf{k} \cdot \mathbf{y}}+a(\mathbf{k}) e^{i \mathbf{k} \cdot \mathbf{y}}\right) \frac{d^{3} k}{\sqrt{2|\mathbf{k}|}},
$$

$(c=\hbar=1)$, where $a^{\dagger}(\mathbf{k}), a(\mathbf{k})$ are standard creation and annihilation operatorvalued tempered distributions obeying the c.c.r.

$$
\begin{aligned}
{\left[a(\mathbf{k}), a^{\dagger}(\mathbf{q})\right] } & =\delta^{3}(\mathbf{k}-\mathbf{q}) \\
{[a(\mathbf{k}), a(\mathbf{q})] } & =\left[a^{\dagger}(\mathbf{k}), a^{\dagger}(\mathbf{q})\right]=0 .
\end{aligned}
$$

The spatial translations are implemented by the total momentum

$$
\mathbf{P}:=\mathbf{p}+\int \mathbf{k} a^{\dagger}(\mathbf{k}) a(\mathbf{k}) d^{3} k .
$$

The dynamics of the system is generated by the covariant (under translation, $[H, \mathbf{P}]=0)$ Hamiltonian

$$
H:=\frac{\mathbf{p}^{2}}{2 m}+g \int_{0}^{\kappa}\left(a(\mathbf{k}) e^{i \mathbf{k} \cdot \mathbf{x}}+a^{\dagger}(\mathbf{k}) e^{-i \mathbf{k} \cdot \mathbf{x}}\right) \frac{d^{3} k}{\sqrt{2}|\mathbf{k}|^{\frac{1}{2}}}+H^{p h}
$$

where $\kappa$ is an ultraviolet cutoff (the integration bounds throughout the paper are referred to the radial part of $\mathbf{k}, g$ is the coupling constant and $H^{p h}$ is the free Hamiltonian of the photon field $\left.H^{p h}:=\int|\mathbf{k}| a^{\dagger}(\mathbf{k}) a(\mathbf{k}) d^{3} k\right)$. 
The Hilbert space of the system is $\mathrm{H}=L^{2}\left(R^{3}, d^{3} x\right) \otimes F$ where $F$ is the Fock space with respect to the creation and annihilation operator-valued distributions $\left\{a^{\dagger}(\mathbf{k}), a(\mathbf{k})\right\}$ : an element $\psi$ of $\mathrm{H}$ is a sequence $\left\{\psi^{n}\right\}_{n \in N}$ of functions on $R^{3(n+1)}$ with $\|\psi\|<\infty$, where

$$
\|\psi\|^{2}=\sum_{n=0}^{\infty} \int \overline{\psi^{n}\left(\mathbf{x}, \mathbf{k}_{1}, \ldots, \mathbf{k}_{n}\right)} \psi^{n}\left(\mathbf{x}, \mathbf{k}_{1}, \ldots, \mathbf{k}_{n}\right) d^{3} k_{1} \ldots d^{3} k_{n} d^{3} x
$$

and each $\psi^{n}\left(\mathbf{x}, \mathbf{k}_{1}, \ldots, \mathbf{k}_{n}\right)$ is symmetric in $\mathbf{k}_{1}, \ldots, \mathbf{k}_{n}$. The $n=0$ component corresponds to the tensor product of the vacuum subspace of $F$ with the nonrelativistic particle space $L^{2}\left(R^{3}\right)$.

Standard results about $H$ and $P$ :

i) The operators

$$
\mathbf{P}=\mathbf{p}+\int \mathbf{k} a^{\dagger}(\mathbf{k}) a(\mathbf{k}) d^{3} k
$$

are essentially self-adjoint (e.s.a.) in

$$
D:=\bigvee_{n \in N} h \otimes \psi^{n}
$$

which is the set of the finite linear combinations of vectors of wave functions $h(\mathbf{x}) \psi^{n}\left(\mathbf{k}_{1}, \ldots, \mathbf{k}_{n}\right)$, where $h(\mathbf{x}) \in S\left(R^{3}\right)$ (the space of Schwartz test functions), $\psi^{n}\left(\mathbf{k}_{1}, \ldots, \mathbf{k}_{n}\right) \in S_{s}\left(R^{3 n}\right)$ is symmetric in its variables. Since $\mathbf{p} \equiv-i \frac{d}{d \mathbf{x}}$ and $\int \mathbf{k} a^{\dagger}(\mathbf{k}) a(\mathbf{k}) d^{3} k$ are e.s.a. in $S\left(R^{3}\right)$ and $\bigvee_{n \in N} \psi^{n}$ respectively, the result easily follows for the $\mathbf{P}$ operators. The spectrum of each component of $\mathbf{P}$ is the real axis, the spectral measure is absolutely continuous with respect to the Lebesgue measure.

ii) The interaction term in the Hamiltonian is infinitesimal small with respect to

$$
H_{0}:=\frac{\mathbf{p}^{2}}{2 m}+H^{p h} .
$$

Therefore $H$ is bounded from below, it is e.s.a. in $D$ and its self-adjointness domain (s.a.d.), $D(H)$, coincides with $D\left(H_{0}\right)$ (s.a.d. of $\left.H_{0}\right)$.

iii) The groups $e^{i \mathbf{a} \cdot \mathbf{P}}$ and $e^{i \tau H}\left(\tau, a^{i} \in R\right)$ commute.

iv) The joint spectral decomposition of the Hilbert space with respect to the $\mathbf{P}$ operators is $\mathrm{H}=\int{ }^{\oplus} \mathrm{H}_{\mathbf{P}} d^{3} P$, where $\mathrm{H}_{\mathbf{P}}$ is a copy of $F$.

Indeed to the improper eigenvectors of the $\mathbf{P}$ operators, $\Phi_{\mathbf{P}}^{n}$, where

$$
\begin{gathered}
\Phi_{\mathbf{P}}^{n}\left(\mathbf{x}, \mathbf{k}_{1}, \ldots, \mathbf{k}_{n}\right):=(2 \pi)^{-\frac{3}{2}} e^{i\left(\mathbf{P}-\mathbf{k}_{1}-\cdots-\mathbf{k}_{n}\right) \cdot \mathbf{x}} \varphi_{\mathbf{P}}^{n}\left(\mathbf{k}_{1}, \ldots, \mathbf{k}_{n}\right) \\
\varphi_{\mathbf{P}}^{n}\left(\mathbf{k}_{1}, \ldots, \mathbf{k}_{n}\right) \in S_{s}\left(R^{3 n}\right),
\end{gathered}
$$


we can relate a natural scalar product:

$$
\left(\Phi_{\mathbf{P}}^{\prime n}, \Phi_{\mathbf{P}}^{m}\right)=\delta_{n, m} \int \overline{\varphi_{\mathbf{P}}^{\prime n}\left(\mathbf{k}_{1}, \ldots, \mathbf{k}_{n}\right)} \varphi_{\mathbf{P}}^{m}\left(\mathbf{k}_{1}, \ldots, \mathbf{k}_{n}\right) d^{3} k_{1} \ldots d^{3} k_{n}
$$

The vector space $\overline{\bigvee_{n \in N} \Phi_{\mathbf{P}}^{n}}$ is defined as the closure of the finite linear combinations of the wave functions $\Phi_{\mathbf{P}}^{n}\left(\mathbf{x}, \mathbf{k}_{1}, \ldots, \mathbf{k}_{n}\right)$ in the norm which arises from the scalar product above. Starting from this space, we uniquely define the linear application

$$
I_{\mathbf{P}}: \varlimsup_{n \in N} \Phi_{\mathbf{P}}^{n} \rightarrow F^{b}
$$

by the prescription:

$$
\begin{aligned}
& I_{\mathbf{P}}\left(\Phi_{\mathbf{P}}^{n}\left(\mathbf{x}, \mathbf{k}_{1}, \ldots, \mathbf{k}_{n}\right)\right) \\
& =\frac{1}{\sqrt{n !}} \int b^{\dagger}\left(\mathbf{k}_{1}\right) \ldots b^{\dagger}\left(\mathbf{k}_{n}\right) \varphi_{\mathbf{P}}^{n}\left(\mathbf{k}_{1}, \ldots, \mathbf{k}_{n}\right) d^{3} k_{1} \ldots d^{3} k_{n} \psi_{0}
\end{aligned}
$$

where $b(\mathbf{k}), b^{\dagger}(\mathbf{k})$, which formally correspond to $a(\mathbf{k}) e^{i \mathbf{k} \cdot \mathbf{x}}, a^{\dagger}(\mathbf{k}) e^{-i \mathbf{k} \cdot \mathbf{x}}$, are annihilation and creation operator-valued tempered distributions in the Fock space $F^{b} \cong F$, and $\psi_{0}$ is the related vacuum. The given norm for $\Phi_{\mathbf{P}}^{n}$ is equal to $\left\|I_{\mathbf{P}}\left(\Phi_{\mathbf{P}}^{n}\right)\right\|_{F}\left(\|\cdot\|_{F}\right.$ is the Fock norm). The application $I_{\mathbf{P}}$ is onto and unitary.

v) Since $[H, \mathbf{P}]=0$, we have that $H=\int H_{\mathbf{P}} d^{3} P$, where $H_{\mathbf{P}}: \mathrm{H}_{\mathbf{P}} \rightarrow \mathrm{H}_{\mathbf{P}}$ is e.s.a. in $D^{b}:=\bigvee_{n \in N} \Phi_{\mathbf{P}}^{n}$; in terms of the variables $\mathbf{P}, b(\mathbf{k}), b^{\dagger}(\mathbf{k})$, the operator $H_{\mathbf{P}}$ is written as follows:

$$
H_{\mathbf{P}}=\frac{\left(\mathbf{P}^{p h}-\mathbf{P}\right)^{2}}{2 m}+g \int_{0}^{\kappa}\left(b(\mathbf{k})+b^{\dagger}(\mathbf{k})\right) \frac{d^{3} k}{\sqrt{2|\mathbf{k}|}}+H^{p h}
$$

being $H^{p h} \equiv \int|\mathbf{k}| b^{\dagger}(\mathbf{k}) b(\mathbf{k}) d^{3} k$ and $\mathbf{P}^{p h} \equiv \int \mathbf{k} b^{\dagger}(\mathbf{k}) b(\mathbf{k}) d^{3} k$ when applied on the fiber spaces $\mathrm{H}_{\mathbf{P}}$.

Survey of results

Our first concern (Section 1) is to single out a sequence of infrared cutoffs, $\left\{\sigma_{j}\right\}$, $\sigma_{j}=\kappa \epsilon^{\frac{j}{2}}, j \in N$ (natural numbers), $0<\epsilon<\left(\frac{1}{5}\right)^{8}$, and to construct, for $g$ uniform in $j$, the ground eigenvectors $\psi_{\mathbf{P}}^{\sigma_{j}}$ of the Hamiltonians $H_{\mathbf{P}, \sigma_{j}}$ acting on $\mathrm{H}_{\mathbf{P}} \cong F^{b}$ and with the infrared cutoff $\sigma_{j}$ in the interaction term, namely

$$
H_{\mathbf{P}, \sigma_{j}}=\frac{\left(\mathbf{P}^{p h}-\mathbf{P}\right)^{2}}{2 m}+g \int_{\sigma_{j}}^{\kappa}\left(b(\mathbf{k})+b^{\dagger}(\mathbf{k})\right) \frac{d^{3} k}{\sqrt{2|\mathbf{k}|}}+H^{p h}
$$

where $\mathbf{P}$ belongs to $\Sigma \equiv\left\{\mathbf{P}:|\mathbf{P}| \leq \frac{m}{20}\right\}$. The constraint on $\Sigma$ reflects the mixed character of the model, which forces to restrict the physical region of the total momentum to the set $\{\mathbf{P}:|\mathbf{P}|<m\}$; the adopted more restrictive constraint, $\left\{\mathbf{P}:|\mathbf{P}| \leq \frac{m}{20}\right\}$, is only due to technical reasons. The law in the infrared sequence, 
$\left\{\sigma_{j}\right\}$, derives from the requirement to have, step by step, a relative interaction $\left(\Delta H_{\mathbf{P}}\right)_{\sigma_{j+1}}^{\sigma_{j}}$ (defined below) "of the same order" as the gap at least. The restrictive constraint on $\epsilon$ plays a role in the proof of Theorem 2.3 in Section 2; in Section 1 the natural constraint, $0<\epsilon<1$, works. Our proofs require in general a small ratio $\frac{\kappa}{m}$. Concerning terminology, we use the term ground eigenvector to be precise about normalization (the vectors $\psi_{\mathbf{P}}^{\sigma_{j}}$, obtained in the iteration, are not normalized) and because a phase fixing is implicit in the used procedure and for the desired results. We will generally use the term ground state to indicate only the corresponding ray; by the term "unique ground eigenvector" we mean that the corresponding eigenvalue is non-degenerate.

In constructing the ground eigenvectors $\left\{\psi_{\mathbf{P}}^{\sigma_{j}}\right\}$, the underlying idea is to break the interaction and to construct the vector $\psi_{\mathbf{P}}^{\sigma_{j+1}}$ in terms of $\psi_{\mathbf{P}}^{\sigma_{j}}$ by iteration of the analytic perturbation [R.S]. The "small" and analytic perturbation for the Hamiltonian $H_{\mathbf{P}, \sigma_{j}}$ is represented by the difference of the interaction terms

$$
\left(\Delta H_{\mathbf{P}}\right)_{\sigma_{j+1}}^{\sigma_{j}}:=H_{\mathbf{P}, \sigma_{j+1}}-H_{\mathbf{P}, \sigma_{j}}=g \int_{\sigma_{j+1}}^{\sigma_{j}}\left(b(\mathbf{k})+b^{\dagger}(\mathbf{k})\right) \frac{d^{3} k}{\sqrt{2|\mathbf{k}|}}
$$

at subsequent infrared cutoffs and at fixed coupling constant $g$.

In developing this technique, the tensorial structure of the Fock space is crucial: it means that if the Hilbert complex space $h$ is given as a direct sum $h_{1} \oplus h_{2}$, then the bosonic Hilbert space $F$ over $h, F(h)$, is isomorphic to $F_{1} \otimes F_{2}$, where $F_{1}$ is the Fock space over $h_{1}$ and $F_{2}$ is the Fock space over $h_{2}$.

The technique essentially relies on the comparison between the resolvents of the Hamiltonians $H_{\mathbf{P}, \sigma_{j}}$ and $H_{\mathbf{P}, \sigma_{j+1}}$; it recursively uses the Kato-Rellich theorem on the analytic perturbation of isolated eigenvalues (of self-adjoint operators) to relate the corresponding ground eigenvectors $\psi_{\mathbf{P}}^{\sigma_{j}}$ and $\psi_{\mathbf{P}}^{\sigma_{j+1}}$.

At each step two pieces of information are required:

1) a lower bound for the gap of the Hamiltonian $H_{\mathbf{P}, \sigma_{j}}$ restricted to the subspace

$$
F_{\sigma_{j+1}}^{+}:=F(h), \quad h:=L^{2}\left(R^{3} \backslash B_{\sigma_{j+1}}, d^{3} k\right), \quad B_{\sigma_{j+1}}:=\left\{\mathbf{k}:|\mathbf{k}|<\sigma_{j+1}\right\} ;
$$

2) an estimate of the difference, $\left(\Delta H_{\mathbf{P}}\right)_{\sigma_{j+1}}^{\sigma_{j}}:=H_{\mathbf{P}, \sigma_{j+1}}-H_{\mathbf{P}, \sigma_{j}}$, between two subsequent infrared cutoff Hamiltonians; we need that it is small with respect to $\left.H_{\mathbf{P}, \sigma_{j}}\right|_{F_{\sigma_{j+1}}^{+}}$in a generalized sense, in order to expand the spectral projection of $\left.H_{\mathbf{P}, \sigma_{j+1}}\right|_{F_{\sigma_{j+1}}^{+}}$, on the ground eigenvalue, in a perturbative series in terms of the resolvent of $\left.H_{\mathbf{P}, \sigma_{j}}\right|_{F_{\sigma_{j+1}}^{+}}$and of the difference $\left(\Delta H_{\mathbf{P}}\right)_{\sigma_{j+1}}^{\sigma_{j}}$.

The requirement 1 ) is provided by Lemma 1.1 , where we study the operator $H_{\mathbf{P}, \sigma_{j}}$ applied to the subspace $F_{\sigma_{j+1}}^{+}$. The result is that, under the constructive hypotheses for $\left\{\sigma_{j}\right\}$ and $\Sigma$, if $\psi_{\mathbf{P}}^{\sigma_{j}}$ is the unique ground eigenvector of $\left.H_{\mathbf{P}, \sigma_{j}}\right|_{F_{\sigma_{j}}}$ of energy $E_{\mathbf{P}}^{\sigma_{j}}$ with gap bigger than $\frac{\sigma_{j}}{2}$, then $\left.H_{\mathbf{P}, \sigma_{j}}\right|_{F_{\sigma_{j+1}}^{+}}$has unique ground eigenvector $\psi_{\mathbf{P}}^{\sigma_{j}} \otimes \psi_{0}$ ( $\psi_{0}$ vacuum state) with a gap larger than $\frac{3}{5} \sigma_{j+1}$. 
The meaning of this result is the following. When the operator $H_{\mathbf{P}, \sigma_{j}}$, initially considered on $F_{\sigma_{j}}^{+}$, is applied on the larger space $F_{\sigma_{j+1}}^{+}$keeping the interaction fixed above the cutoff $\sigma_{j}$, new further terms appear; however, the contribution of the new terms is, roughly speaking, positive. More precisely, it turns out that:

- the ground state is essentially the same as for $\left.H_{\mathbf{P}, \sigma_{j}}\right|_{F_{\sigma_{j}}^{+}}$;

- the new gap is of order $\sigma_{j+1}$.

The requirement 2) is provided by Lemma 1.3, in which, given the estimate for the gap provided in Lemma 1.1 and for properly chosen values of $E_{j+1} \in \mathcal{C}$ (complex numbers), we can establish that the resolvent

$$
\frac{1}{\left.H_{\mathbf{P}, \sigma_{j+1}}\right|_{F_{\sigma_{j+1}}^{+}}-E_{j+1}}
$$

has a series expansion in terms of $\frac{1}{\left.H_{\mathbf{P}, \sigma_{j}}\right|_{F_{\sigma_{j+1}}^{+}}-E_{j+1}}$ and the difference $\left(\Delta H_{\mathbf{P}}\right)_{\sigma_{j+1}}^{\sigma_{j}}$, at small, but uniform in $j$, coupling constant $g$. Except for the relevant estimate

$$
\left\|\int f(\mathbf{k}) b(\mathbf{k}) d^{3} k \psi\right\| \leq\left(\int \frac{|f(\mathbf{k})|^{2}}{|\mathbf{k}|} d^{3} k\right)^{\frac{1}{2}}\left\|H^{p h^{\frac{1}{2}}} \psi\right\|
$$

(where the expression on the right side is supposed to be well defined) and for an ordinary factorization (see also [B.F.S]) in the series expansion of the resolvent, the result is only due to a crucial consideration based on the joint spectral decomposition of commuting observables.

Then we obtain the main result of the section which consists in the definition of $\psi_{\mathbf{P}}^{\sigma_{j+1}}$ by the pertubation of $\psi_{\mathbf{P}}^{\sigma_{j}}\left(\psi_{\mathbf{P}}^{\sigma_{j}}\right.$ identified with $\psi_{\mathbf{P}}^{\sigma_{j}} \otimes \psi_{0}$, vector in $\left.F_{\sigma_{j+1}}^{+}\right)$ under some hypotheses on $\psi_{\mathbf{P}}^{\sigma_{j}}$; namely the result is:

Theorem 1.4 Under the constructive hypotheses and for $g$ sufficiently small, if $\psi_{\mathbf{P}}^{\sigma_{j}}$ is the unique ground eigenvector of $\left.H_{\mathbf{P}, \sigma_{j}}\right|_{F_{\sigma_{j}}^{+}}$with gap larger than $\frac{\sigma_{j}}{2}$, $\left.H_{\mathbf{P}, \sigma_{j+1}}\right|_{F_{\sigma_{j+1}}^{+}}$has a unique ground eigenvector $\psi_{\mathbf{P}}^{\sigma_{j+1}}$ of energy $E_{\mathbf{P}}^{\sigma_{j+1}}$ and the corresponding gap is bigger than $\frac{\sigma_{j+1}}{2}$; the (unnormalized) vector $\psi_{\mathbf{P}}^{\sigma_{j+1}}$ is so defined

$$
\psi_{\mathbf{P}}^{\sigma_{j+1}}:=P_{\sigma_{j+1}} \psi_{\mathbf{P}}^{\sigma_{j}}=-\frac{1}{2 \pi i} \oint \frac{1}{H_{\mathbf{P}, \sigma_{j+1}}-E_{j+1}} d E_{j+1} \psi_{\mathbf{P}}^{\sigma_{j}}
$$

where $E_{j+1} \in \mathcal{C}$ and $\left|E_{j+1}-E_{\mathbf{P}}^{\sigma_{j}}\right|=\frac{11}{20} \sigma_{j+1}$.

According to this theorem, it turns out that $\psi_{\mathbf{P}}^{\sigma_{j+1}}$ is given by $\psi_{\mathbf{P}}^{\sigma_{j}}$ plus a finite $g$-dependent remainder so that $\left\|\psi_{\mathbf{P}}^{\sigma_{j+1}}\right\| \geq c\left\|\psi_{\mathbf{P}}^{\sigma_{j}}\right\|$ where $0<c<1$, provided $g$ is sufficiently small. Because of this result and the spectral features of $\psi_{\mathbf{P}}^{\sigma_{j+1}}$ (gap, non-degeneracy), the same operation can be repeated for the next infrared cutoff. Then we can construct the sequence $\left\{\psi_{\mathbf{P}}^{\sigma_{j}}\right\}$, by iteration, starting from $\psi_{\mathbf{P}}^{\sigma_{0}} \equiv \psi_{0}$. 
In Section 2 we deal with the problem of the convergence of $\left\{\psi_{\mathbf{P}}^{\sigma_{j}}\right\}$ and we are forced to discuss a related sequence $\left\{\phi_{\mathbf{P}}^{\sigma_{j}}\right\}$. Mathematically, the convergence of $\left\{\psi_{\mathbf{P}}^{\sigma_{j}}\right\}$ involves a problem of perturbation of eigenvalues at the threshold of the continuous spectrum, more specifically the perturbation of the ground energy of the Hamiltonian $(\mathbf{P} \in \Sigma)$

$$
H_{\mathbf{P}}^{0}:=\frac{\left(\mathbf{P}^{p h}-\mathbf{P}\right)^{2}}{2 m}+H^{p h}
$$

If the exponent of $|\mathbf{k}|$ in the interaction term of the Hamiltonian $H_{\mathbf{P}}$

$$
g \int_{0}^{\kappa}\left(b(\mathbf{k})+b^{\dagger}(\mathbf{k})\right) \frac{d^{3} k}{\sqrt{2|\mathbf{k}|}}
$$

were larger than $-\frac{1}{2}$, the norm estimates for the resolvents would be sufficient not only to construct the sequence $\left\{\psi_{\mathbf{P}}^{\sigma_{j}}\right\}$ but also to gain the convergence. The local interaction (up to the ultraviolet cutoff $\kappa$ ) of the relativistic field yields the exponent $-\frac{1}{2}$. It is a limiting case for the existence of the ground state in the following sense:

- the ground state exists in the Fock space $F^{b}\left(\cong \mathrm{H}_{\mathbf{P}}\right)$ for arbitrarily small infrared cutoff in the interaction;

- when the infrared cutoff is removed, it requires non-Fock coherent representations of the variables $\left\{b(\mathbf{k}), b^{\dagger}(\mathbf{k})\right\}$, which are also inequivalent at different $\mathbf{P}$.

Therefore the strategy is to properly transform the Hamiltonians $H_{\mathbf{P}, \sigma_{j}}$ and to study the sequence of ground eigenvectors of the so obtained Hamiltonians acting on $F^{b}$. The known coherent transformation (in this respect see [Fr.1]) is re-obtained thanks to a heuristic proof based on a virial type argument ${ }^{1}$. Namely, starting from the assumption of a ground state "coherent in the infrared region", such an argument works out the representation given by the following intertwiner

$$
W(\nabla E(\mathbf{P})):=\exp \left[-g \int_{0}^{\kappa} \frac{b(\mathbf{k})-b^{\dagger}(\mathbf{k})}{|\mathbf{k}|(1-\widehat{\mathbf{k}} \cdot \nabla E(\mathbf{P}))} \frac{d^{3} k}{\sqrt{2|\mathbf{k}|}}\right]
$$

where $\nabla E(\mathbf{P})$ is the gradient of the ground energy (as a function of the total momentum $\mathbf{P}$ ) which is well defined as proved in [Fr.2], at least except a set of measure zero.

Taking care of the above expression we turn to consider the transformed Hamiltonians

$$
H_{\mathbf{P}, \sigma_{j}}^{w}:=W_{\sigma_{j}}\left(\nabla E^{\sigma_{j}}(\mathbf{P})\right) H_{\mathbf{P}, \sigma_{j}} W_{\sigma_{j}}^{\dagger}\left(\nabla E^{\sigma_{j}}(\mathbf{P})\right)
$$

\footnotetext{
${ }^{1} \mathrm{I}$ am indebted to G. Morchio for having suggested to me this effective argument and for many discussions and advice.
} 
where

$$
W_{\sigma_{j}}\left(\nabla E^{\sigma_{j}}(\mathbf{P})\right):=\exp \left[-g \int_{\sigma_{j}}^{\kappa} \frac{b(\mathbf{k})-b^{\dagger}(\mathbf{k})}{|\mathbf{k}|\left(1-\widehat{\mathbf{k}} \cdot \nabla E^{\sigma_{j}}(\mathbf{P})\right)} \frac{d^{3} k}{\sqrt{2|\mathbf{k}|}}\right]
$$

and $E_{\mathbf{P}}^{\sigma_{j}}$ is the ground energy of $H_{\mathbf{P}, \sigma_{j}}$.

Then we realize that $H_{\mathbf{P}, \sigma_{j}}^{w}$ can be put in the following "canonical" form

$$
\begin{aligned}
\frac{1}{2 m} & \left(\Pi_{\mathbf{P}, \sigma_{j}}-\frac{1}{\left\|\phi_{\mathbf{P}}^{\sigma_{j}}\right\|^{2}} \cdot\left(\phi_{\mathbf{P}}^{\sigma_{j}}, \Pi_{\mathbf{P}, \sigma_{j}} \phi_{\mathbf{P}}^{\sigma_{j}}\right)\right)^{2} \\
& +\int_{0}^{\infty}\left(|\mathbf{k}|-\mathbf{k} \cdot \nabla E^{\sigma_{j}}(\mathbf{P})\right) b^{\dagger}(\mathbf{k}) b(\mathbf{k}) d^{3} k+c_{\mathbf{P}}^{\sigma_{j}}
\end{aligned}
$$

where

- $\Pi_{\mathbf{P}, \sigma_{j}}:=\mathbf{P}^{p h}-g \int_{\sigma_{j}}^{\kappa} \frac{\mathbf{k}\left(b(\mathbf{k})+b^{\dagger}(\mathbf{k})\right)}{\sqrt{2}|\mathbf{k}|^{\frac{3}{2}}\left(1-\widehat{\mathbf{k}} \cdot \nabla E^{\sigma_{j}}(\mathbf{P})\right)} d^{3} k$

- $\phi_{\mathbf{P}}^{\sigma_{j}}$ (to be specified) is ground eigenvector of $H_{\mathbf{P}, \sigma_{j}}^{w}$ (note however that the "canonical" form of the Hamiltonian $H_{\mathbf{P}, \sigma_{j}}^{w}$ involves only the ray of the corresponding ground eigenvector, the same ray of $W_{\sigma_{j}}\left(\nabla E^{\sigma_{j}}(\mathbf{P})\right) \psi_{\mathbf{P}}^{\sigma_{j}}$ therefore)

- $c_{\mathbf{P}}^{\sigma_{j}}$ is an additive constant.

An iteration procedure as in Section 1 can be now carried out for the Hamiltonians $H_{\mathbf{P}, \sigma_{j}}^{w}$, acting on $F^{b}$, to construct again a sequence of related ground eigenvectors $\left\{\phi_{\mathbf{P}}^{\sigma_{j}}\right\}$. We use an analogous chain of projectors, by exploiting the spectral information known for the Hamiltonians $H_{\mathbf{P}, \sigma_{j}}$. We find that thanks to the property

$$
\phi_{\mathbf{P}}^{\sigma_{j}} \perp \Gamma_{\mathbf{P}, \sigma_{j}}^{i} \phi_{\mathbf{P}}^{\sigma_{j}}:=\left\{\Pi_{\mathbf{P}, \sigma_{j}}^{i} \phi_{\mathbf{P}}^{\sigma_{j}}-\frac{1}{\left\|\phi_{\mathbf{P}}^{\sigma_{j}}\right\|^{2}} \cdot\left(\phi_{\mathbf{P}}^{\sigma_{j}}, \Pi_{\mathbf{P}, \sigma_{j}}^{i} \phi_{\mathbf{P}}^{\sigma_{j}}\right) \phi_{\mathbf{P}}^{\sigma_{j}}\right\} \quad i=1,2,3
$$

the norm of the remainder

$$
\phi_{\mathbf{P}}^{\sigma_{j+1}}-\phi_{\mathbf{P}}^{\sigma_{j}}
$$

is of order the ratio $\frac{\sigma_{j}}{\kappa}$ raised to some positive power, in contrast to the sequence $\left\{\psi_{\mathbf{P}}^{\sigma_{j}}\right\}$. The final result is the content of Theorem 2.3 and Corollary 2.4, in which we prove the strong convergence of the sequence $\left\{\phi_{\mathbf{P}}^{\sigma_{j}}\right\}$ to a vector $\phi_{\mathbf{P}}$, in $F^{b}$. The key result is just the inequality proved, by induction, in:

Theorem 2.3 For $g$ and $\frac{\kappa}{m}$ sufficiently small, the inequality

$$
g^{2} \cdot\left|\left(\Gamma_{\mathbf{P}, \sigma_{j}}^{i} \phi_{\mathbf{P}}^{\sigma_{j}},\left(\frac{1}{H_{\mathbf{P}, \sigma_{j}}^{w}-E_{j+1}}\right) \Gamma_{\mathbf{P}, \sigma_{j}}^{i} \phi_{\mathbf{P}}^{\sigma_{j}}\right)\right|<\frac{M}{\epsilon^{\frac{j}{4}}} \quad(i=1,2,3)
$$

holds uniformly in $j$ and in $\mathbf{P} \in \Sigma$, being $M$ a sufficiently small constant. 
As straightforward consequence of the strong convergence of $\left\{\phi_{\mathbf{P}}^{\sigma_{j}}\right\}$, we obtain that the sequence $\left\{\psi_{\mathbf{P}}^{\sigma_{j}}\right\}$ goes weakly to zero in $F^{b}$, and it converges to a vector $\psi_{\mathbf{P}}$ in the representation of $\left\{b(\mathbf{k}), b^{\dagger}(\mathbf{k})\right\}$ given by the non-Fock coherent transformation $W(\nabla E(\mathbf{P}))$. Since the representations of $\left\{b(\mathbf{k}), b^{\dagger}(\mathbf{k})\right\}$, associated to the intertwiners $W(\nabla E(\mathbf{P}))$, are not equivalent for different $\mathbf{P}$, the construction of a state " $\int \psi_{\mathbf{P}} d^{3} P$ " is physically meaningless (it requires the superselection of the total momentum) [Fr.1].

In Section 3 we define a normalized ground eigenvector $\phi_{\mathbf{P}}^{\sigma}$ of $H_{\mathbf{P}, \sigma}^{w}$, where $\sigma$ lies in the continuum. The vector $\phi_{\mathbf{P}}^{\sigma}$ is strongly convergent for $\sigma \rightarrow 0$, and it is proved to carry a (strong) Hölder property with respect to $\mathbf{P}$ (in the considered neighborhood of $\mathbf{P}=0$ ), uniformly in $\sigma$, though, in general, a more regular behavior is expected $[\mathrm{Ch}]$. This is the price that we have to pay, in terms of approximation, by using regular perturbation theory.

Theorem 3.4 Under the constructive hypotheses, for $\frac{k}{m}$ and $g$ sufficiently small, the norm difference between $\phi_{\mathbf{P}}^{\sigma}$ and $\phi_{\mathbf{P}+\Delta \mathbf{P}}^{\sigma}$ is Hölder in $|\Delta \mathbf{P}|$ with coefficient $\frac{1}{16}-\delta, \delta>0$ and arbitrarily small. The multiplicative constant, $C_{\delta}$, is uniform in $0 \leq \sigma<\kappa \epsilon$, in $\mathbf{P}, \mathbf{P}+\Delta \mathbf{P} \in \Sigma$ and $\Delta \mathbf{P} \in I, I$ a sufficiently small fixed ball around $\Delta \mathbf{P}=0$.

The regularity property in $\mathbf{P}$, resulting from analytic perturbation theory, seems to be essential in the construction of the asymptotic states in the scattering theory $[\mathrm{Pi}]$.

\section{Construction of the sequence $\left\{\psi_{\mathbf{P}}^{\sigma_{j}}\right\}$}

In the present section we only construct the sequence of ground eigenvectors of the Hamiltonians $H_{\mathbf{P}, \sigma_{j}}$. In order to do it, we introduce some preliminary lemmas (1.1, $1.2,1.3)$ which are necessary to perform the projection in Theorem 1.4. Finally, in Corollary 1.5 , the sequence $\left\{\psi_{\mathbf{P}}^{\sigma_{j}}, j \in N\right\}$ is constructed by iteration.

The constructive hypotheses are:

I) the considered infrared cutoff are $\sigma_{j}=\kappa \epsilon^{\frac{j}{2}}$ where $0<\epsilon<\left(\frac{1}{5}\right)^{8}, j \in N$;

II) the momenta $\mathbf{P}$ are restricted to the set $\Sigma \equiv\left\{\mathbf{P}:|\mathbf{P}| \leq \frac{m}{20}\right\}$;

III) the ratio $\frac{\kappa}{m}$ fulfills the inequality: $\frac{\kappa}{m}\left(2 \pi g^{2}+\frac{3}{5} \epsilon^{\frac{1}{2}}\right) \leq \frac{1}{200}$.

We synthesize the content of the three lemmas:

- in Lemma 1.1, starting from $\left.H_{\mathbf{P}, \sigma_{j}}\right|_{F_{\sigma_{j}}^{+}}$, we study the operator $H_{\mathbf{P}, \sigma_{j}}$ applied to the subspace $F_{\sigma_{j+1}}^{+}$and we show how to recover the new eigenvector and the new gap for the same operator $\left(H_{\mathbf{P}, \sigma_{j}}\right)$ on the larger space $F_{\sigma_{j+1}}^{+}$, which however does not contain new interacting bosons compared with $F_{\sigma_{j}}^{+}$;

- in Lemma 1.2 the ground energy is checked to be not decreasing in the infrared cutoff:

$$
E_{\mathbf{P}}^{\sigma_{j}} \geq E_{\mathbf{P}}^{\sigma_{j+1}}
$$


- in Lemma 1.3 the meaning of the "smallness" of

$$
\left(\Delta H_{\mathbf{P}}\right)_{\sigma_{j+1}}^{\sigma_{j}}=H_{\mathbf{P}, \sigma_{j+1}}-H_{\mathbf{P}, \sigma_{j}}
$$

with respect to $\left.H_{\mathbf{P}, \sigma_{j}}\right|_{F_{\sigma_{j+1}}^{+}}$is explained.

Remark. The value of $g(g>0)$ will be constrained several times during the procedure; at each time we call $g$ the maximum value such that the constraint under examination as well as the previous constraints are satisfied. In Sections 2, 3 we assume a sufficiently small ratio $\frac{\kappa}{m}$ to prove the convergence of the transformed sequence $\left\{\phi_{\mathbf{P}}^{\sigma_{j}}\right\}$ and the regularity properties. The proofs of Section 1 do not require the restrictive constraint on $\epsilon$ but only $0<\epsilon<1$ and moreover can be extended to arbitrary (finite) values of $\frac{k}{m}$ if one assumes some results by Fröhlich [Fr.2] concerning the ground energy.

Lemma 1.1 If $\psi_{\mathbf{P}}^{\sigma_{j}}$ is the unique ground eigenvector of $\left.H_{\mathbf{P}, \sigma_{j}}\right|_{F_{\sigma_{j}}^{+}}$with corresponding gap larger than $\frac{\sigma_{j}}{2}$, then $\psi_{\mathbf{P}}^{\sigma_{j}} \otimes \psi_{0}, \psi_{0}$ vacuum state in $F_{\sigma_{j+1}}^{\sigma_{j}}$ (defined below), is the unique ground eigenvector of $\left.H_{\mathbf{P}, \sigma_{j}}\right|_{F_{\sigma_{j+1}}^{+}}$with the same eigenvalue $E_{\mathbf{P}}^{\sigma_{j}}$ (of $\left.\psi_{\mathbf{P}}^{\sigma_{j}}\right)$ and its gap is larger than $\frac{3}{5} \sigma_{j+1}$.

Proof ${ }^{2}$. Let us decompose $F_{\sigma_{j+1}}^{+}$as $F_{\sigma_{j}}^{+} \otimes F_{\sigma_{j+1}}^{\sigma_{j}}$, where $F_{\sigma_{j+1}}^{\sigma_{j}}$ is the tensorial subproduct defined as follows

$$
F_{\sigma_{j+1}}^{\sigma_{j}} \equiv F(h), \quad h:=L^{2}\left(B_{\sigma_{j+1}}^{\sigma_{j}}, d^{3} k\right), B_{\sigma_{j+1}}^{\sigma_{j}}:=\left\{\mathbf{k}: \sigma_{j+1}<|\mathbf{k}|<\sigma_{j}\right\} .
$$

Clearly the vector $\psi_{\mathbf{P}}^{\sigma_{j}} \otimes \psi_{0}$ is an eigenvector of $\left.H_{\mathbf{P}, \sigma_{j}}\right|_{F_{\sigma_{j+1}}^{+}}$, with eigenvalue $E_{\mathbf{P}}^{\sigma_{j}}$, and

$$
H_{\mathbf{P}, \sigma_{j}}: F_{\sigma_{j+1}}^{+} \ominus\left\{\psi_{\mathbf{P}}^{\sigma_{j}} \otimes \psi_{0}\right\} \rightarrow F_{\sigma_{j+1}}^{+} \ominus\left\{\psi_{\mathbf{P}}^{\sigma_{j}} \otimes \psi_{0}\right\}
$$

where $\left\{\psi_{\mathbf{P}}^{\sigma_{j}} \otimes \psi_{0}\right\}$ denotes the subspace generated by $\psi_{\mathbf{P}}^{\sigma_{j}} \otimes \psi_{0}$.

For this reason the gap we want to estimate can be analyzed starting from

$$
\left.\inf _{\text {spec }}\left\{\left.H_{\mathbf{P}, \sigma_{j}}\right|_{F_{\sigma_{j+1}}^{+} \ominus\left\{\psi_{\mathbf{P}}^{\sigma_{j}} \otimes \psi_{0}\right.}\right\}\right\}
$$

if the above quantity is larger than $E_{\mathbf{P}}^{\sigma_{j}}$. In this case the gap corresponds to

$$
\inf _{\text {spec }}\left\{\left.H_{\mathbf{P}, \sigma_{j}}\right|_{F_{\sigma_{j+1}}^{+} \ominus\left\{\psi_{\mathbf{P}}^{\sigma_{j}} \otimes \psi_{0}\right\}}-E_{\mathbf{P}}^{\sigma_{j}}\right\} \text {. }
$$

Since it is useful in Lemma 1.3, we prove a stronger result:

$$
\inf _{\text {spec }}\left\{\left.H_{\mathbf{P}, \sigma_{j}}\right|_{F_{\sigma_{j+1}}^{+} \ominus\left\{\psi_{\mathbf{P}}^{\sigma_{j}} \otimes \psi_{0}\right\}}-\left.\frac{1}{5} H^{p h}\right|_{\sigma_{j+1}} ^{\sigma_{j}}-E_{\mathbf{P}}^{\sigma_{j}}\right\} \geq \frac{3}{5} \sigma_{j+1}
$$

\footnotetext{
${ }^{2}$ I am indebted to J. Fröhlich for having suggested to me a shorter proof and for a helpful discussion of the lemma.
} 
having defined

$$
\left.H^{p h}\right|_{\sigma_{j+1}} ^{\sigma_{j}}:=\int_{\sigma_{j+1}}^{\sigma_{j}}|\mathbf{k}| b^{\dagger}(\mathbf{k}) b(\mathbf{k}) d^{3} k \quad \text { and }\left.\quad N\right|_{\sigma_{j+1}} ^{\sigma_{j}}:=\int_{\sigma_{j+1}}^{\sigma_{j}} b^{\dagger}(\mathbf{k}) b(\mathbf{k}) d^{3} k .
$$

For this purpose note that $\left[H_{\mathbf{P}, \sigma_{j}}, n(\mathbf{k})\right]=0$ for $|\mathbf{k}|<\sigma_{j}$, where $n(\mathbf{k}):=$ $b^{\dagger}(\mathbf{k}) b(\mathbf{k})$, in distributional sense; it implies that our search of the infimum can be restricted to the analysis of the expectation value of

$$
\left.H_{\mathbf{P}, \sigma_{j}}\right|_{F_{\sigma_{j+1}}^{+} \ominus\left\{\psi_{\mathbf{P}}^{\sigma_{j}} \otimes \psi_{0}\right\}}-\left.\frac{1}{5} H^{p h}\right|_{\sigma_{j+1}} ^{\sigma_{j}}
$$

on vectors like $\varphi \otimes \eta, \varphi \otimes \eta \perp \psi_{\mathbf{P}}^{\sigma_{j}} \otimes \psi_{0}$ and $\|\varphi\|=\|\eta\|=1$, where $\varphi \in F_{\sigma_{j}}^{+}$is in the domain of $H_{\mathbf{P}, \sigma_{j}}, \eta \in F_{\sigma_{j+1}}^{\sigma_{j}}$ is in the domain of $\left.H^{p h}\right|_{\sigma_{j+1}} ^{\sigma_{j}}$ and it is eigenvector of $N||_{\sigma_{j+1}}^{\sigma_{j}}$.

It is sufficient to distinguish two different energy regimes, corresponding to vectors $\eta$ with spectral support, in $\left.H^{p h}\right|_{\sigma_{j+1}} ^{\sigma_{j}}$, below and above the value $\frac{m}{20}$ respectively; let us define

$$
q:=\left(\eta,\left.H^{p h}\right|_{\sigma_{j+1}} ^{\sigma_{j}} \eta\right)
$$

then in the first case $q \leq \frac{m}{20}$, in the second one $q>\frac{m}{20}$.

1) $q \leq \frac{m}{20}$

For the set $\left\{\mathbf{P}^{\prime}:\left|\mathbf{P}^{\prime}\right| \leq|\mathbf{P}|+q \quad, \mathbf{P} \in \Sigma\right\}$ the condition on $q$ implies $\left|\nabla E^{\sigma_{j}}\left(\mathbf{P}^{\prime}\right)\right|<$ $\frac{1}{5}$, by steps as in Lemma A2, Appendix. Moreover we can estimate

$$
\left(\varphi \otimes \eta,\left\{H_{\mathbf{P}, \sigma_{j}}-\left.\frac{1}{5} H^{p h}\right|_{\sigma_{j+1}} ^{\sigma_{j}}-E_{\mathbf{P}}^{\sigma_{j}}\right\} \varphi \otimes \eta\right)
$$

from below in terms of

$$
\min \left\{\frac{1}{2} \sigma_{j}, \inf _{\mathbf{q}: \frac{m}{20} \geq|\mathbf{q}| \geq \sigma_{j+1}}\left\{E_{\mathbf{P}-\mathbf{q}}^{\sigma_{j}}-E_{\mathbf{P}}^{\sigma_{j}}+\frac{4}{5}|\mathbf{q}|\right\}\right\}
$$

due to the following facts:

- the gap of $\left.H_{\mathbf{P}, \sigma_{j}}\right|_{F_{\sigma_{j}}^{+}}$is bigger than $\frac{\sigma_{j}}{2}$, by hypothesis;

- the inequality which holds for $\eta \perp \psi_{0}$

$$
\begin{aligned}
& \left(\varphi \otimes \eta,\left\{H_{\mathbf{P}, \sigma_{j}}-\left.\frac{1}{5} H^{p h}\right|_{\sigma_{j+1}} ^{\sigma_{j}}-E_{\mathbf{P}}^{\sigma_{j}}\right\} \varphi \otimes \eta\right) \\
& \geq \inf _{\mathbf{q}: \frac{m}{20} \geq|\mathbf{q}| \geq \sigma_{j+1}}\left(\varphi,\left\{\frac{\left(\mathbf{P}^{p h}-\mathbf{P}+\mathbf{q}\right)^{2}}{2 m}\right.\right. \\
& \left.\left.\quad+g \int_{\sigma_{j}}^{\kappa} \frac{b(\mathbf{k})+b^{\dagger}(\mathbf{k})}{\sqrt{2|\mathbf{k}|}} d^{3} k+\left.H^{p h}\right|_{\sigma_{j}} ^{+\infty}+\frac{4}{5}|\mathbf{q}|-E_{\mathbf{P}}^{\sigma_{j}}\right\} \varphi\right) \\
& \geq_{\mathbf{q}: \frac{m}{20} \geq|\mathbf{q}| \geq \sigma_{j+1}}\left\{E_{\mathbf{P}-\mathbf{q}}^{\sigma_{j}}-E_{\mathbf{P}}^{\sigma_{j}}+\frac{4}{5}|\mathbf{q}|\right\} .
\end{aligned}
$$


From the constraint on the gradient and being $\frac{1}{2} \sigma_{j} \geq \frac{3}{5} \sigma_{j+1}$, we can conclude that

$$
\min \left\{\frac{1}{2} \sigma_{j}, \inf _{\mathbf{q}: \frac{m}{20} \geq|\mathbf{q}| \geq \sigma_{j+1}}\left\{E_{\mathbf{P}-\mathbf{q}}^{\sigma_{j}}-E_{\mathbf{P}}^{\sigma_{j}}+\frac{4}{5}|\mathbf{q}|\right\}\right\} \geq \frac{3}{5} \sigma_{j+1} .
$$

2) $q>\frac{m}{20}$

Let us start observing that

$$
\left.H_{\mathbf{P}, \sigma_{j}}\right|_{F_{\sigma_{j+1}}^{+}}-\left.H^{p h}\right|_{\sigma_{j+1}} ^{\sigma_{j}}+2 \pi g^{2} \kappa \geq 0
$$

to provide the bound

$$
\begin{aligned}
\left(\varphi \otimes \eta,\left\{H_{\mathbf{P}, \sigma_{j}}-\left.\frac{1}{5} H^{p h}\right|_{\sigma_{j+1}} ^{\sigma_{j}}-E_{\mathbf{P}}^{\sigma_{j}}\right\} \varphi \otimes \eta\right) & \geq \frac{4}{5} q-2 \pi g^{2} \kappa-E_{\mathbf{P}}^{\sigma_{j}} \\
& \geq \frac{m}{25}-2 \pi g^{2} \kappa-E_{\mathbf{P}}^{\sigma_{j}} .
\end{aligned}
$$

Now, from the constraints on the ratio $\frac{\kappa}{m}$, on $\Sigma$, and from next Lemma 1.2 which guarantees $E_{\mathbf{P}}^{\sigma_{0}} \geq E_{\mathbf{P}}^{\sigma_{j}}$, we have

$$
\frac{m}{25}-2 \pi g^{2} \kappa-E_{\mathbf{P}}^{\sigma_{j}} \geq \frac{m}{25}-2 \pi g^{2} \kappa-E_{\mathbf{P}}^{\sigma_{0}} \geq \frac{m}{25}-2 \pi g^{2} \kappa-\frac{m}{2 \cdot 20^{2}} \geq \frac{3}{5} \sigma_{1} \geq \frac{3}{5} \sigma_{j+1} .
$$

where the inequality

$$
\frac{m}{25}-2 \pi g^{2} \kappa-\frac{m}{2 \cdot 20^{2}} \geq \frac{3}{5} \sigma_{1}
$$

derives from the assumption

$$
2 \pi g^{2} \kappa+\frac{3}{5} \kappa \epsilon^{\frac{1}{2}} \leq \frac{m}{200} .
$$

Conclusion

$$
\inf _{\text {spec }}\left\{\left.H_{\mathbf{P}, \sigma_{j}}\right|_{F_{\sigma_{j+1}}^{+} \ominus\left\{\psi_{\mathbf{P}}^{\sigma_{j}} \otimes \psi_{0}\right\}}-\left.\frac{1}{5} H^{p h}\right|_{\sigma_{j+1}} ^{\sigma_{j}}-E_{\mathbf{P}}^{\sigma_{j}}\right\} \geq \frac{3}{5} \sigma_{j+1} .
$$

Lemma 1.2 The following relation between $E_{\mathbf{P}}^{\sigma_{j}}$ and $E_{\mathbf{P}}^{\sigma_{j+1}}$ (ground energy of $\left.\left.H_{\mathbf{P}, \sigma_{j+1}}\right|_{F_{\sigma_{j+1}}^{+}}\right)$holds:

$$
E_{\mathbf{P}}^{\sigma_{j}} \geq E_{\mathbf{P}}^{\sigma_{j+1}} \geq E_{\mathbf{P}}^{\sigma_{j}}-10 \pi g^{2} \sigma_{j}
$$

Proof. Considering that

$$
\left.H_{\mathbf{P}, \sigma_{j+1}}\right|_{F_{\sigma_{j+1}}^{+}}=\left.H_{\mathbf{P}, \sigma_{j}}\right|_{F_{\sigma_{j+1}}^{+}}+I_{F_{\sigma_{j}}^{+}} \otimes g \int_{\sigma_{j+1}}^{\sigma_{j}}\left(b(\mathbf{k})+b^{\dagger}(\mathbf{k})\right) \frac{d^{3} \mathbf{k}}{\sqrt{2}|\mathbf{k}|^{\frac{1}{2}}},
$$


$\left(I_{F_{\sigma_{j}}^{+}}\right.$is the identity operator in the space $F_{\sigma_{j}}^{+}$) the expectation value of $H_{\mathbf{P}, \sigma_{j+1}}$ on $\psi_{\mathbf{P}}^{\sigma_{j}} \otimes \psi_{0}$

$$
\frac{\left(\psi_{\mathbf{P}}^{\sigma_{j}} \otimes \psi_{0}, H_{\mathbf{P}, \sigma_{j+1}} \psi_{\mathbf{P}}^{\sigma_{j}} \otimes \psi_{0}\right)}{\left\|\psi_{\mathbf{P}}^{\sigma_{j}} \otimes \psi_{0}\right\|^{2}}
$$

coincides with $E_{\mathbf{P}}^{\sigma_{j}}$.

$E_{\mathbf{P}}^{\sigma_{j+1}}$ is the infimum of the expectation value of $H_{\mathbf{P}, \sigma_{j+1}}$ on the vectors, in $F_{\sigma_{j+1}}^{+}$, belonging to the operator domain, by definition. Therefore $E_{\mathbf{P}}^{\sigma_{j+1}} \leq E_{\mathbf{P}}^{\sigma_{j}}$ and, in general, $E_{\mathbf{P}}^{\sigma^{\prime \prime}} \leq E_{\mathbf{P}}^{\sigma^{\prime}}$ for $\sigma^{\prime} \geq \sigma^{\prime \prime}$. Moreover collecting the following results

- as proved in the previous lemma

$$
\inf _{\text {spec }}\left(\left.H_{\mathbf{P}, \sigma_{j}}\right|_{F_{\sigma_{j+1}}^{+}}-\left.\frac{1}{5} H^{p h}\right|_{\sigma_{j+1}} ^{\sigma_{j}}\right) \geq E_{\mathbf{P}}^{\sigma_{j}}
$$

- being

$$
\left.\frac{1}{5} H^{p h}\right|_{\sigma_{j+1}} ^{\sigma_{j}}+g \int_{\sigma_{j+1}}^{\sigma_{j}}\left(b(\mathbf{k})+b^{\dagger}(\mathbf{k})\right) \frac{d^{3} k}{\sqrt{2}|\mathbf{k}|^{\frac{1}{2}}}+10 \pi g^{2}\left(\sigma_{j}-\sigma_{j+1}\right) \geq 0
$$

we can estimate

$$
\begin{aligned}
E_{\mathbf{P}}^{\sigma_{j+1}} & =\inf _{\text {spec }}\left(\left.H_{\mathbf{P}, \sigma_{j}}\right|_{F_{\sigma_{j+1}}^{+}}+g \int_{\sigma_{j+1}}^{\sigma_{j}}\left(b(\mathbf{k})+b^{\dagger}(\mathbf{k})\right) \frac{d^{3} k}{\sqrt{2}|\mathbf{k}|^{\frac{1}{2}}}\right) \\
& \geq \inf _{\text {spec }}\left(\left.H_{\mathbf{P}, \sigma_{j}}\right|_{F_{\sigma_{j+1}}^{+}}-\left.\frac{1}{5} H^{p h}\right|_{\sigma_{j+1}} ^{\sigma_{j}}-10 \pi g^{2} \sigma_{j}\right) \\
& \geq E_{\mathbf{P}}^{\sigma_{j}}-10 \pi g^{2} \sigma_{j}
\end{aligned}
$$

Lemma 1.3 For properly small values of $g$ and under the hypotheses of Lemma 1.1, $\left(\Delta H_{\mathbf{P}}\right)_{\sigma_{j+1}}^{\sigma_{j}}=g \int_{\sigma_{j+1}}^{\sigma_{j}}\left(b(\mathbf{k})+b^{\dagger}(\mathbf{k})\right) \frac{d^{3} \mathbf{k}}{\sqrt{2}|\mathbf{k}|}$ is small with respect to $\left.H_{\mathbf{P}, \sigma_{j}}\right|_{F_{\sigma_{j+1}}^{+}}$in the following sense: For $E_{j+1} \in \mathcal{C}$ such that

$$
\left|E_{j+1}-\inf _{\text {spec }}\left(\left.H_{\mathbf{P}, \sigma_{j}}\right|_{F_{\sigma_{j+1}}^{+}}\right)\right|=\left|E_{j+1}-E_{\mathbf{P}}^{\sigma_{j}}\right|=\frac{11}{20} \sigma_{j+1},
$$

it turns out that

$$
\left\|\frac{1}{H_{\mathbf{P}, \sigma_{j}}-E_{j+1}}\left(-\left(\Delta H_{\mathbf{P}}\right)_{\sigma_{j+1}}^{\sigma_{j}} \frac{1}{H_{\mathbf{P}, \sigma_{j}}-E_{j+1}}\right)^{n}\right\|_{F_{\sigma_{j+1}}^{+}} \leq \frac{20\left(C_{g}\right)^{n}}{\sigma_{j+1}}
$$

where $C_{g}, 0<C_{g}<\frac{1}{12}$, is a constant independent of $j$.

It implies the validity of the series expansion:

$$
\begin{aligned}
& \left.\frac{1}{H_{\mathbf{P}, \sigma_{j}}+\left(\Delta H_{\mathbf{P}}\right)_{\sigma_{j+1}}^{\sigma_{j}}-E_{j+1}}\right|_{F_{\sigma_{j+1}}^{+}} \\
& \quad=\left.\frac{1}{H_{\mathbf{P}, \sigma_{j}}-E_{j+1}} \sum_{n=0}^{+\infty}\left(-\left(\Delta H_{\mathbf{P}}\right)_{\sigma_{j+1}}^{\sigma_{j}} \frac{1}{H_{\mathbf{P}, \sigma_{j}}-E_{j+1}}\right)^{n}\right|_{F_{\sigma_{j+1}}^{+}} .
\end{aligned}
$$


Proof. The aim of this lemma is to arrive at a norm controlled series expansion of the resolvent, to be exploited in Theorem 1.4.

Let us analyze the $n^{\text {th }}$ term of the series

$$
\left.\frac{1}{H_{\mathbf{P}, \sigma_{j}}-E_{j+1}} \sum_{n=0}^{+\infty}\left(-\left(\Delta H_{\mathbf{P}}\right)_{\sigma_{j+1}}^{\sigma_{j}} \frac{1}{H_{\mathbf{P}, \sigma_{j}}-E_{j+1}}\right)^{n}\right|_{F_{\sigma_{j+1}}^{+}}
$$

namely

$$
(-1)^{n}\left(\frac{1}{H_{\mathbf{P}, \sigma_{j}}-E_{j+1}}\right)^{\frac{1}{2}} \cdots\left(\frac{1}{H_{\mathbf{P}, \sigma_{j}}-E_{j+1}}\right)^{\frac{1}{2}}\left(\Delta H_{\mathbf{P}}\right)_{\sigma_{j+1}}^{\sigma_{j}}\left(\frac{1}{H_{\mathbf{P}, \sigma_{j}}-E_{j+1}}\right)^{\frac{1}{2}} \cdots\left(\frac{1}{H_{\mathbf{P}, \sigma_{j}}-E_{j+1}}\right)^{\frac{1}{2}}
$$

where

$$
\left(\frac{1}{H_{\mathbf{P}, \sigma_{j}}-E_{j+1}}\right)^{\frac{1}{2}}
$$

is defined starting from the spectral representation of $H_{\mathbf{P}, \sigma_{j}}$, by using the convention to take the branch of the square root with smaller argument in $(-\pi, \pi]$. Each term in the series expansion is under control if we are able to estimate the norm:

$$
\left\|\left(\frac{1}{H_{\mathbf{P}, \sigma_{j}}-E_{j+1}}\right)^{\frac{1}{2}}\left(\Delta H_{\mathbf{P}}\right)_{\sigma_{j+1}}^{\sigma_{j}}\left(\frac{1}{H_{\mathbf{P}, \sigma_{j}}-E_{j+1}}\right)^{\frac{1}{2}}\right\|_{F_{\sigma_{j+1}^{+}}} .
$$

This norm is less or equal to

$$
2 g\left\|\left(\frac{1}{H_{\mathbf{P}, \sigma_{j}}-E_{j+1}}\right)^{\frac{1}{2}}\right\|_{F_{\sigma_{j+1}^{+}}^{+}} \cdot\left\|\left(\int_{\sigma_{j+1}}^{\sigma_{j}} \frac{b(\mathbf{k})}{\sqrt{2}|\mathbf{k}|^{\frac{1}{2}}} d^{3} k\right)\left(\frac{1}{H_{\mathbf{P}, \sigma_{j}}-E_{j+1}}\right)^{\frac{1}{2}}\right\|_{F_{\sigma_{j+1}^{+}}^{+}}
$$

if the quantities written above exist.

Moreover the following estimate holds:

$$
\left\|\int_{\sigma_{j+1}}^{\sigma_{j}} b(\mathbf{k}) \frac{d^{3} k}{\sqrt{2|\mathbf{k}|}}\left(\frac{1}{H_{\mathbf{P}, \sigma_{j}}-E_{j+1}}\right)^{\frac{1}{2}}\right\|_{F_{\sigma_{j+1}}^{+}} \leq \sqrt{10 \pi} \cdot \sigma_{j}^{\frac{1}{2}} .
$$

Indeed, starting from vectors belonging to $D^{b} \bigcap F_{\sigma_{j+1}}^{+}\left(D^{b}:=\bigvee_{n \in N} \Phi_{\mathbf{P}}^{n}\right.$, see point iv) in Definition of the model), we get the bound:

$$
\begin{aligned}
& \left\|\int_{\sigma_{j+1}}^{\sigma_{j}} b(\mathbf{k}) \frac{d^{3} k}{\sqrt{2|\mathbf{k}|}}\left(\frac{1}{H_{\mathbf{P}, \sigma_{j}}-E_{j+1}}\right)^{\frac{1}{2}}\right\|_{F_{\sigma_{j+1}^{+}}^{+}}^{2} \\
& \quad \leq 2 \pi \sigma_{j} \cdot\left\|\left.H^{p h}\right|_{\sigma_{j+1}} ^{\sigma_{j}}\left[\left(\frac{1}{H_{\mathbf{P}, \sigma_{j}}-E_{j+1}}\right)^{\frac{1}{2}}\right]^{\dagger}\left(\frac{1}{H_{\mathbf{P}, \sigma_{j}}-E_{j+1}}\right)^{\frac{1}{2}}\right\|_{F_{\sigma_{j+1}}}
\end{aligned}
$$

in this respect note that $\left[H_{\mathbf{P}, \sigma_{j}},\left.H^{p h}\right|_{\sigma_{j+1}} ^{\sigma_{j}}\right]=0$. 
The operator norm of

$$
\left.\left.H^{p h}\right|_{\sigma_{j+1}} ^{\sigma_{j}}\left[\left(\frac{1}{H_{\mathbf{P}, \sigma_{j}}-E_{j+1}}\right)^{\frac{1}{2}}\right]^{\dagger}\left(\frac{1}{H_{\mathbf{P}, \sigma_{j}}-E_{j+1}}\right)^{\frac{1}{2}}\right|_{F_{\sigma_{j+1}}^{+}}
$$

can be studied on $\psi_{\mathbf{P}}^{\sigma_{j}} \otimes \psi_{0}$ and on $F_{\sigma_{j+1}}^{+} \ominus\left\{\psi_{\mathbf{P}}^{\sigma_{j}} \otimes \psi_{0}\right\}$ separately. The operator vanishes on $\psi_{\mathbf{P}}^{\sigma_{j}} \otimes \psi_{0}$ (put $\left.H^{p h}\right|_{\sigma_{j+1}} ^{\sigma_{j}}$ on the right). The discussion is then restricted to the subspace $F_{\sigma_{j+1}}^{+} \ominus\left\{\psi_{\mathbf{P}}^{\sigma_{j}} \otimes \psi_{0}\right\}$.

As already seen in Lemma 1.1, we have

$$
\inf _{\text {spec }}\left(\left.H_{\mathbf{P}, \sigma_{j}}\right|_{F_{\sigma_{j+1}}^{+} \ominus\left\{\psi_{\mathbf{P}}^{\sigma_{j}} \otimes \psi_{0}\right\}}-\left.\frac{1}{5} H^{p h}\right|_{\sigma_{j+1}} ^{\sigma_{j}}\right) \geq E_{\mathbf{P}}^{\sigma_{j}}+\frac{3}{5} \sigma_{j+1}
$$

from which

$$
\inf _{\text {spec }}\left(\left.H_{\mathbf{P}, \sigma_{j}}\right|_{F_{\sigma_{j+1}}^{+} \ominus\left\{\psi_{\mathbf{P}}^{\sigma_{j}} \otimes \psi_{0}\right\}}-\left.\frac{1}{5} H^{p h}\right|_{\sigma_{j+1}} ^{\sigma_{j}}-R e E_{j+1}\right) \geq \frac{3}{5} \sigma_{j+1}-\frac{11}{20} \sigma_{j+1}>0 .
$$

Going to the joint spectral representation of the operators $H_{\mathbf{P}, \sigma_{j}}$ and $\left.H^{p h}\right|_{\sigma_{j+1}} ^{\sigma_{j}}$, we easily obtain

$$
\left\|\left.H^{p h}\right|_{\sigma_{j+1}} ^{\sigma_{j}}\left[\left(\frac{1}{H_{\mathbf{P}, \sigma_{j}}-E_{j+1}}\right)^{\frac{1}{2}}\right]^{\dagger}\left(\frac{1}{H_{\mathbf{P}, \sigma_{j}}-E_{j+1}}\right)^{\frac{1}{2}}\right\|_{F_{\sigma_{j+1}^{+}}} \leq 5 .
$$

\section{Conclusion}

For $g$ sufficiently small but uniform in $j$, the thesis is proved because the norm

$$
\left\|\left(\frac{1}{H_{\mathbf{P}, \sigma_{j}}-E_{j+1}}\right)^{\frac{1}{2}}\right\|_{F_{\sigma_{j+1}^{+}}}
$$

is of order $\left(\frac{1}{\sigma_{j+1}}\right)^{\frac{1}{2}}$.

Now, on the basis of the previous results and starting from the relation between the resolvents of the Hamiltonians $H_{\mathbf{P}, \sigma_{j+1}}$ and $H_{\mathbf{P}, \sigma_{j}}$ applied on the subspace $F_{\sigma_{j+1}}^{+}$, we can construct $\psi_{\mathbf{P}}^{\sigma_{j+1}}$ and establish that the norm difference between the ground eigenvectors, $\psi_{\mathbf{P}}^{\sigma_{j+1}}$ and $\psi_{\mathbf{P}}^{\sigma_{j}}$, is bounded by a constant strictly less than 1. Concerning notations, starting from now, we identify $\psi_{\mathbf{P}}^{\sigma_{j}}$ and $\psi_{\mathbf{P}}^{\sigma_{j}} \otimes \psi_{0}$ as vectors in $F_{\sigma_{j+1}}^{+}$.

Theorem 1.4 Under the constructive hypotheses and for $g$ sufficiently small, if $\psi_{\mathbf{P}}^{\sigma_{j}}$ is the unique ground eigenvector of $\left.H_{\mathbf{P}, \sigma_{j}}\right|_{F_{\sigma_{j}}^{+}}$with gap larger than $\frac{\sigma_{j}}{2}$, $\left.H_{\mathbf{P}, \sigma_{j+1}}\right|_{F_{\sigma_{j+1}}^{+}}$has a unique ground eigenvector $\psi_{\mathbf{P}}^{\sigma_{j+1}}$ of energy $E_{\mathbf{P}}^{\sigma_{j+1}}$ and the 
corresponding gap is larger than $\frac{\sigma_{j+1}}{2}$; the (unnormalized) vector $\psi_{\mathbf{P}}^{\sigma_{j+1}}$ is so defined

$$
\psi_{\mathbf{P}}^{\sigma_{j+1}}:=-\frac{1}{2 \pi i} \oint \frac{1}{H_{\mathbf{P}, \sigma_{j+1}}-E_{j+1}} d E_{j+1} \psi_{\mathbf{P}}^{\sigma_{j}}
$$

where $E_{j+1} \in \mathcal{C}$ and $\left|E_{j+1}-E_{\mathbf{P}}^{\sigma_{j}}\right|=\frac{11}{20} \sigma_{j+1}$.

Proof. Continuity argument.

We consider the series expansion of the resolvent (inside the integral (3)) which is provided by Lemma 1.3. We distinguish the coupling constant $g$ in $\left.H_{\mathbf{P}, \sigma_{j}}\right|_{F_{\sigma_{j+1}}^{+}}$ from that one in $\left(\Delta H_{\mathbf{P}}\right)_{\sigma_{j+1}}^{\sigma_{j}}$, and we denote the latter by $g^{\sharp}$. Kato-Rellich theorem ensures that (3) is well defined for sufficiently small $g^{\sharp}$, since the gap of $\left.H_{\mathbf{P}, \sigma_{j}}\right|_{F_{\sigma_{j+1}}^{+}}$ is bigger or equal to $\frac{3}{5} \sigma_{j+1}$ (Lemma 1.1) and $\left(\Delta H_{\mathbf{P}}\left(g^{\sharp}\right)\right)_{\sigma_{j+1}}^{\sigma_{j}}$ is a small Kato perturbation with respect to $\left.H_{\mathbf{P}, \sigma_{j}}\right|_{F_{\sigma_{j+1}}^{+}}$. Now look at the figure,

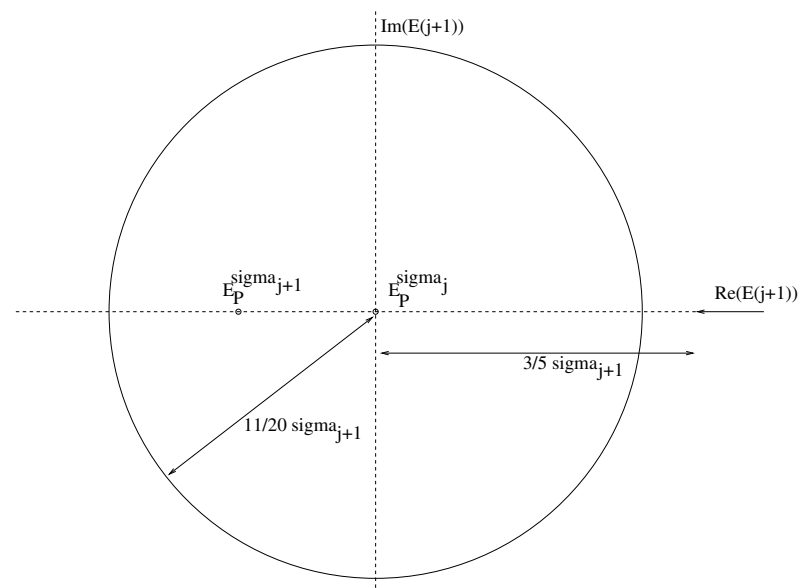

as $g^{\sharp}$ increases, the definition (3) is surely consistent as long as the perturbed eigenvalue $E_{\mathbf{P}}^{\sigma_{j+1}}\left(g^{\sharp}\right)$ remains inside the circle of integration and the remaining spectrum of $\left.H_{\mathbf{P}, \sigma_{j+1}}\left(g^{\sharp}\right)\right|_{F_{\sigma_{j+1}}^{+}}:=\left.H_{\mathbf{P}, \sigma_{j}}\right|_{F_{\sigma_{j+1}}^{+}}+\left(\Delta H_{\mathbf{P}}\left(g^{\sharp}\right)\right)_{\sigma_{j+1}}^{\sigma_{j}}$ is localized outside.

According to the estimates given in Lemma 1.3, we can conclude that:

i) the integral

$$
-\frac{1}{2 \pi i} \oint\left\|\frac{1}{H_{\mathbf{P}, \sigma_{j+1}}\left(g^{\sharp}\right)-E_{j+1}} \psi_{\mathbf{P}}^{\sigma_{j}}\right\|_{F_{\sigma_{j+1}}^{+}} d E_{j+1}
$$

exists for $0 \leq g^{\sharp} \leq g$; 
ii) the vector $\psi_{\mathbf{P}}^{\sigma_{j+1}}$ is not zero since

$$
\begin{aligned}
& \psi_{\mathbf{P}}^{\sigma_{j+1}} \\
& =\psi_{\mathbf{P}}^{\sigma_{j}}-\frac{1}{2 \pi i} \sum_{n=1}^{+\infty} \oint \frac{1}{H_{\mathbf{P}, \sigma_{j}}-E_{j+1}}\left(-\left(\Delta H_{\mathbf{P}}\right)_{\sigma_{j+1}}^{\sigma_{j}} \frac{1}{H_{\mathbf{P}, \sigma_{j}}-E_{j+1}}\right)^{n} d E_{j+1} \psi_{\mathbf{P}}^{\sigma_{j}}
\end{aligned}
$$

and the norm of the remainder, $\psi_{\mathbf{P}}^{\sigma_{j+1}}-\psi_{\mathbf{P}}^{\sigma_{j}}$, is less than $\frac{11 C_{g}}{1-C_{g}} \cdot\left\|\psi_{\mathbf{P}}^{\sigma_{j}}\right\|$, therefore

$$
\left\|\psi_{\mathbf{P}}^{\sigma_{j+1}}\right\| \geq \frac{1-12 C_{g}}{1-C_{g}} \cdot\left\|\psi_{\mathbf{P}}^{\sigma_{j}}\right\|>0
$$

iii) the ground state of $\left.H_{\mathbf{P}, \sigma_{j+1}}\right|_{F_{\sigma_{j+1}}^{+}}$is unique by continuity;

iv) since $E_{\mathbf{P}}^{\sigma_{j+1}} \leq E_{\mathbf{P}}^{\sigma_{j}}$ (Lemma 1.2), the new gap is larger than $\frac{\sigma_{j+1}}{2}$.

Corollary 1.5 The sequence $\left\{\psi_{\mathbf{P}}^{\sigma_{j}}, j \in N\right\}$ is well defined.

Proof. Thanks to the results of Lemma 1.1, 1.2, 1.3 and Theorem 1.4, it is possible to iterate the procedure at fixed $g$, starting from the vacuum state (of $F^{b}$ ) $\psi_{0}$ at the level $j=0$. The iteration is consistent and does not stop since the vector obtained at the step $j+1$ has norm larger than a fixed fraction of the norm of the vector at the $j$ step. At each step the infrared cutoff is reduced by a factor $\epsilon^{\frac{1}{2}}$.

\section{Convergence of the ground eigenvectors of the transformed Hamiltonians}

As we know from [Fr.1], the Hamiltonians $H_{\mathbf{P}}$ have a ground state for representations of $\left\{b(\mathbf{k}), b^{\dagger}(\mathbf{k})\right\}$ which are coherent and non-Fock due to their infrared behavior $(\mathbf{k} \rightarrow 0)$, so that the sequence $\left\{\psi_{\mathbf{P}}^{\sigma_{j}}\right\}$ cannot converge in $F^{b}$ to a nonzero vector. The correct coherent factor can be re-obtained by a heuristic argument which singles out the expected infrared limit of the coherent factor, explicitly in the case $\mathbf{P}=0$ and implicitly in the case $\mathbf{P} \neq 0, \mathbf{P} \in \Sigma$. Such an information is then used in a rigorous proof which is based on the iterative procedure of construction of the ground state. In particular, for each infrared cutoff $\sigma_{j}$, we consider a Hamiltonian $H_{\mathbf{P}, \sigma_{j}}^{w}$ unitarily equivalent to $H_{\mathbf{P}, \sigma_{j}}$, according to a coherent transformation that depends both on $\mathbf{P}$ and on $\sigma_{j}$. The sequence of related ground eigenvectors, $\left\{\phi_{\mathbf{P}}^{\sigma_{j}}\right\}$, that we construct by iteration, turns out to be useful to characterize the original sequence, $\left\{\psi_{\mathbf{P}}^{\sigma_{j}}\right\}$, in two respects:

- the strong convergence of $\left\{\phi_{\mathbf{P}}^{\sigma_{j}}\right\}$ in $F^{b}$ and the fact that the $\sigma_{j}$-dependent coherent transformation is not unitary in the limit $j \rightarrow \infty$ easily imply the weak limit to zero, in $F^{b}$, for the sequence $\left\{\psi_{\mathbf{P}}^{\sigma_{j}}\right\}$;

- by the regularity property in $\mathbf{P}$ of the vectors $\phi_{\mathbf{P}}^{\sigma_{j}}$, uniformly in $j$, and the explicit knowledge of the coherent transformation, we have a better control on the $\mathbf{P}$-dependence of the vectors $\psi_{\mathbf{P}}^{\sigma_{j}}$ and of related quantities. 
Derivation of the coherent factor

Let us assume that $\psi_{\mathbf{P}}$ is an eigenvector of $H_{\mathbf{P}}$ and that it is "coherent in the infrared region", namely

$$
b(\mathbf{k}) \psi_{\mathbf{P}} \approx f_{\mathbf{P}}(\mathbf{k}) \psi_{\mathbf{P}} \quad \text { for } \mathbf{k} \rightarrow 0
$$

where the meaning of the limit is given only "a posteriori". Then the coherent function $f_{\mathbf{P}}(\mathbf{k})$ has to fulfill the following relation:

$$
\left(\psi_{\mathbf{P}},\left[H_{\mathbf{P}}, b(\mathbf{k})\right] \psi_{\mathbf{P}}\right)=0 \quad \text { for } \mathbf{k} \rightarrow 0
$$

The expected behavior for $f_{\mathbf{P}}(\mathbf{k})$ turns out to be

$$
f_{\mathbf{P}}(\mathbf{k}) \approx_{\mathbf{k} \rightarrow 0}-\frac{g}{\sqrt{2|\mathbf{k}|}} \cdot \frac{1}{\left(|\mathbf{k}|+\frac{|\mathbf{k}|^{2}}{2 m}-\frac{\mathbf{P} \cdot \mathbf{k}}{m}+\frac{\mathbf{k} \cdot\left(\psi_{\mathbf{P}}, \mathbf{P}^{p h} \psi_{\mathbf{P}}\right)}{m \cdot\left\|\psi_{\mathbf{P}}\right\|^{2}}\right)},
$$

the conjectured coherent factor is therefore labelled by

$$
\mathbf{P}-\left\langle\mathbf{P}^{p h}\right\rangle_{\psi_{\mathbf{P}}}, \quad\left\langle\mathbf{P}^{p h}\right\rangle_{\psi_{\mathbf{P}}}:=\frac{\left(\psi_{\mathbf{P}}, \mathbf{P}^{p h} \psi_{\mathbf{P}}\right)}{\left\|\psi_{\mathbf{P}}\right\|^{2}}
$$

The heuristic argument indicates that, if the ground state is "coherent in the infrared region", it does not belong to the Fock space. Starting from this result, we essentially perform the proper coherent transformation on the variables $\left\{b(\mathbf{k}), b^{\dagger}(\mathbf{k})\right\}$ of the Hamiltonian $H_{\mathbf{P}}$, and we search for a ground state of the so transformed Hamiltonian in $F^{b}$. Actually, we accomplish this reducing the infrared cutoff step by step.

\subsection{Transformed Hamiltonians}

Let us consider the coherent transformation

$$
b(\mathbf{k}) \longrightarrow b(\mathbf{k})-\frac{g}{\sqrt{2}|\mathbf{k}|^{\frac{3}{2}}\left(1-\widehat{\mathbf{k}} \cdot \frac{\mathbf{P}-\left\langle\mathbf{P}^{p h}\right\rangle_{\psi_{\mathbf{P}}}^{\sigma_{j}}}{m}\right)} \quad \text { for } \mathbf{k}: \quad \sigma_{j} \leq|\mathbf{k}| \leq \kappa
$$

obtained by the unitary operator

$$
W_{\sigma_{j}}\left(\frac{\mathbf{P}-\left\langle\mathbf{P}^{p h}\right\rangle_{\psi_{\mathbf{P}}^{\sigma_{j}}}}{m}\right)=\exp \left[-g \int_{\sigma_{j}}^{\kappa} \frac{b(\mathbf{k})-b^{\dagger}(\mathbf{k})}{|\mathbf{k}|\left(1-\widehat{\mathbf{k}} \cdot \frac{\mathbf{P}-\left\langle\mathbf{P}^{p h}\right\rangle_{\psi_{\mathbf{P}}}^{\sigma_{j}}}{m}\right)} \frac{d^{3} k}{\sqrt{2|\mathbf{k}|}}\right]
$$

which becomes an intertwiner between inequivalent representations (of $\left\{b(\mathbf{k}), b^{\dagger}(\mathbf{k})\right\}$ ) in the limit $j \rightarrow \infty$. 
From the perturbation of the isolated eigenvalue $E_{\mathbf{P}}^{\sigma_{j}}$ of $\left.H_{\mathbf{P}, \sigma_{j}}\right|_{F_{\sigma_{j}}^{+}}$(see [Fr.1]), one can easily check that $\mathbf{P}-\left\langle\mathbf{P}^{p h}\right\rangle_{\psi_{\mathbf{P}}^{\sigma_{j}}}$ corresponds to $m \nabla E^{\sigma_{j}}(\mathbf{P})$, where $\left|\nabla E^{\sigma_{j}}(\mathbf{P})\right|<1$ for $\mathbf{P} \in \Sigma$ as proved in Lemma A2 in Appendix.

The gradient $\nabla E^{\sigma_{j}}(\mathbf{P})$ fulfills the equation:

$$
\begin{aligned}
\left\langle\mathbf{P}^{p h}\right\rangle_{\psi_{\mathbf{P}}} \sigma_{j} & =\mathbf{P}-m \nabla E^{\sigma_{j}}(\mathbf{P}) \\
& =\frac{1}{\left\|\phi_{\mathbf{P}}^{\sigma_{j}}\right\|^{2}}\left(\phi_{\mathbf{P}}^{\sigma_{j}}, \Pi_{\mathbf{P}, \sigma_{j}} \phi_{\mathbf{P}}^{\sigma_{j}}\right)+g^{2} \int_{\sigma_{j}}^{\kappa} \frac{\mathbf{k}}{2|\mathbf{k}|^{3}\left(\alpha_{\mathbf{P}}^{\sigma_{j}}(\widehat{\mathbf{k}})\right)^{2}} d^{3} k
\end{aligned}
$$

where $\alpha_{\mathbf{P}}^{\sigma_{j}}(\widehat{\mathbf{k}}):=1-\widehat{\mathbf{k}} \cdot \nabla E^{\sigma_{j}}(\mathbf{P}), \phi_{\mathbf{P}}^{\sigma_{j}}$ is a ground eigenvector, not specified yet, of the transformed Hamiltonian

$$
W_{\sigma_{j}}\left(\nabla E^{\sigma_{j}}(\mathbf{P})\right) H_{\mathbf{P}, \sigma_{j}} W_{\sigma_{j}}^{\dagger}\left(\nabla E^{\sigma_{j}}(\mathbf{P})\right)
$$

and

$$
\Pi_{\mathbf{P}, \sigma_{j}}:=\mathbf{P}^{p h}-g \int_{\sigma_{j}}^{\kappa} \frac{\mathbf{k}\left(b(\mathbf{k})+b^{\dagger}(\mathbf{k})\right)}{\sqrt{2}|\mathbf{k}|^{\frac{3}{2}} \alpha_{\mathbf{P}}^{\sigma_{j}}(\widehat{\mathbf{k}})} d^{3} k .
$$

We want now to provide a more explicit expression for the transformed Hamiltonian

$$
H_{\mathbf{P}, \sigma_{j}}^{w}:=W_{\sigma_{j}}\left(\nabla E^{\sigma_{j}}(\mathbf{P})\right) H_{\mathbf{P}, \sigma_{j}} W_{\sigma_{j}}^{\dagger}\left(\nabla E^{\sigma_{j}}(\mathbf{P})\right)
$$

which takes into account the relation (6).

Let us rewrite $H_{\mathbf{P}, \sigma_{j}}, \mathbf{P}=m \nabla E^{\sigma_{j}}(\mathbf{P})+\left\langle\mathbf{P}^{p h}\right\rangle_{\psi_{\mathbf{P}}^{\sigma_{j}}}$, as

$$
\begin{aligned}
H_{\mathbf{P}, \sigma_{j}} & =\frac{\mathbf{P}^{2}}{2 m}-\frac{\left(m \nabla E^{\sigma_{j}}(\mathbf{P})+\left\langle\mathbf{P}^{p h}\right\rangle_{\psi_{\mathbf{P}}^{\sigma_{j}}}\right) \cdot \mathbf{P}^{p h}}{m}+\frac{\mathbf{P}^{p h^{2}}}{2 m}+g \int_{\sigma_{j}}^{\kappa}\left(b(\mathbf{k})+b^{\dagger}(\mathbf{k})\right) \frac{d^{3} \mathbf{k}}{\sqrt{2|\mathbf{k}|}}+H^{p h} \\
= & \frac{\mathbf{P}^{2}}{2 m}+\frac{\mathbf{P}^{p h^{2}}}{2 m}-\frac{\left\langle\mathbf{P}^{p h}\right\rangle_{\psi_{\mathbf{P}}^{\sigma_{j}}} \cdot \mathbf{P}^{p h}}{m}+\int_{\kappa}^{\infty}\left(|\mathbf{k}|-\mathbf{k} \cdot \nabla E^{\sigma_{j}}(\mathbf{P})\right) b^{\dagger}(\mathbf{k}) b(\mathbf{k}) d^{3} k \\
& +\int_{\sigma_{j}}^{\kappa}\left(|\mathbf{k}|-\mathbf{k} \cdot \nabla E^{\sigma_{j}}(\mathbf{P})\right)\left(b^{\dagger}(\mathbf{k})+\frac{g}{\sqrt{2}|\mathbf{k}|^{\frac{3}{2}} \alpha_{\mathbf{P}}^{\sigma_{j}}(\widehat{\mathbf{k}})}\right)\left(b(\mathbf{k})+\frac{g}{\sqrt{2}|\mathbf{k}|^{\frac{3}{2}} \alpha_{\mathbf{P}}^{\sigma_{j}}(\widehat{\mathbf{k}})}\right) d^{3} k \\
& +\int_{0}^{\sigma_{j}}\left(|\mathbf{k}|-\mathbf{k} \cdot \nabla E^{\sigma_{j}}(\mathbf{P})\right) b^{\dagger}(\mathbf{k}) b(\mathbf{k}) d^{3} k-g^{2} \int_{\sigma_{j}}^{\kappa} \frac{1}{2|\mathbf{k}|^{2} \alpha_{\mathbf{P}}^{\sigma_{j}}(\widehat{\mathbf{k}})} d^{3} k .
\end{aligned}
$$

The arrangement above aims to show the origin of some terms in the transformed Hamiltonian; in particular, for our purposes, it is important to isolate

$$
W_{\sigma_{j}}\left(\nabla E^{\sigma_{j}}(\mathbf{P})\right) \frac{\left(\mathbf{P}^{p h}-\left\langle\mathbf{P}^{p h}\right\rangle_{\psi_{\mathbf{P}}^{\sigma_{j}}}\right)^{2}}{2 m} W_{\sigma_{j}}^{\dagger}\left(\nabla E^{\sigma_{j}}(\mathbf{P})\right)
$$

and to exploit a related structural property later. 
By performing the coherent transformation (5) on $H_{\mathbf{P}, \sigma_{j}}$, we formally obtain:

$$
\begin{aligned}
& \frac{1}{2 m}\left(\mathbf{P}^{p h}-g \int_{\sigma_{j}}^{\kappa} \frac{\mathbf{k}\left(b(\mathbf{k})+b^{\dagger}(\mathbf{k})\right)}{\sqrt{2}|\mathbf{k}|^{\frac{3}{2}} \alpha_{\mathbf{P}}^{\sigma_{j}}(\widehat{\mathbf{k}})} d^{3} k+g^{2} \int_{\sigma_{j}}^{\kappa} \frac{\mathbf{k}}{2|\mathbf{k}|^{3}\left(\alpha_{\mathbf{P}}^{\sigma_{j}}(\widehat{\mathbf{k}})\right)^{2}} d^{3} k\right)^{2} \\
& -\frac{\left\langle\mathbf{P}^{p h}\right\rangle_{\psi_{\mathbf{P}, \sigma_{j}}}}{m} \cdot\left(\mathbf{P}^{p h}-g \int_{\sigma_{j}}^{\kappa} \frac{\mathbf{k}\left(b(\mathbf{k})+b^{\dagger}(\mathbf{k})\right)}{\sqrt{2}|\mathbf{k}|^{\frac{3}{2}} \alpha_{\mathbf{P}}^{\sigma_{j}}(\widehat{\mathbf{k}})} d^{3} k+g^{2} \int_{\sigma_{j}}^{\kappa} \frac{\mathbf{k}}{2|\mathbf{k}|^{3}\left(\alpha_{\mathbf{P}}^{\sigma_{j}}(\widehat{\mathbf{k}})\right)^{2}} d^{3} k\right) \\
& +\int_{0}^{\infty}\left(|\mathbf{k}|-\mathbf{k} \cdot \nabla E^{\sigma_{j}}(\mathbf{P})\right) b^{\dagger}(\mathbf{k}) b(\mathbf{k}) d^{3} k+\frac{\mathbf{P}^{2}}{2 m}-g^{2} \int_{\sigma_{j}}^{\kappa} \frac{1}{2|\mathbf{k}|^{2} \alpha_{\mathbf{P}}^{\sigma_{j}}(\widehat{\mathbf{k}})} d^{3} k .
\end{aligned}
$$

By substitution it corresponds to

$$
\frac{1}{2 m}\left(\Pi_{\mathbf{P}, \sigma_{j}}-\frac{\left(\phi_{\mathbf{P}}^{\sigma_{j}}, \Pi_{\mathbf{P}, \sigma_{j}} \phi_{\mathbf{P}}^{\sigma_{j}}\right)}{\left\|\phi_{\mathbf{P}}^{\sigma_{j}}\right\|^{2}}\right)^{2}+\int_{0}^{\infty}\left(|\mathbf{k}|-\mathbf{k} \cdot \nabla E^{\sigma_{j}}(\mathbf{P})\right) b^{\dagger}(\mathbf{k}) b(\mathbf{k}) d^{3} k+c_{\mathbf{P}}^{\sigma_{j}}
$$

where $c_{\mathbf{P}}^{\sigma_{j}}=\frac{\mathbf{P}^{2}}{2 m}-\frac{1}{2 m}\left[\mathbf{P}-m \nabla E^{\sigma_{j}}(\mathbf{P})\right]^{2}-g^{2} \int_{\sigma_{j}}^{\kappa} \frac{1}{2|\mathbf{k}|^{2} \alpha_{\mathbf{P}}^{\sigma_{j}}(\widehat{\mathbf{k}})} d^{3} k$.

The expression above, not only formally but also from an operatorial point of view, corresponds to $H_{\mathbf{P}, \sigma_{j}}^{w}$ and its selfadjointness domain (s.a.d.) coincides with $D^{b}\left(H_{\mathbf{P}, \sigma_{j}}\right)$, s.a.d. of $H_{\mathbf{P}, \sigma_{j}}$ (see an analogous proof in [Ne]).

\section{Definitions}

i) To streamline formulas, in next steps we will use the definition

$$
\Gamma_{\mathbf{P}, \sigma_{j}}^{i}:=\Pi_{\mathbf{P}, \sigma_{j}}^{i}-\frac{\left(\phi_{\mathbf{P}}^{\sigma_{j}}, \Pi_{\mathbf{P}, \sigma_{j}}^{i} \phi_{\mathbf{P}}^{\sigma_{j}}\right)}{\left\|\phi_{\mathbf{P}}^{\sigma_{j}}\right\|^{2}} \quad(i=1,2,3 \quad, \quad j \in N) .
$$

ii) In proving the convergence of the sequence $\left\{\phi_{\mathbf{P}}^{\sigma_{j}}\right\}$ (to be constructed) we take advantage of intermediate Hamiltonians, $\widehat{H}_{\mathbf{P}, \sigma_{j}}^{w}$, which are introduced because of the fact that, at fixed $\mathbf{P}(\mathbf{P} \neq 0)$, the $\mathbf{P}$-function in the coherent factor changes step by step:

$$
\begin{aligned}
& \widehat{H}_{\mathbf{P}, \sigma_{j+1}}^{w}:=W_{\sigma_{j+1}}\left(\nabla E^{\sigma_{j}}(\mathbf{P})\right) H_{\mathbf{P}, \sigma_{j+1}} W_{\sigma_{j+1}}^{\dagger}\left(\nabla E^{\sigma_{j}}(\mathbf{P})\right) \\
& =W_{\sigma_{j+1}}\left(\nabla E^{\sigma_{j}}(\mathbf{P})\right) W_{\sigma_{j+1}}^{\dagger}\left(\nabla E^{\sigma_{j+1}}(\mathbf{P})\right) \\
& \quad \times H_{\mathbf{P}, \sigma_{j+1}}^{w} W_{\sigma_{j+1}}\left(\nabla E^{\sigma_{j+1}}(\mathbf{P})\right) W_{\sigma_{j+1}}^{\dagger}\left(\nabla E^{\sigma_{j}}(\mathbf{P})\right) \\
& =\frac{1}{2 m}\left(\Gamma_{\mathbf{P}, \sigma_{j}}-g \int_{\sigma_{j+1}}^{\sigma_{j}} \frac{\mathbf{k}\left(b(\mathbf{k})+b^{\dagger}(\mathbf{k})\right)}{\sqrt{2}|\mathbf{k}|^{\frac{3}{2}} \alpha_{\mathbf{P}}^{\sigma_{j}}(\widehat{\mathbf{k}})} d^{3} k+g^{2} \int_{\sigma_{j+1}}^{\sigma_{j}} \frac{\mathbf{k}}{2|\mathbf{k}|^{3}\left(\alpha_{\mathbf{P}}^{\sigma_{j}}(\widehat{\mathbf{k}})\right)^{2}} d^{3} k\right)^{2} \\
& \quad+\int_{0}^{\infty}\left(|\mathbf{k}|-\mathbf{k} \cdot \nabla E^{\sigma_{j}}(\mathbf{P})\right) b^{\dagger}(\mathbf{k}) b(\mathbf{k}) d^{3} k+\widehat{c}_{\mathbf{P}}^{\sigma_{j+1}} .
\end{aligned}
$$


Here

$$
\widehat{c}_{\mathbf{P}}^{\sigma_{j+1}}=\frac{\mathbf{P}^{2}}{2 m}-\frac{1}{2 m}\left[\mathbf{P}-m \nabla E^{\sigma_{j}}(\mathbf{P})\right]^{2}-g^{2} \int_{\sigma_{j+1}}^{\kappa} \frac{1}{2|\mathbf{k}|^{2} \alpha_{\mathbf{P}}^{\sigma_{j}}(\widehat{\mathbf{k}})} d^{3} k .
$$

They are essentially derived by the same steps used for (7).

iii) Analogously we define:

$$
\begin{aligned}
& \widehat{\Pi}_{\mathbf{P}, \sigma_{j+1}}^{i}:= \\
& =W_{\sigma_{j+1}}\left(\nabla E^{\sigma_{j}}(\mathbf{P})\right) W_{\sigma_{j+1}}^{\dagger}\left(\nabla E^{\sigma_{j+1}}(\mathbf{P})\right) \\
& \times \Pi_{\mathbf{P}, \sigma_{j+1}}^{i} W_{\sigma_{j+1}}\left(\nabla E^{\sigma_{j+1}}(\mathbf{P})\right) W_{\sigma_{j+1}}^{\dagger}\left(\nabla E^{\sigma_{j}}(\mathbf{P})\right) \\
& =P^{p h^{i}}-g \int_{\sigma_{j+1}}^{\kappa} \frac{k^{i}\left(b(\mathbf{k})+b^{\dagger}(\mathbf{k})\right)}{\sqrt{2}|\mathbf{k}|^{\frac{3}{2}} \alpha_{\mathbf{P}}^{\sigma_{j}}(\widehat{\mathbf{k}})} d^{3} k \\
& +\frac{g^{2}}{2} \int_{\sigma_{j+1}}^{\kappa} k^{i}\left[\frac{\left(\alpha_{\mathbf{P}}^{\sigma_{j+1}}(\widehat{\mathbf{k}})\right)^{2}-\left(\alpha_{\mathbf{P}}^{\sigma_{j}}(\widehat{\mathbf{k}})\right)^{2}}{|\mathbf{k}|^{3}\left(\alpha_{\mathbf{P}}^{\sigma_{j}}(\widehat{\mathbf{k}})\right)^{2}\left(\alpha_{\mathbf{P}}^{\sigma_{j+1}}(\widehat{\mathbf{k}})\right)^{2}}\right] d^{3} k \\
& \widehat{\Gamma}_{\mathbf{P}, \sigma_{j+1}}^{i}:=\widehat{\Pi}_{\mathbf{P}, \sigma_{j+1}}^{i}-\frac{\left(\widehat{\phi}_{\mathbf{P}}^{\sigma_{j+1}}, \widehat{\Pi}_{\mathbf{P}, \sigma_{j+1}}^{i} \widehat{\phi}_{\mathbf{P}}^{\sigma_{j+1}}\right)}{\left\|\widehat{\phi}_{\mathbf{P}}^{\sigma_{j+1}}\right\|^{2}},
\end{aligned}
$$

where $\widehat{\phi}_{\mathbf{P}}^{\sigma_{j+1}}$ is ground eigenvector of $\widehat{H}_{\mathbf{P}, \sigma_{j+1}}^{w}$ and is properly defined by the iterative procedure explained in the next paragraph. Note however that, as for $H_{\mathbf{P}, \sigma_{j+1}}^{w}$, the expression is completely defined in terms of the ray of $W_{\sigma_{j+1}}\left(\nabla E^{\sigma_{j}}(\mathbf{P})\right) \psi_{\mathbf{P}}^{\sigma_{j+1}}$.

\section{Remarks}

1) Note that the two transformations

$$
\begin{gathered}
H_{\mathbf{P}, \sigma_{j}} \rightarrow H_{\mathbf{P}, \sigma_{j}}^{w}=W_{\sigma_{j}}\left(\nabla E^{\sigma_{j}}(\mathbf{P})\right) H_{\mathbf{P}, \sigma_{j}} W_{\sigma_{j}}^{\dagger}\left(\nabla E^{\sigma_{j}}(\mathbf{P})\right) \\
H_{\mathbf{P}, \sigma_{j+1}} \rightarrow \widehat{H}_{\mathbf{P}, \sigma_{j+1}}^{w}=W_{\sigma_{j+1}}\left(\nabla E^{\sigma_{j}}(\mathbf{P})\right) H_{\mathbf{P}, \sigma_{j+1}} W_{\sigma_{j+1}}^{\dagger}\left(\nabla E^{\sigma_{j}}(\mathbf{P})\right)
\end{gathered}
$$

are different in the infrared cutoff but not in the $\mathbf{P}$-function inside the coherent factor.

2) The Hamiltonians $H_{\mathbf{P}, \sigma_{j}}, H_{\mathbf{P}, \sigma_{j}}^{w}$ and $\widehat{H}_{\mathbf{P}, \sigma_{j}}^{w}$ are s.a. on the same domain and the formal derivations provided so far are well defined from an operatorial point of view.

\subsection{Convergent sequence}

In order to arrive at a strongly convergent sequence, $\left\{\phi_{\mathbf{P}}^{\sigma_{j}}\right\}$, of ground eigenvectors of $H_{\mathbf{P}, \sigma_{j}}^{w}$, we start from the vector $\phi_{\mathbf{P}}^{\sigma_{0}} \equiv \psi_{0}, \psi_{0}$ vacuum state in $F^{b}$. From the 
results of the previous chapter and by unitarity, the following properties hold for each $j \in N$ (these properties are exploited in Lemma A1, Appendix):

i) $\left.H_{\mathbf{P}, \sigma_{j}}^{w}\right|_{F_{\sigma_{j}}^{+}}$has ground eigenvalue $E_{\mathbf{P}}^{\sigma_{j}}$ with the corresponding gap larger than $\frac{\sigma_{j}}{2}$

ii) $\left.{ }_{\mathbf{P}, \sigma_{j}}^{w}\right|_{F_{\sigma_{j+1}}^{+}}$has ground eigenvalue $E_{\mathbf{P}}^{\sigma_{j}}$ with the corresponding gap larger than $\frac{3}{5} \sigma_{j+1}$.

Comparing the resolvents of the Hamiltonians $H_{\mathbf{P}, \sigma_{j}}^{w}$ and $\widehat{H}_{\mathbf{P}, \sigma_{j+1}}^{w}$, we can construct $\widehat{\phi}_{\mathbf{P}}^{\sigma_{j+1}}$ in terms of a given $\phi_{\mathbf{P}}^{\sigma_{j}}$ by projection, thanks to the estimates contained in Lemma A1, in Appendix, which is the analogue of Lemma 1.3 for the Hamiltonians $H_{\mathbf{P}, \sigma_{j}}$. Therefore we obtain

$$
\widehat{\phi}_{\mathbf{P}}^{\sigma_{j+1}}:=\phi_{\mathbf{P}}^{\sigma_{j}}-\frac{1}{2 \pi i} \oint \sum_{n=1}^{\infty} \frac{1}{H_{\mathbf{P}, \sigma_{j}}^{w}-E_{j+1}}\left(-\left(\Delta H_{\mathbf{P}}^{w}\right)_{\sigma_{j+1}}^{\sigma_{j}} \frac{1}{H_{\mathbf{P}, \sigma_{j}}^{w}-E_{j+1}}\right)^{n} \phi_{\mathbf{P}}^{\sigma_{j}} d E_{j+1}
$$

where

$$
\left(\Delta H_{\mathbf{P}}^{w}\right)_{\sigma_{j+1}}^{\sigma_{j}}:=\widehat{H}_{\mathbf{P}, \sigma_{j+1}}^{w}-{\widehat{c_{\mathbf{P}}}}^{\sigma_{j+1}}+c_{\mathbf{P}}^{\sigma_{j}}-H_{\mathbf{P}, \sigma_{j}}^{w} .
$$

Then we define

$$
\phi_{\mathbf{P}}^{\sigma_{j+1}}:=W_{\sigma_{j+1}}\left(\nabla E^{\sigma_{j+1}}(\mathbf{P})\right) W_{\sigma_{j+1}}^{\dagger}\left(\nabla E^{\sigma_{j}}(\mathbf{P})\right) \widehat{\phi}_{\mathbf{P}}^{\sigma_{j+1}} .
$$

According to this recipe, the construction of $\left\{\phi_{\mathbf{P}}^{\sigma_{j}}\right\}$ is carried out starting from $\phi_{\mathbf{P}}^{\sigma_{0}} \equiv \psi_{0}$ (the corresponding sequence of rays is already known from $\left\{\psi_{\mathbf{P}}^{\sigma_{j}}\right\}$ and the coherent transformation).

Outline of the proof of the convergence. In studying the (strong) convergence of the vectors $\phi_{\mathbf{P}}^{\sigma_{j}}$ for $j \rightarrow \infty$, we have to compare the following vectors one after the other:

$$
\phi_{\mathbf{P}}^{\sigma_{j}} \rightarrow \widehat{\phi}_{\mathbf{P}}^{\sigma_{j+1}} \rightarrow \phi_{\mathbf{P}}^{\sigma_{j+1}} \rightarrow \widehat{\phi}_{\mathbf{P}}^{\sigma_{j+2}} \rightarrow \phi_{\mathbf{P}}^{\sigma_{j+2}}
$$

(in the special case $\mathbf{P}=0$, there is a simplification because $\phi_{\mathbf{P}}^{\sigma_{j}} \equiv \widehat{\phi}_{\mathbf{P}}^{\sigma_{j}}$, being $\nabla E^{\sigma}(0)=0$ by symmetry). First note that one needs a more refined estimate of the difference between the generic vectors $\phi_{\mathbf{P}}^{\sigma_{j}}$ and $\widehat{\phi}_{\mathbf{P}}^{\sigma_{j+1}}$, being the estimates provided in Lemma A1 only sufficient to construct the sequence. At this point a crucial difference emerges with respect the previous sequence, $\left\{\psi_{\mathbf{P}}^{\sigma_{j}}\right\}$. The result of Lemma A1 can be improved, differently from the analogous one (Lemma 1.3) for the sequence $\left\{\psi_{\mathbf{P}}^{\sigma_{j}}\right\}$.

To this purpose break the interaction

$$
\left(\Delta H_{\mathbf{P}}^{w}\right)_{\sigma_{j+1}}^{\sigma_{j}}:=\widehat{H}_{\mathbf{P}, \sigma_{j+1}}^{w}-{\widehat{c_{\mathbf{P}}}}^{\sigma_{j+1}}+c_{\mathbf{P}}^{\sigma_{j}}-H_{\mathbf{P}, \sigma_{j}}^{w}
$$

in

$$
\left[\left(\Delta H_{\mathbf{P}}^{w}\right)_{\sigma_{j+1}}^{\sigma_{j}}\right]^{m i x}+\left[\left(\Delta H_{\mathbf{P}}^{w}\right)_{\sigma_{j+1}}^{\sigma_{j}}\right]^{\text {quad }}
$$


where

$$
\begin{aligned}
& {\left[\left(\Delta H_{\mathbf{P}}^{w}\right)_{\sigma_{j+1}}^{\sigma_{j}}\right]^{m i x}:=} \\
& =\Gamma_{\mathbf{P}, \sigma_{j}} \cdot\left(-\frac{g}{2 m} \int_{\sigma_{j+1}}^{\sigma_{j}} \frac{\mathbf{k}\left(b(\mathbf{k})+b^{\dagger}(\mathbf{k})\right)}{\sqrt{2}|\mathbf{k}|^{\frac{3}{2}} \alpha_{\mathbf{P}}^{\sigma_{j}}(\widehat{\mathbf{k}})} d^{3} k+\frac{g^{2}}{2 m} \int_{\sigma_{j+1}}^{\sigma_{j}} \frac{\mathbf{k}}{2|\mathbf{k}|^{3}\left(\alpha_{\mathbf{P}}^{\sigma_{j}}(\widehat{\mathbf{k}})\right)^{2}} d^{3} k\right)+\text { h.c. }
\end{aligned}
$$

(h.c. means hermitian conjugate) and consider again the expression (8).

Because of the mixed terms, the estimate provided in Lemma A1 for

$$
\left\|\left(\frac{1}{H_{\mathbf{P}, \sigma_{j}}^{w}-E_{j+1}}\right)^{\frac{1}{2}}\left(-\left(\Delta H_{\mathbf{P}}^{w}\right)_{\sigma_{j+1}}^{\sigma_{j}}\right)\left(\frac{1}{H_{\mathbf{P}, \sigma_{j}}^{w}-E_{j+1}}\right)^{\frac{1}{2}}\right\|_{F_{\sigma_{j+1}^{+}}^{+}}
$$

does not imply that the norm $\left\|\widehat{\phi}_{\mathbf{P}}^{\sigma_{j+1}}-\phi_{\mathbf{P}}^{\sigma_{j}}\right\|$ is infinitesimal for $j \rightarrow \infty$. However we are able to give a more refined estimate of the norm $\left\|\hat{\phi}_{\mathbf{P}}^{\sigma_{j+1}}-\phi_{\mathbf{P}}^{\sigma_{j}}\right\|$ by a careful analysis of the first factor in each term of the sum in (8), precisely

$$
\left(\frac{1}{H_{\mathbf{P}, \sigma_{j}}^{w}-E_{j+1}}\right)^{\frac{1}{2}}\left(-\left(\Delta H_{\mathbf{P}}^{w}\right)_{\sigma_{j+1}}^{\sigma_{j}}\right)\left(\frac{1}{H_{\mathbf{P}, \sigma_{j}}^{w}-E_{j+1}}\right) \phi_{\mathbf{P}}^{\sigma_{j}}
$$

Note, indeed, that if an inequality like the following were true

$$
\left\|\left(\frac{1}{H_{\mathbf{P}, \sigma_{j}}^{w}-E_{j+1}}\right)^{\frac{1}{2}}\left[\left(\Delta H_{\mathbf{P}}^{w}\right)_{\sigma_{j+1}}^{\sigma_{j}}\right]^{m i x}\left(\frac{1}{H_{\mathbf{P}, \sigma_{j}}^{w}-E_{j+1}}\right)^{\frac{1}{2}} \phi_{\mathbf{P}}^{\sigma_{j}}\right\| \leq \frac{\epsilon^{\frac{j+1}{8}}}{4}
$$

we could estimate $\left\|\hat{\phi}_{\mathbf{P}}^{\sigma_{j+1}}-\phi_{\mathbf{P}}^{\sigma_{j}}\right\|$ less or equal to $\epsilon^{\frac{j+1}{8}}$. It is sufficient because:

- from Lemma A1, the norm of the contribution due to the quadratic terms can be bounded by $\frac{\epsilon^{\frac{j+1}{8}}}{40}$ (for a proper $g$ )

$$
\left\|\left(\frac{1}{H_{\mathbf{P}, \sigma_{j}}^{w}-E_{j+1}}\right)^{\frac{1}{2}}\left[\left(\Delta H_{\mathbf{P}}^{w}\right)_{\sigma_{j+1}}^{\sigma_{j}}\right]^{q u a d}\left(\frac{1}{H_{\mathbf{P}, \sigma_{j}}^{w}-E_{j+1}}\right)^{\frac{1}{2}} \phi_{\mathbf{P}}^{\sigma_{j}}\right\| \leq \frac{\epsilon^{\frac{j+1}{8}}}{40}
$$

- for each term of the sum, the norms of the other factors of the product are smaller than 1, in particular less than $\frac{1}{12}$ according to Lemma A1. 
Therefore from (9) we would have

$$
\begin{aligned}
& \left\|\sum_{n=1}^{\infty} \frac{1}{H_{\mathbf{P}, \sigma_{j}}^{w}-E_{j+1}}\left(-\left(\Delta H_{\mathbf{P}}^{w}\right)_{\sigma_{j+1}}^{\sigma_{j}} \frac{1}{H_{\mathbf{P}, \sigma_{j}}^{w}-E_{j+1}}\right)^{n} \phi_{\mathbf{P}}^{\sigma_{j}}\right\| \\
& \leq\left|\left(\frac{1}{E_{\mathbf{P}}^{\sigma_{j}}-E_{j+1}}\right)\right|^{\frac{1}{2}} \cdot\left\|\left(\frac{1}{H_{\mathbf{P}, \sigma_{j}}^{w}-E_{j+1}}\right)^{\frac{j}{4}}\right\|_{F_{\sigma_{j+1}}^{+}} \cdot \epsilon^{\frac{j+1}{8}} \cdot\left(\frac{11}{40}\right) \cdot \sum_{n=1}^{\infty}\left(\frac{1}{12}\right)^{n-1} \\
& \leq\left|\left(\frac{1}{E_{\mathbf{P}}^{\sigma_{j}}-E_{j+1}}\right)\right| \cdot \epsilon^{\frac{j+1}{8}} .
\end{aligned}
$$

Finally, as it is shown in Corollary 2.4, an estimate like $\left\|\widehat{\phi}_{\mathbf{P}}^{\sigma_{j+1}}-\phi_{\mathbf{P}}^{\sigma_{j}}\right\| \leq \epsilon^{\frac{j+1}{8}}$ implies the convergence of the sequence $\left\{\phi_{\mathbf{P}}^{\sigma_{j}}\right\}$.

The conclusion of the previous reasoning is that, turning to a strong estimate for the first factor in all the terms of the series expansion in (8), we are able to prove the convergence of the sequence if the inequality (9) holds.

Taking care of the expression of $\left[\left(\Delta H_{\mathbf{P}}^{w}\right)_{\sigma_{j+1}}^{\sigma_{j}}\right]^{m i x}$, after few steps and for $g$ sufficiently small, one can check that the inequality (9) is implied by the following

$$
g^{2}\left|\left(\Gamma_{\mathbf{P}, \sigma_{j}}^{i} \phi_{\mathbf{P}}^{\sigma_{j}},\left(\frac{1}{H_{\mathbf{P}, \sigma_{j}}^{w}-E_{j+1}}\right) \Gamma_{\mathbf{P}, \sigma_{j}}^{i} \phi_{\mathbf{P}}^{\sigma_{j}}\right)\right|<\frac{M}{\epsilon^{\frac{j}{4}}} \quad(i=1,2,3)
$$

$\left(E_{j+1}\right.$ is s.t. $\left.\left|E_{j+1}-E_{\mathbf{P}}^{\sigma_{j}}\right|=\frac{11}{20} \sigma_{j+1}\right)$ where $M$ is a sufficiently small constant, that is uniform in $j$.

We arrive at this conclusion through two technical lemmas: Lemma 2.1 and Lemma 2.2 .

In Lemma 2.1, starting from the spectral representation of the operator $H_{\mathbf{P}, \sigma_{j}}^{w}$, we provide a form bound of the type

$$
\left(\varphi,\left|\frac{1}{H_{\mathbf{P}, \sigma_{j}}^{w}-E_{j+1}}\right| \varphi\right) \leq a \cdot\left|\left(\varphi, \frac{1}{H_{\mathbf{P}, \sigma_{j}}^{w}-E_{j+1}} \varphi\right)\right|
$$

where $a>0$ is uniform in $j$, for $\varphi$ belonging to the subspace of $F_{\sigma_{j}}^{+}$orthogonal to the ground eigenvector, $\phi_{\mathbf{P}}^{\sigma_{j}}$, of $H_{\mathbf{P}, \sigma_{j}}^{w}$. This argument can be applied to the vectors $\Gamma_{\mathbf{P}, \sigma_{j}}^{i} \phi_{\mathbf{P}}^{\sigma_{j}} \quad(i=1,2,3)$ because the property

$$
\Gamma_{\mathbf{P}, \sigma_{j}}^{i} \phi_{\mathbf{P}}^{\sigma_{j}}=\left\{\Pi_{\mathbf{P}, \sigma_{j}}^{i} \phi_{\mathbf{P}}^{\sigma_{j}}-\frac{1}{\left\|\phi_{\mathbf{P}}^{\sigma_{j}}\right\|^{2}} \cdot\left(\phi_{\mathbf{P}}^{\sigma_{j}}, \Pi_{\mathbf{P}, \sigma_{j}}^{i} \phi_{\mathbf{P}}^{\sigma_{j}}\right) \phi_{\mathbf{P}}^{\sigma_{j}}\right\} \perp \phi_{\mathbf{P}}^{\sigma_{j}}
$$

holds by definition. 
In Lemma 2.2 we deal with the relevant term in (9); by calling a term "relevant" we mean that the other ones have a better infrared behavior or can be reduced to the relevant term plus smaller terms. The relevant term, for $j \geq 1$, turns out to be

$$
\left\|\left(\frac{1}{H_{\mathbf{P}, \sigma_{j}}^{w}-E_{j+1}}\right)^{\frac{1}{2}} \frac{g}{m} \cdot \int_{\sigma_{j+1}}^{\sigma_{j}} \frac{k^{i} b^{\dagger}(\mathbf{k})}{\sqrt{2}|\mathbf{k}|^{\frac{3}{2}} \alpha_{\mathbf{P}}^{\sigma_{j}}(\widehat{\mathbf{k}})} d^{3} k \Gamma_{\mathbf{P}, \sigma_{j}}^{i}\left(\frac{1}{H_{\mathbf{P}, \sigma_{j}}^{w}-E_{j+1}}\right)^{\frac{1}{2}} \phi_{\mathbf{P}}^{\sigma_{j}}\right\| .
$$

In the estimate of the relevant term, we essentially exploit the pull-through formula (see [B.F.S]) combined with the result of Lemma 2.1, in order to pull the operator

$$
\int_{\sigma_{j+1}}^{\sigma_{j}} \frac{k^{i} b^{\dagger}(\mathbf{k})}{\sqrt{2}|\mathbf{k}|^{\frac{3}{2}} \alpha_{\mathbf{P}}^{\sigma_{j}}(\widehat{\mathbf{k}})} d^{3} k
$$

through the resolvent from the right side. In Lemma 2.2 we assume the ratio $\frac{\kappa}{m}$ sufficiently small. Being standard computations, the lemma is proved in Appendix (Lemma A3).

The final step is Theorem 2.3, in which, by induction, we provide the estimate (10) and then the inequality (9).

Lemma 2.1 The following inequalities hold:

I) $\left(\int_{\sigma_{j+1}}^{\sigma_{j}} \frac{k^{i} b^{\dagger}(\mathbf{k})}{\sqrt{2}|\mathbf{k}|^{\frac{3}{2}} \alpha_{\mathbf{P}}^{\sigma_{j}}(\widehat{\mathbf{k}})} d^{3} k \Gamma_{\mathbf{P}, \sigma_{j}}^{i} \phi_{\mathbf{P}}^{\sigma_{j}},\left|\frac{1}{H_{\mathbf{P}, \sigma_{j}}^{w}-E_{j+1}}\right| \int_{\sigma_{j+1}}^{\sigma_{j}} \frac{k^{i} b^{\dagger}(\mathbf{k})}{\sqrt{2}|\mathbf{k}|^{\frac{3}{2}} \alpha_{\mathbf{P}}^{\sigma_{j}}(\widehat{\mathbf{k}})} d^{3} k \Gamma_{\mathbf{P}, \sigma_{j}}^{i} \phi_{\mathbf{P}}^{\sigma_{j}}\right)$ $\leq \sqrt{122}\left|\left(\int_{\sigma_{j+1}}^{\sigma_{j}} \frac{k^{i} b^{\dagger}(\mathbf{k})}{\sqrt{2}|\mathbf{k}|^{\frac{3}{2}} \alpha_{\mathbf{P}}^{\sigma_{j}}(\widehat{\mathbf{k}})} d^{3} k \Gamma_{\mathbf{P}, \sigma_{j}}^{i} \phi_{\mathbf{P}}^{\sigma_{j}}, \frac{1}{H_{\mathbf{P}, \sigma_{j}}^{w}-E_{j+1}} \int_{\sigma_{j+1}}^{\sigma_{j}} \frac{k^{i} b^{\dagger}(\mathbf{k})}{\sqrt{2}|\mathbf{k}|^{\frac{3}{2}} \alpha_{\mathbf{P}}^{\sigma_{j}}(\widehat{\mathbf{k}})} d^{3} k \Gamma_{\mathbf{P}, \sigma_{j}}^{i} \phi_{\mathbf{P}}^{\sigma_{j}}\right)\right|$ II) $\left(\Gamma_{\mathbf{P}, \sigma_{j}}^{i} \phi_{\mathbf{P}}^{\sigma_{j}},\left|\frac{1}{H_{\mathbf{P}, \sigma_{j}}^{w}-E_{j+1}}\right| \Gamma_{\mathbf{P}, \sigma_{j}}^{i} \phi_{\mathbf{P}}^{\sigma_{j}}\right) \leq Q(\epsilon) \cdot\left|\left(\Gamma_{\mathbf{P}, \sigma_{j}}^{i} \phi_{\mathbf{P}}^{\sigma_{j}}, \frac{1}{H_{\mathbf{P}, \sigma_{j}}^{w}-E_{j+1}} \Gamma_{\mathbf{P}, \sigma_{j}}^{i} \phi_{\mathbf{P}}^{\sigma_{j}}\right)\right|$ where $Q(\epsilon) \equiv \sqrt{1+\left(\frac{11 \sqrt{\epsilon}}{10-11 \sqrt{\epsilon}}\right)^{2}}$.

Proof. Let us define the wave functions, $\zeta_{I}(z), \zeta_{I I}(z)$, of

$$
\int_{\sigma_{j+1}}^{\sigma_{j}} \frac{k^{i} b^{\dagger}(\mathbf{k})}{\sqrt{2}|\mathbf{k}|^{\frac{3}{2}} \alpha_{\mathbf{P}}^{\sigma_{j}}(\widehat{\mathbf{k}})} d^{3} k \Gamma_{\mathbf{P}, \sigma_{j}}^{i} \phi_{\mathbf{P}}^{\sigma_{j}} \quad \text { and } \quad \Gamma_{\mathbf{P}, \sigma_{j}}^{i} \phi_{\mathbf{P}}^{\sigma_{j}}
$$

respectively, in the spectral variable, $z$, of the operator $H_{\mathbf{P}, \sigma_{j}}^{w}-R e E_{j+1}$ (we do not explicit the other degrees of freedom).

Note that:

- the operator $H_{\mathbf{P}, \sigma_{j}}^{w}-R e E_{j+1}$, applied to the vector

$$
\int_{\sigma_{j+1}}^{\sigma_{j}} \frac{k^{i} b^{\dagger}(\mathbf{k})}{\sqrt{2}|\mathbf{k}|^{\frac{3}{2}} \alpha_{\mathbf{P}}^{\sigma_{j}}(\widehat{\mathbf{k}})} d^{3} k \Gamma_{\mathbf{P}, \sigma_{j}}^{i} \phi_{\mathbf{P}}^{\sigma_{j}}
$$


takes spectral values larger or equal to $\frac{1}{20} \sigma_{j+1}\left(=\frac{3}{5} \sigma_{j+1}-\frac{11}{20} \sigma_{j+1}\right)$ because of Lemma 1.1 and the property

$$
\int_{\sigma_{j+1}}^{\sigma_{j}} \frac{k^{i} b^{\dagger}(\mathbf{k})}{\sqrt{2}|\mathbf{k}|^{\frac{3}{2}} \alpha_{\mathbf{P}}^{\sigma_{j}}(\widehat{\mathbf{k}})} d^{3} k \Gamma_{\mathbf{P}, \sigma_{j}}^{i} \phi_{\mathbf{P}}^{\sigma_{j}} \perp \phi_{\mathbf{P}}^{\sigma_{j}}
$$

- the operator $H_{\mathbf{P}, \sigma_{j}}^{w}-R e E_{j+1}$ takes spectral values larger or equal to $\frac{10-11 \sqrt{\epsilon}}{20} \sigma_{j}\left(=\frac{1}{2} \sigma_{j}-\frac{11}{20} \sigma_{j+1}\right)$ when it is applied to the vector $\Gamma_{\mathbf{P}, \sigma_{j}}^{i} \phi_{\mathbf{P}}^{\sigma_{j}}$; it follows from the gap estimate (concerning $\left.H_{\mathbf{P}, \sigma_{j}}^{w}\right|_{F_{\sigma_{j}}^{+}}$) contained in Theorem 1.4 and the orthogonality property.

Let us write the scalar products I) and II), in the statement of the lemma, by using the spectral representation of the operator $H_{\mathbf{P}, \sigma_{j}}^{w}-R e E_{j+1}$ with the given spectral measure $d \mu(z)$. We do not make the integration over the remaining degrees of freedom explicit. In the chosen spectral representation and because of the range of the variable $z$, the following inequalities are clear:

$$
\begin{aligned}
& \left|\int \frac{\left|\zeta_{I, I I}(z)\right|^{2}}{z-i \operatorname{Im}\left(E_{j+1}\right)} d \mu(z)\right| \\
& \geq\left|\int \frac{\left|\zeta_{I, I I}(z)\right|^{2}}{\sqrt{z^{2}+\left[\operatorname{Im}\left(E_{j+1}\right)\right]^{2}}} \cdot \frac{z}{\sqrt{z^{2}+\left[\operatorname{Im}\left(E_{j+1}\right)\right]^{2}}} d \mu(z)\right| \\
& \geq \frac{z_{\min }}{\sqrt{z_{\text {min }}^{2}+\left|E_{j+1}-E_{\mathbf{P}}^{\sigma_{j}}\right|^{2}}} \cdot \int \frac{\left|\zeta_{I, I I}(z)\right|^{2}}{\sqrt{z^{2}+\left[\operatorname{Im}\left(E_{j+1}\right)\right]^{2}}} d \mu(z)
\end{aligned}
$$

It follows that:

- in the case I), being $z_{\min } \geq \frac{1}{20} \sigma_{j+1}$ and then

$$
\frac{z_{\min }}{\sqrt{z_{\min }^{2}+\left|E_{j+1}-E_{\mathbf{P}}^{\sigma_{j}}\right|^{2}}} \geq \frac{1}{\sqrt{122}}
$$

we have

$$
\int \frac{\left|\zeta_{I}(z)\right|^{2}}{\sqrt{z^{2}+\left[\operatorname{Im}\left(E_{j+1}\right)\right]^{2}}} d \mu(z) \leq \sqrt{122} \cdot\left|\int \frac{\left|\zeta_{I}(z)\right|^{2}}{z-i \operatorname{Im}\left(E_{j+1}\right)} d \mu(z)\right|
$$

- in the case II), being $z_{m i n} \geq \frac{10-11 \sqrt{\epsilon}}{20} \cdot \sigma_{j}$ and then

$$
\frac{z_{\min }}{\sqrt{z_{\min }^{2}+\left|E_{j+1}-E_{\mathbf{P}}^{\sigma_{j}}\right|^{2}}} \geq \frac{1}{\sqrt{1+\left(\frac{11 \sqrt{\epsilon}}{10-11 \sqrt{\epsilon}}\right)^{2}}}
$$


we have

$$
\int \frac{\left|\zeta_{I I}(z)\right|^{2}}{\sqrt{z^{2}+\left[\operatorname{Im}\left(E_{j+1}\right)\right]^{2}}} d \mu(z) \leq Q(\epsilon) \cdot\left|\int \frac{\left|\zeta_{I I}(z)\right|^{2}}{z-i \operatorname{Im}\left(E_{j+1}\right)} d \mu(z)\right|
$$

The two inequalities correspond to the ones in the statement of the lemma.

Lemma 2.2 For a sufficiently small ratio $\frac{\kappa}{m}$, in addition to the constructive hypotheses, the following inequality holds:

$$
\begin{aligned}
& \left\|\left(\frac{1}{H_{\mathbf{P}, \sigma_{j}}^{w}-E_{j+1}}\right)^{\frac{1}{2}} \int_{\sigma_{j+1}}^{\sigma_{j}} \frac{k^{i} b^{\dagger}(\mathbf{k})}{\sqrt{2}|\mathbf{k}|^{\frac{3}{2}} \alpha_{\mathbf{P}}^{\sigma_{j}}(\widehat{\mathbf{k}})} d^{3} k \Gamma_{\mathbf{P}, \sigma_{j}}^{i}\left(\frac{1}{H_{\mathbf{P}, \sigma_{j}}^{w}-E_{j+1}}\right)^{\frac{1}{2}} \phi_{\mathbf{P}}^{\sigma_{j}}\right\|^{2} \\
& \leq 2 Q(\epsilon) \cdot \sqrt{122} \cdot Z_{\sigma_{j+1}}^{\sigma_{j}} \cdot\left|\frac{1}{E_{\mathbf{P}}^{\sigma_{j}}-E_{j+1}}\right| \cdot\left|\left(\Gamma_{\mathbf{P}, \sigma_{j}}^{i} \phi_{\mathbf{P}}^{\sigma_{j}},\left(\frac{1}{H_{\mathbf{P}, \sigma_{j}}^{w}-E_{j+1}}\right) \Gamma_{\mathbf{P}, \sigma_{j}}^{i} \phi_{\mathbf{P}}^{\sigma_{j}}\right)\right|
\end{aligned}
$$

being

$$
Z_{\sigma_{j+1}}^{\sigma_{j}}=\left\{\sum_{i} \int_{\sigma_{j+1}}^{\sigma_{j}} \frac{k^{i^{2}}}{2|\mathbf{k}|^{3} \alpha_{\mathbf{P}}^{\sigma_{j}}(\widehat{\mathbf{k}})^{2}} d^{3} k\right\} .
$$

Proof. See Lemma A3 in Appendix.

Theorem 2.3 For $g$ and $\frac{\kappa}{m}$ sufficiently small, the inequality (10)

$$
g^{2} \cdot\left|\left(\Gamma_{\mathbf{P}, \sigma_{j}}^{i} \phi_{\mathbf{P}}^{\sigma_{j}},\left(\frac{1}{H_{\mathbf{P}, \sigma_{j}}^{w}-E_{j+1}}\right) \Gamma_{\mathbf{P}, \sigma_{j}}^{i} \phi_{\mathbf{P}}^{\sigma_{j}}\right)\right|<\frac{M}{\epsilon^{\frac{j}{4}}} \quad(i=1,2,3)
$$

holds uniformly in $j$ and in $\mathbf{P} \in \Sigma$, being $M$ a sufficiently small constant.

Proof. We recall that, due to the result of Lemma 2.2 and the preliminary discussion about the convergence, the inequality above, for $M$ (and $g$ ) sufficiently small, implies the estimate (9) in Outline of the proof of the convergence, namely:

$$
\left\|\left(\frac{1}{H_{\mathbf{P}, \sigma_{j}}^{w}-E_{j+1}}\right)^{\frac{1}{2}}\left[\left(\Delta H_{\mathbf{P}}^{w}\right)_{\sigma_{j+1}}^{\sigma_{j}}\right]^{m i x}\left(\frac{1}{E_{\mathbf{P}}^{\sigma_{j}}-E_{j+1}}\right)^{\frac{1}{2}} \phi_{\mathbf{P}}^{\sigma_{j}}\right\| \leq \frac{\epsilon^{\frac{j+1}{8}}}{4}
$$

and then the bound $\left\|\hat{\phi}_{\mathbf{P}}^{\sigma_{j+1}}-\phi_{\mathbf{P}}^{\sigma_{j}}\right\| \leq \epsilon^{\frac{j+1}{8}}$ as previously discussed.

In order to prove the inequality (10) in the statement of the present theorem, first we relate the expression corresponding to the level $j$ to the one corresponding to $j-1$. 
1) We start applying the unitary operator

$$
W_{\sigma_{j}}\left(\nabla E^{\sigma_{j-1}}(\mathbf{P})\right) W_{\sigma_{j}}^{\dagger}\left(\nabla E^{\sigma_{j}}(\mathbf{P})\right)
$$

to both the factors of the scalar product to obtain the analogous quantity with the corresponding "^- operators":

$$
\left(\widehat{\Gamma}_{\mathbf{P}, \sigma_{j}}^{i} \widehat{\phi}_{\mathbf{P}}^{\sigma_{j}},\left(\frac{1}{\widehat{H}_{\mathbf{P}, \sigma_{j}}^{w}-E_{j+1}}\right) \widehat{\Gamma}_{\mathbf{P}, \sigma_{j}}^{i} \widehat{\phi}_{\mathbf{P}}^{\sigma_{j}}\right) .
$$

Note now that the circle of integration $C_{j+1}$, related to the integration variable $E_{j+1}$, has a radius, $r=\left|E_{j+1}-E_{\mathbf{P}}^{\sigma_{j}}\right|=\frac{11}{20} \kappa \epsilon^{\frac{j+1}{2}}$, where $\epsilon$ is a fixed parameter in the construction. Therefore, thanks to the result of Lemma $1.2, E_{\mathbf{P}}^{\sigma_{j-1}}$ is inside $C_{j+1}$ for $g$ sufficiently small (in particular of order $\epsilon$ ). In this respect see the figure below.

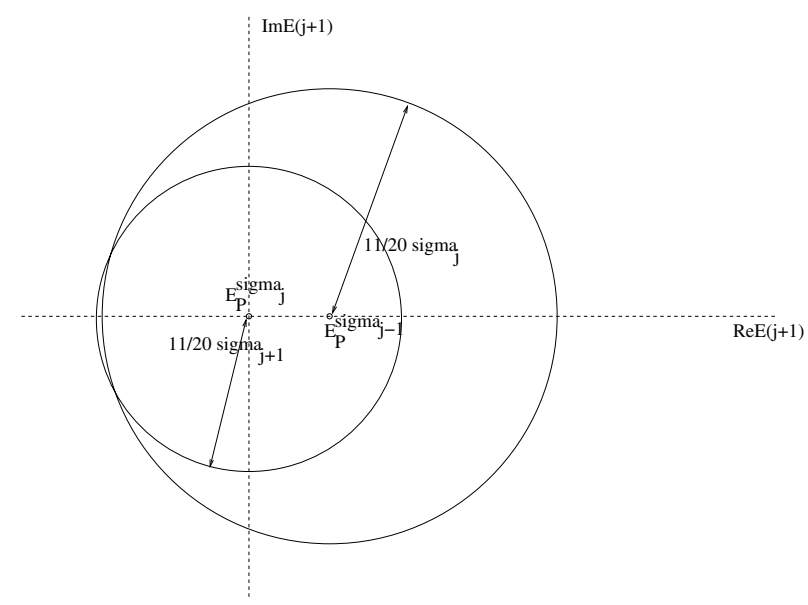

By the same procedure used in Lemma A1 (in Appendix), taking into account Lemma 1.2 and for $g$ sufficiently small, it is possible to perform a series expansion to eventually arrive at the following bound for the absolute value of the expression (11):

$$
2 \cdot\left(\widehat{\Gamma}_{\mathbf{P}, \sigma_{j}}^{i} \widehat{\phi}_{\mathbf{P}}^{\sigma_{j}},\left|\frac{1}{H_{\mathbf{P}, \sigma_{j-1}}^{w}-E_{j+1}}\right| \widehat{\Gamma}_{\mathbf{P}, \sigma_{j}}^{i} \widehat{\phi}_{\mathbf{P}}^{\sigma_{j}}\right) .
$$

2) Adding and subtracting $\Gamma_{\mathbf{P}, \sigma_{j-1}}^{i} \phi_{\mathbf{P}}^{\sigma_{j-1}}$ on the left and on the right of the scalar product above, we bound the new terms that we get, using elementary properties 
of the scalar product:

$$
\begin{aligned}
& 2 g^{2} \cdot\left(\widehat{\Gamma}_{\mathbf{P}, \sigma_{j}}^{i} \widehat{\phi}_{\mathbf{P}}^{\sigma_{j}},\left|\frac{1}{H_{\mathbf{P}, \sigma_{j-1}}^{w}-E_{j+1}}\right| \widehat{\Gamma}_{\mathbf{P}, \sigma_{j}}^{i} \widehat{\phi}_{\mathbf{P}}^{\sigma_{j}}\right) \\
& \leq 4 g^{2} \cdot\left(\widehat{\Gamma}_{\mathbf{P}, \sigma_{j}}^{i} \widehat{\phi}_{\mathbf{P}}^{\sigma_{j}}-\Gamma_{\mathbf{P}, \sigma_{j-1}}^{i} \phi_{\mathbf{P}}^{\sigma_{j-1}},\left|\frac{1}{H_{\mathbf{P}, \sigma_{j-1}}^{w}-E_{j+1}}\right|\left(\widehat{\Gamma}_{\mathbf{P}, \sigma_{j}}^{i} \widehat{\phi}_{\mathbf{P}}^{\sigma_{j}}-\Gamma_{\mathbf{P}, \sigma_{j-1}}^{i} \phi_{\mathbf{P}}^{\sigma_{j-1}}\right)\right) \\
& \quad+4 g^{2} \cdot\left(\Gamma_{\mathbf{P}, \sigma_{j-1}}^{i} \phi_{\mathbf{P}}^{\sigma_{j-1}},\left|\frac{1}{H_{\mathbf{P}, \sigma_{j-1}}^{w}-E_{j+1}}\right| \Gamma_{\mathbf{P}, \sigma_{j-1}}^{i} \phi_{\mathbf{P}}^{\sigma_{j-1}}\right) .
\end{aligned}
$$

At this point, we want to reduce the quantity (13) to the expression (10) at the level $j-1$, times a constant less than $\epsilon^{-\frac{1}{4}}$, and to estimate the remainder (12) by a quantity of order $\epsilon^{-\frac{j}{4}}$. It requires some technical manipulation and an inductive argument that eventually leads to the thesis.

The proof consists in some preliminary results before the induction, and it is so organized:

i) Treatment of the remainder (12);

ii) Treatment of the expression (13);

iii) Estimate of $\left\|\phi_{\mathbf{P}}^{\sigma_{j}}-\widehat{\phi}_{\mathbf{P}}^{\sigma_{j}}\right\|$;

iv) Inductive proof.

Treatment of the remainder (12)

The following inequality holds

$$
\begin{aligned}
& 4 g^{2} \cdot\left(\widehat{\Gamma}_{\mathbf{P}, \sigma_{j}}^{i} \widehat{\phi}_{\mathbf{P}}^{\sigma_{j}}-\Gamma_{\mathbf{P}, \sigma_{j-1}}^{i} \phi_{\mathbf{P}}^{\sigma_{j-1}},\left|\frac{1}{H_{\mathbf{P}, \sigma_{j-1}}^{w}-E_{j+1}}\right|\left(\widehat{\Gamma}_{\mathbf{P}, \sigma_{j}}^{i} \widehat{\phi}_{\mathbf{P}}^{\sigma_{j}}-\Gamma_{\mathbf{P}, \sigma_{j-1}}^{i} \phi_{\mathbf{P}}^{\sigma_{j-1}}\right)\right) \\
& \leq 8 g^{2} \cdot\left\|\left(\frac{1}{H_{\mathbf{P}, \sigma_{j-1}}^{w}-E_{j+1}}\right)^{\frac{1}{2}}\left(\widehat{\Gamma}_{\mathbf{P}, \sigma_{j}}^{i} \widehat{\phi}_{\mathbf{P}}^{\sigma_{j}}-\Gamma_{\mathbf{P}, \sigma_{j-1}}^{i} \widehat{\phi}_{\mathbf{P}}^{\sigma_{j}}\right)\right\|^{2} \\
& \quad+8 g^{2} \cdot\left\|\left(\frac{1}{H_{\mathbf{P}, \sigma_{j-1}}^{w}-E_{j+1}}\right)^{\frac{1}{2}} \Gamma_{\mathbf{P}, \sigma_{j-1}}^{i}\left(\widehat{\phi}_{\mathbf{P}}^{\sigma_{j}}-\phi_{\mathbf{P}}^{\sigma_{j-1}}\right)\right\|^{2} \\
& \leq \frac{R_{1}(g)}{\epsilon^{\frac{j}{4}}} \cdot\left(\frac{\left\|\widehat{\phi}_{\mathbf{P}}^{\sigma_{j}}-\phi_{\mathbf{P}}^{\sigma_{j-1}}\right\|+\epsilon^{\frac{j}{8}}}{2 \epsilon^{\frac{j}{8}}}\right)^{2}+\frac{R_{2}(g)}{\epsilon^{\frac{j}{4}}} \cdot\left(\frac{\left\|\frac{\widehat{\phi}_{\mathbf{P}}^{\sigma_{j}}}{\| \hat{\phi}_{\mathbf{P}}^{\sigma_{j}}}\right\|}{\frac{\phi_{\mathbf{P}}^{\sigma_{j-1}}}{\left\|\phi_{\mathbf{P}}^{\sigma_{j-1}}\right\|} \|+\epsilon^{\frac{j}{8}}}\right)^{2}
\end{aligned}
$$

where $R_{1}(g)$ and $R_{2}(g)$ are independent of $j$ and vanish for $g \rightarrow 0 . R_{1}(g)$ and $R_{2}(g)$ are obtained considering the following facts: 
i) from the relation (6) and the definition of $\widehat{\Pi}_{\mathbf{P}, \sigma_{j+1}}^{i}$ in paragraph 2.1:

$$
\begin{aligned}
\widehat{\Gamma}_{\mathbf{P}, \sigma_{j}}^{i}= & \Gamma_{\mathbf{P}, \sigma_{j-1}}^{i}-g \int_{\sigma_{j}}^{\sigma_{j-1}} \frac{k^{i}\left(b(\mathbf{k})+b^{\dagger}(\mathbf{k})\right)}{\sqrt{2}|\mathbf{k}|^{\frac{3}{2}} \alpha_{\mathbf{P}}^{\sigma_{j-1}}(\widehat{\mathbf{k}})} d^{3} k \\
& +m\left(\nabla^{i} E^{\sigma_{j}}(\mathbf{P})-\nabla^{i} E^{\sigma_{j-1}}(\mathbf{P})\right)+g^{2} \int_{\sigma_{j}}^{\sigma_{j-1}} \frac{k^{i}}{2|\mathbf{k}|^{3}\left(\alpha_{\mathbf{P}}^{\sigma_{j-1}}(\widehat{\mathbf{k}})\right)^{2}} d^{3} k
\end{aligned}
$$

ii) $\left\|\left(\frac{1}{H_{\mathbf{P}, \sigma_{j-1}}^{w}-E_{j+1}}\right)^{\frac{1}{2}}\left(\widehat{\Gamma}_{\mathbf{P}, \sigma_{j}}^{i} \widehat{\phi}_{\mathbf{P}}^{\sigma_{j}}-\Gamma_{\mathbf{P}, \sigma_{j-1}}^{i} \widehat{\phi}_{\mathbf{P}}^{\sigma_{j}}\right)\right\|$

$$
\begin{gathered}
\leq\left\|\left(\frac{1}{H_{\mathbf{P}, \sigma_{j-1}}^{w}-E_{j+1}}\right)^{\frac{1}{2}}\left(g \int_{\sigma_{j}}^{\sigma_{j-1}} \frac{k^{i}\left(b(\mathbf{k})+b^{\dagger}(\mathbf{k})\right)}{\sqrt{2}|\mathbf{k}|^{\frac{3}{2}} \alpha_{\mathbf{P}}^{\sigma_{j-1}}(\widehat{\mathbf{k}})} d^{3} k \widehat{\phi}_{\mathbf{P}}^{\sigma_{j}}\right)\right\| \\
+\left|m \nabla E^{\sigma_{j}}(\mathbf{P})-m \nabla E^{\sigma_{j-1}}(\mathbf{P})\right| \cdot\left\|\left(\frac{1}{H_{\mathbf{P}, \sigma_{j-1}}^{w}-E_{j+1}}\right)^{\frac{1}{2}} \widehat{\phi}_{\mathbf{P}}^{\sigma_{j}}\right\| \\
+\left|g^{2} \int_{\sigma_{j}}^{\sigma_{j-1}} \frac{k^{i}}{2|\mathbf{k}|^{3} \alpha_{\mathbf{P}}^{\sigma_{j-1}}(\widehat{\mathbf{k}})} d^{3} k\right| \cdot\left\|\left(\frac{1}{H_{\mathbf{P}, \sigma_{j-1}}^{w}-E_{j+1}}\right)^{\frac{1}{2}} \widehat{\phi}_{\mathbf{P}}^{\sigma_{j}}\right\|
\end{gathered}
$$

iii) the following estimates hold with constants $C^{\nabla E}, C^{\prime}, C^{\prime \prime}$, uniform in $j$, for $g$ sufficiently small

- $\left|\nabla E^{\sigma_{j}}(\mathbf{P})-\nabla E^{\sigma_{j-1}}(\mathbf{P})\right| \leq C^{\nabla E} \cdot\left(\left\|\frac{\hat{\phi}_{\mathrm{P}}^{\sigma_{j}}}{\left\|\hat{\phi}_{\mathbf{P}}^{\sigma_{j}}\right\|}-\frac{\phi_{\mathrm{P}}^{\sigma_{j-1}}}{\left\|\phi_{\mathbf{P}}^{\sigma_{j-1}}\right\|}\right\|+\epsilon^{\frac{j}{8}}\right)$

(see Lemma A2 )

- $\left\|\int_{\sigma_{j}}^{\sigma_{j-1}} \frac{k^{i}\left(b(\mathbf{k})+b^{\dagger}(\mathbf{k})\right)}{\sqrt{2}|\mathbf{k}|^{\frac{3}{2}} \alpha_{\mathbf{P}}^{\sigma_{j}-1}(\widehat{\mathbf{k}})} d^{3} k\left(\frac{1}{H_{\mathrm{P}, \sigma_{j-1}}^{w}-E_{j+1}}\right)^{\frac{1}{2}}\right\|_{F_{\sigma_{j}}^{+}} \leq C^{\prime} \cdot \epsilon^{\frac{j}{4}}$

$\left\|\left(\frac{1}{H_{\mathbf{P}, \sigma_{j-1}}^{w}-E_{j+1}}\right)^{\frac{1}{2}} \Gamma_{\mathbf{P}, \sigma_{j-1}}^{i}\right\|_{F_{\sigma_{j}}^{+}} \leq \frac{C^{\prime \prime}}{\epsilon^{\frac{3}{4}}}$

by steps as in Lemma A1 and for the result in Lemma 1.2.

Treatment of the expression (13)

In the next estimate, once we are given a sufficiently small $g$, we proceed as follows.

We exploit the crucial property

$$
\left(\phi_{\mathbf{P}}^{\sigma_{j-1}}, \Gamma_{\mathbf{P}, \sigma_{j-1}}^{i} \phi_{\mathbf{P}}^{\sigma_{j-1}}\right)=0
$$


so that we can apply Lemma 2.1 in a slightly modified version, getting a multiplicative factor $Q(\epsilon)$. Then we expand

$$
\left(\frac{1}{H_{\mathbf{P}, \sigma_{j-1}}^{w}-E_{j+1}}\right)
$$

in terms of $\left(\frac{1}{H_{\mathbf{P}, \sigma_{j-1}}-E_{j}}\right)$ and $E_{j+1}-E_{j}$, thanks to the spectral consequence of the orthogonality property; in this way we get a multiplicative factor $b$ ( $b \preceq 2$ ) uniformly (in $j$ ) bounded from above. A further application of Lemma 2.1 provides another factor $Q(\epsilon)$. Namely:

$$
\begin{aligned}
& 4 g^{2} \cdot\left(\Gamma_{\mathbf{P}, \sigma_{j-1}}^{i} \phi_{\mathbf{P}}^{\sigma_{j-1}},\left|\left(\frac{1}{H_{\mathbf{P}, \sigma_{j-1}}^{w}-E_{j}}\right)\right| \Gamma_{\mathbf{P}, \sigma_{j-1}}^{i} \phi_{\mathbf{P}}^{\sigma_{j-1}}\right) \\
& \leq 4 g^{2} \cdot Q(\epsilon) \cdot\left|\left(\Gamma_{\mathbf{P}, \sigma_{j-1}}^{i} \phi_{\mathbf{P}}^{\sigma_{j-1}},\left(\frac{1}{H_{\mathbf{P}, \sigma_{j-1}}^{w}-E_{j+1}}\right) \Gamma_{\mathbf{P}, \sigma_{j-1}}^{i} \phi_{\mathbf{P}}^{\sigma_{j-1}}\right)\right| \\
& \leq 4 g^{2} \cdot b \cdot Q^{2}(\epsilon) \cdot\left|\left(\Gamma_{\mathbf{P}, \sigma_{j-1}}^{i} \phi_{\mathbf{P}}^{\sigma_{j-1}},\left(\frac{1}{H_{\mathbf{P}, \sigma_{j-1}}^{w}-E_{j}}\right) \Gamma_{\mathbf{P}, \sigma_{j-1}}^{i} \phi_{\mathbf{P}}^{\sigma_{j-1}}\right)\right|
\end{aligned}
$$

Remark. The expression above corresponds, at the level $j-1$, to what we want to estimate (see (10)) times the factor $4 \cdot b \cdot Q^{2}(\epsilon)$. Acting on $g$, at fixed $\epsilon$, we can provide a multiplicative factor, $4 \cdot b \cdot Q^{2}(\epsilon)$, less than $\epsilon^{-\frac{1}{4}}(>1)$. This fact is crucial in the inductive proof. It is enough because we only require a divergent (for $j \rightarrow \infty$ ) bound for the expression (10).

Estimate of $\left\|\phi_{\mathbf{P}}^{\sigma_{j}}-\widehat{\phi}_{\mathbf{P}}^{\sigma_{j}}\right\|$

Before the inductive proof, a further preliminary step is in order, concerning with an upper bound for the norm $\left\|\phi_{\mathbf{P}}^{\sigma_{j}}-\widehat{\phi}_{\mathbf{P}}^{\sigma_{j}}\right\|$. It is the second part of the step

$$
\phi_{\mathbf{P}}^{\sigma_{j-1}} \rightarrow \widehat{\phi}_{\mathbf{P}}^{\sigma_{j}} \rightarrow \phi_{\mathbf{P}}^{\sigma_{j}}
$$

and it can be easily related to the first one $\left(\phi_{\mathbf{P}}^{\sigma_{j-1}} \rightarrow \widehat{\phi}_{\mathbf{P}}^{\sigma_{j}}\right)$ through the variation of the energy gradient as it is explained below.

Note that

$$
\phi_{\mathbf{P}}^{\sigma_{j}} \equiv W_{\sigma_{j}}\left(\nabla E^{\sigma_{j}}(\mathbf{P})\right) W_{\sigma_{j}}^{\dagger}\left(\nabla E^{\sigma_{j-1}}(\mathbf{P})\right) \widehat{\phi}_{\mathbf{P}}^{\sigma_{j}}
$$

by definition, from which

$$
\begin{aligned}
\left\|\phi_{\mathbf{P}}^{\sigma_{j}}-\widehat{\phi}_{\mathbf{P}}^{\sigma_{j}}\right\| & =\left\|W_{\sigma_{j}}\left(\nabla E^{\sigma_{j}}(\mathbf{P})\right) W_{\sigma_{j}}^{\dagger}\left(\nabla E^{\sigma_{j-1}}(\mathbf{P})\right) \widehat{\phi}_{\mathbf{P}}^{\sigma_{j}}-\widehat{\phi}_{\mathbf{P}}^{\sigma_{j}}\right\| \\
& =\left\|W_{\sigma_{j}}^{\dagger}\left(\nabla E^{\sigma_{j-1}}(\mathbf{P})\right) W_{\sigma_{j}}\left(\nabla E^{\sigma_{j}}(\mathbf{P})\right) \psi_{\mathbf{P}}^{\sigma_{j}}-\psi_{\mathbf{P}}^{\sigma_{j}}\right\|
\end{aligned}
$$


An upper estimate of the norm above is therefore given by

$$
g \cdot Z \cdot\left|\nabla E_{\mathbf{P}}^{\sigma_{j}}-\nabla E_{\mathbf{P}}^{\sigma_{j-1}}\right| \cdot\left|\ln \left(\epsilon^{\frac{j}{2}}\right)\right|
$$

where

- $Z$ is a constant dependent on $m, \kappa$ and uniform in $j$;

- the logarithmically divergent (for $j \rightarrow \infty$ ) quantity, $\left|\ln \left(\epsilon^{\frac{j}{2}}\right)\right|$, arises from

$$
\left(\int_{\sigma_{j}}^{\kappa}\left\|b(\mathbf{k}) \psi_{\mathbf{P}, \sigma_{j}}\right\|^{2} d^{3} k\right)^{\frac{1}{2}}
$$

taking into account that

$$
b(\mathbf{k}) \psi_{\mathbf{P}, \sigma_{j}}=\frac{g}{\sqrt{2|\mathbf{k}|}}\left(\frac{1}{E^{\sigma_{j}}(\mathbf{P})-|\mathbf{k}|-H_{\mathbf{P}-\mathbf{k}, \sigma_{j}}}\right) \psi_{\mathbf{P}, \sigma_{j}}
$$

for $\left\{\mathbf{k}: \sigma_{j} \leq|\mathbf{k}| \leq \kappa\right\}$ (proof as in [Fr.1]), $\left\|\psi_{\mathbf{P}, \sigma_{j}}\right\| \leq 1$, and from

$$
\left(\int_{\sigma_{j}}^{\kappa}\left(\frac{1}{\sqrt{2}|\mathbf{k}|^{\frac{3}{2}} \alpha_{\mathbf{P}}^{\sigma_{j-1}}(\widehat{\mathbf{k}}) \cdot \alpha_{\mathbf{P}}^{\sigma_{j}}(\widehat{\mathbf{k}})}\right)^{2} d^{3} k\right)^{\frac{1}{2}}
$$

- the infinitesimal (for $j \rightarrow \infty$ ) quantity

$$
\left|\nabla E_{\mathbf{P}}^{\sigma_{j}}-\nabla E_{\mathbf{P}}^{\sigma_{j-1}}\right|
$$

comes from the difference between the coherent factors in the Weyl operators.

Inductive proof

Now, let $g$ be sufficiently small such that:

- $G_{1}^{\infty}:=\sum_{k=1}^{\infty} g \cdot Z \cdot 4 C^{\nabla E} \cdot \epsilon^{\frac{k}{8}}\left|\ln \left(\epsilon^{\frac{k}{2}}\right)\right| \leq \frac{1}{12}$

- $\left\|\widehat{\phi}_{\mathbf{P}}^{\sigma_{1}}-\phi_{\mathbf{P}}^{\sigma_{0}}\right\| \leq \epsilon^{\frac{1}{8}}$

- the bound (10) is valid for $j=1$, the previously established, uniform in $j$, constraints hold, in particular we require

$$
0<R_{1}(g)+R_{2}(g)=: R(g) \leq\left(1-4 b Q^{2}(\epsilon) \epsilon^{\frac{1}{4}}\right) \cdot M
$$

where $4 b \cdot Q^{2}(\epsilon) \epsilon^{\frac{1}{4}}<1$, being $b \preceq 2$ and $0<\epsilon<\left(\frac{1}{5}\right)^{8}$. 


\section{Inductive hypotheses}

Let us assume that for the chosen value of $g$ the property (10) holds for $j-1(>1)$ and that

$$
\left\|\phi_{\mathbf{P}}^{\sigma_{j-1}}-\phi_{\mathbf{P}}^{\sigma_{0}}\right\| \leq \sum_{k=1}^{j-1} \epsilon^{\frac{k}{8}}+G_{1}^{j-1}
$$

where $\phi_{\mathbf{P}}^{\sigma_{0}} \equiv \psi_{0}\left(\right.$ vacuum state in $\left.F^{b}\right)$ and $G_{1}^{j-1}=g \cdot \sum_{k=1}^{j-1} Z \cdot 4 C^{\nabla E} \cdot \epsilon^{\frac{k}{8}}\left|\ln \left(\epsilon^{\frac{k}{2}}\right)\right|$.

\section{Thesis}

As consequences of the inductive hypotheses:

- $\left\|\widehat{\phi}_{\mathbf{P}}^{\sigma_{j}}-\phi_{\mathbf{P}}^{\sigma_{j-1}}\right\| \leq \epsilon^{\frac{j}{8}}$

- since $\left\|\phi_{\mathbf{P}}^{\sigma_{j-1}}\right\| \geq 1-\left\|\phi_{\mathbf{P}}^{\sigma_{j-1}}-\phi_{\mathbf{P}}^{\sigma_{0}}\right\|>1-\sum_{k=1}^{\infty} \epsilon^{\frac{k}{8}}-G_{1}^{\infty}>\frac{2}{3}$

$$
\left\|\frac{\widehat{\phi}_{\mathbf{P}}^{\sigma_{j}}}{\left\|\widehat{\phi}_{\mathbf{P}}^{\sigma_{j}}\right\|}-\frac{\phi_{\mathbf{P}}^{\sigma_{j-1}}}{\left\|\phi_{\mathbf{P}}^{\sigma_{j-1}}\right\|}\right\| \leq 2\left\|\frac{\widehat{\phi}_{\mathbf{P}}^{\sigma_{j}}-\phi_{\mathbf{P}}^{\sigma_{j-1}}}{\left\|\phi_{\mathbf{P}}^{\sigma_{j-1}}\right\|}\right\| \leq 3 \epsilon^{\frac{j}{8}}
$$

- $\left|\nabla E_{\mathbf{P}}^{\sigma_{j}}-\nabla E_{\mathbf{P}}^{\sigma_{j-1}}\right| \leq 4 C^{\nabla E} \cdot \epsilon^{\frac{j}{8}}$ (see Lemma A2).

Then starting from the equality

$$
\left|\left(\Gamma_{\mathbf{P}, \sigma_{j}}^{i} \phi_{\mathbf{P}}^{\sigma_{j}},\left(\frac{1}{H_{\mathbf{P}, \sigma_{j}}^{w}-E_{j+1}}\right) \Gamma_{\mathbf{P}, \sigma_{j}}^{i} \phi_{\mathbf{P}}^{\sigma_{j}}\right)\right|=\left|\left(\widehat{\Gamma}_{\mathbf{P}, \sigma_{j}}^{i} \widehat{\phi}_{\mathbf{P}}^{\sigma_{j}},\left(\frac{1}{\widehat{H}_{\mathbf{P}, \sigma_{j}}^{w}-E_{j+1}}\right) \widehat{\Gamma}_{\mathbf{P}, \sigma_{j}}^{i} \widehat{\phi}_{\mathbf{P}}^{\sigma_{j}}\right)\right|
$$

and collecting the results obtained so far (for the remainder (12) and the expression (13)), we have

$$
\begin{aligned}
& g^{2}\left|\left(\Gamma_{\mathbf{P}, \sigma_{j}}^{i} \phi_{\mathbf{P}}^{\sigma_{j}},\left(\frac{1}{H_{\mathbf{P}, \sigma_{j}}^{w}-E_{j+1}}\right) \Gamma_{\mathbf{P}, \sigma_{j}}^{i} \phi_{\mathbf{P}}^{\sigma_{j}}\right)\right| \\
& \leq \frac{R(g)}{\epsilon^{\frac{j}{4}}}+4 g^{2} b \cdot Q^{2}(\epsilon) \cdot\left|\left(\Gamma_{\mathbf{P}, \sigma_{j-1}}^{i} \phi_{\mathbf{P}}^{\sigma_{j-1}},\left(\frac{1}{H_{\mathbf{P}, \sigma_{j-1}}^{w}-E_{j}}\right) \Gamma_{\mathbf{P}, \sigma_{j-1}}^{i} \phi_{\mathbf{P}}^{\sigma_{j-1}}\right)\right| \\
& \leq \frac{R(g)}{\epsilon^{\frac{j}{4}}}+4 b \cdot Q^{2}(\epsilon) \cdot \frac{M}{\epsilon^{\frac{j-1}{4}}} \leq \frac{M}{\epsilon^{\frac{j}{4}}} .
\end{aligned}
$$

At the same time:

$$
\begin{aligned}
\left\|\phi_{\mathbf{P}}^{\sigma_{j}}-\phi_{\mathbf{P}}^{\sigma_{0}}\right\| & \leq\left\|\widehat{\phi}_{\mathbf{P}}^{\sigma_{j}}-\phi_{\mathbf{P}}^{\sigma_{j}}\right\|+\left\|\widehat{\phi}_{\mathbf{P}}^{\sigma_{j}}-\phi_{\mathbf{P}}^{\sigma_{j-1}}\right\|+\left\|\phi_{\mathbf{P}}^{\sigma_{j-1}}-\phi_{\mathbf{P}}^{\sigma_{0}}\right\| \\
& \leq g \cdot Z \cdot 4 C^{\nabla E} \cdot \epsilon^{\frac{j}{8}}\left|\ln \left(\epsilon^{\frac{j}{2}}\right)\right|+\epsilon^{\frac{j}{8}}+\sum_{k=1}^{j-1} \epsilon^{\frac{k}{8}}+G_{1}^{j-1}
\end{aligned}
$$

which means

$$
\left\|\phi_{\mathbf{P}}^{\sigma_{j}}-\phi_{\mathbf{P}}^{\sigma_{0}}\right\| \leq \sum_{k=1}^{j} \epsilon^{\frac{k}{8}}+G_{1}^{j}
$$


Corollary 2.4 Given the result of Theorem 2.3, the sequence $\left\{\phi_{\mathbf{P}}^{\sigma_{j}}\right\}\left(\phi_{\mathbf{P}}^{\sigma_{0}} \equiv \psi_{0}, \psi_{0}\right.$ vacuum state in $F^{b}$ ) converges strongly to a non-vanishing vector.

Proof. By the estimates of Theorem 2.3 we easily conclude that $\left\{\phi_{\mathbf{P}}^{\sigma_{j}}\right\}$ is a Cauchy sequence:

$$
\left\|\phi_{\mathbf{P}}^{\sigma_{l}}-\phi_{\mathbf{P}}^{\sigma_{j}}\right\| \leq \sum_{k=j+1}^{l} \epsilon^{\frac{k}{8}}+G_{j+1}^{l} \quad \forall l, j \in N \quad l \geq j+1
$$

The limit does not vanish because

$$
\left\|\phi_{\mathbf{P}}^{\sigma_{j}}\right\| \geq 1-\left(\frac{\epsilon^{\frac{1}{8}}}{1-\epsilon^{\frac{1}{8}}}+G_{1}^{\infty}\right) \geq \frac{2}{3} .
$$

\section{Regularity}

In this section we define a normalized vector $\phi_{\mathbf{P}}^{\sigma}$, that is ground state of $\left.H_{\mathbf{P}, \sigma}^{w}\right|_{F_{\sigma}^{+}}$ $(\sigma \leq \kappa \epsilon)$, for $\sigma$ in the continuum. Starting from infrared sequences of cutoffs which fill the continuum, we construct a ground eigenvector for each $\sigma, \bar{\phi}_{\mathbf{P}}^{\sigma}$, by the projection method performed in the previous section. Having fixed the ground state of $\left.H_{\mathbf{P}, \sigma}^{w}\right|_{F_{\sigma}^{+}}$, we define the vector $\phi_{\mathbf{P}}^{\sigma}$, by applying the one-dimensional projection, corresponding to the ray, on the vacuum state (in $F^{b}$ ) $\psi_{0}$ and normalizing. To do it, in advance we check that $\left(\bar{\phi}_{\mathbf{P}}^{\sigma}, \psi_{0}\right) \neq 0$.

By this procedure we get:

- the strong convergence of the so-defined vector, $\phi_{\mathbf{P}}^{\sigma}$, to a vector $\phi_{\mathbf{P}}$, for $\sigma \rightarrow 0$;

- the Hölder property with respect to $\mathbf{P}$ :

$$
\left\|\phi_{\mathbf{P}+\Delta \mathbf{P}}^{\sigma}-\phi_{\mathbf{P}}^{\sigma}\right\| \leq C_{\delta}|\Delta \mathbf{P}|^{\frac{1}{16}-\delta}
$$

for $\mathbf{P}, \mathbf{P}+\Delta \mathbf{P} \in \Sigma$ and $\Delta \mathbf{P} \in I$, where $I \subset \Sigma$ is a fixed ball around $\Delta \mathbf{P}=0$, for $\delta>0$ and where $C_{\delta}$ is uniform in $\mathbf{P}, \Delta \mathbf{P}$ and $\sigma$.

Preliminary conditions

Let us consider the infrared sequences starting from $\left\{\kappa \epsilon^{\prime}: \kappa \epsilon \geq \kappa \epsilon^{\prime} \geq \kappa \epsilon \sqrt{\epsilon}\right\}$ and sufficiently small values of $g$ and $\frac{\kappa}{m}$ such that it is possible to perform the iterative procedure uniformly in $\epsilon^{\prime}, \epsilon \geq \epsilon^{\prime} \geq \epsilon \sqrt{\epsilon}$, and in $\mathbf{P} \in \Sigma$, with the properties already shown in the case of the factor $\epsilon$. Therefore we can assume the results of Theorem 2.3 and Corollary 2.4 for the factor $\epsilon^{\prime}$. We also require that for the chosen value of the coupling constant $g$ :

$$
\left|\left(\phi_{\mathbf{P}}^{\kappa \epsilon^{\prime}}, \psi_{0}\right)\right|>\frac{1}{3} \quad \forall \epsilon^{\prime}, \epsilon \geq \epsilon^{\prime} \geq \epsilon \sqrt{\epsilon}, \forall \mathbf{P} \in \Sigma .
$$


Definition of $\bar{\phi}_{\mathbf{P}}^{\sigma}$

Given a $\sigma$ ranging between $\sigma_{j}$ and $\sigma_{j+1}, j \geq 2$, we can always write it as $\kappa \epsilon^{\prime}(\sigma)^{\frac{j}{2}}$ where

$$
\epsilon^{\prime}(\sigma):=\left(\frac{\sigma}{\kappa}\right)^{\frac{2}{j}}
$$

By performing the iteration shown in the previous section, we define

$$
\bar{\phi}_{\mathbf{P}}^{\sigma}:=\phi_{\mathbf{P}}^{\kappa \epsilon^{\prime}(\sigma)^{\frac{j}{2}}} .
$$

Lemma 3.1 $\left(\bar{\phi}_{\mathbf{P}}^{\sigma}, \psi_{0}\right) \neq 0 \quad \forall \sigma \leq \kappa \epsilon, \forall \mathbf{P} \in \Sigma$.

Proof. Knowing that $\left\|\phi_{\mathbf{P}}^{\kappa \epsilon^{\prime}}-\phi_{\mathbf{P}}^{\kappa \epsilon^{\prime} \frac{j}{2}}\right\| \leq \frac{1}{3}$ (from Corollary 2.4) and being $\left\|\psi_{0}\right\|=1$, we have:

$$
\left|\left(\bar{\phi}_{\mathbf{P}}^{\sigma}, \psi_{0}\right)\right| \geq||\left(\phi_{\mathbf{P}}^{\kappa \epsilon^{\prime}(\sigma)}, \psi_{0}\right)|-|\left(\bar{\phi}_{\mathbf{P}}^{\sigma}-\phi_{\mathbf{P}}^{\kappa \epsilon^{\prime}(\sigma)}, \psi_{0}\right) \|>0 .
$$

Definition of $\phi_{\mathbf{P}}^{\sigma}$

Since $\bar{\phi}_{\mathbf{P}}^{\sigma}$ is ground eigenvector of $\left.H_{\mathbf{P}, \sigma}^{w}\right|_{F_{\sigma}^{+}}$with a gap larger than $\frac{\sigma}{2}$ by construction, thanks to Lemma 3.1 we can define the normalized vector

$$
\phi_{\mathbf{P}}^{\sigma}:=\frac{-\frac{1}{2 \pi i} \oint \frac{1}{H_{\mathbf{P}, \sigma}^{w}-E} d E \psi_{0}}{\left\|-\frac{1}{2 \pi i} \oint \frac{1}{H_{\mathbf{P}, \sigma}^{w}-E} d E \psi_{0}\right\|}
$$

(where $E \in \mathcal{C}$ and s.t. $\left|E-E_{\mathbf{P}}^{\sigma}\right|=\frac{\sigma}{4}$ ) that is ground state of $\left.H_{\mathbf{P}, \sigma}^{w}\right|_{F_{\sigma}^{+}}$.

Theorem 3.2 For $\mathbf{P} \in \Sigma$, the limit $s-\lim _{\sigma \rightarrow 0} \phi_{\mathbf{P}}^{\sigma}=: \phi_{\mathbf{P}}$ exists.

Proof. Again write $\bar{\phi}_{\mathbf{P}}^{\sigma_{2}}-\bar{\phi}_{\mathbf{P}}^{\sigma_{1}}$ in the following way

$$
\bar{\phi}_{\mathbf{P}}^{\sigma_{2}}-\bar{\phi}_{\mathbf{P}}^{\sigma_{1}}=\bar{\phi}_{\mathbf{P}}^{\sigma_{2}}-\phi_{\mathbf{P}}^{\kappa \epsilon^{\prime}\left(\sigma_{2}\right)^{\frac{l}{2}}}+\phi_{\mathbf{P}}^{\kappa \epsilon^{\prime}\left(\sigma_{2}\right)^{\frac{l}{2}}}-\phi_{\mathbf{P}}^{\kappa \epsilon^{\prime}\left(\sigma_{1}\right)^{\frac{m}{2}}}+\phi_{\mathbf{P}}^{\kappa \epsilon^{\prime}\left(\sigma_{1}\right)^{\frac{m}{2}}}-\bar{\phi}_{\mathbf{P}}^{\sigma_{1}}
$$

where $l, m$ are natural numbers.

Now, given an arbitrarily small $\rho>0$, there exist natural numbers $l, m$ sufficiently large and a phase $e^{i \eta(\rho)}$ for which

$$
\left\|\frac{\phi_{\mathbf{P}}^{\kappa \epsilon^{\prime}\left(\sigma_{1}\right)^{\frac{m}{2}}}}{\left\|\phi_{\mathbf{P}}^{\kappa \epsilon^{\prime}\left(\sigma_{1}\right)^{\frac{m}{2}}}\right\|}-e^{i \eta(\rho)} \frac{\phi_{\mathbf{P}}^{\kappa \epsilon^{\prime}\left(\sigma_{2}\right)^{\frac{l}{2}}}}{\left\|\phi_{\mathbf{P}}^{\kappa \epsilon^{\prime}\left(\sigma_{2}\right)^{\frac{l}{2}}}\right\|}\right\| \leq \rho .
$$

This is essentially due to the convergence established in Corollary 2.4 and because of the fact that the ground state is unique as long as there is a cutoff, by construction. 
Therefore we can estimate

$$
\left\|\frac{\bar{\phi}_{\mathbf{P}}^{\sigma_{2}}}{\left\|\bar{\phi}_{\mathbf{P}}^{\sigma_{2}}\right\|}-e^{-i \eta(\rho)} \frac{\bar{\phi}_{\mathbf{P}}^{\sigma_{1}}}{\left\|\bar{\phi}_{\mathbf{P}}^{\sigma_{1}}\right\|}\right\| \leq c_{\delta}\left(\left(\frac{\sigma_{2}}{\kappa}\right)^{\frac{1}{4}-\delta}+\left(\frac{\sigma_{1}}{\kappa}\right)^{\frac{1}{4}-\delta}+\rho\right)
$$

with $\delta>0$ and arbitrarily small, and $c_{\delta}$ a $\delta$-dependent constant.

Moreover we have that

$$
\begin{aligned}
& \left\|\frac{\bar{\phi}_{\mathbf{P}}^{\sigma_{1}}}{\left\|\bar{\phi}_{\mathbf{P}}^{\sigma_{1}}\right\|} \cdot\left(\frac{\bar{\phi}_{\mathbf{P}}^{\sigma_{1}}}{\left\|\bar{\phi}_{\mathbf{P}}^{\sigma_{1}}\right\|}, \psi_{0}\right)-\frac{\bar{\phi}_{\mathbf{P}}^{\sigma_{2}}}{\left\|\bar{\phi}_{\mathbf{P}}^{\sigma_{2}}\right\|} \cdot\left(\frac{\bar{\phi}_{\mathbf{P}}^{\sigma_{2}}}{\left\|\bar{\phi}_{\mathbf{P}}^{\sigma_{2}}\right\|}, \psi_{0}\right)\right\| \\
& \leq\left\|\frac{\bar{\phi}_{\mathbf{P}}^{\sigma_{2}}}{\left\|\bar{\phi}_{\mathbf{P}}^{\sigma_{2}}\right\|}-e^{-i \eta(\rho)} \frac{\bar{\phi}_{\mathbf{P}}^{\sigma_{1}}}{\left\|\bar{\phi}_{\mathbf{P}}^{\sigma_{1}}\right\|}\right\| \cdot\left(\|\left(\frac{\bar{\phi}_{\mathbf{P}}^{\sigma_{1}}}{\left\|\bar{\phi}_{\mathbf{P}}^{\sigma_{1}}\right\|}, \psi_{0}\right) \mid+1\right)
\end{aligned}
$$

Hence it follows that $\phi_{\mathbf{P}}^{\sigma}\left(\equiv \frac{\bar{\phi}_{\mathbf{P}}^{\sigma}\left(\bar{\phi}_{\mathbf{P}}^{\sigma}, \psi_{0}\right)}{\left\|\bar{\phi}_{\mathbf{P}}^{\sigma}\left(\bar{\phi}_{\mathbf{P}}^{\sigma}, \psi_{0}\right)\right\|}\right)$ converges strongly to a nonzero vector $\phi_{\mathbf{P}}$ in $F^{b}$, with an error of order $\left(\frac{\sigma}{\kappa}\right)^{\frac{1}{4}-\delta}$ at most.

Lemma 3.3 The following Hölder estimate holds:

$$
\left|\nabla E^{\sigma}(\mathbf{P})-\nabla E^{\sigma}(\mathbf{P}+\Delta \mathbf{P})\right| \leq C|\Delta \mathbf{P}|^{\frac{1}{16}}
$$

where the constant $C$ is uniform in $0<\sigma<\kappa \epsilon$, in $\mathbf{P}, \mathbf{P}+\Delta \mathbf{P} \in \Sigma$ and $\Delta \mathbf{P} \in$ $\widehat{I}$, where $\widehat{I}:=\left\{\Delta \mathbf{P}: \frac{|\Delta \mathbf{P}|}{m} \leq\left(\frac{1}{3 C_{\widehat{I}}}\right)^{\frac{8}{3}}, m^{\frac{3}{4}}|\Delta \mathbf{P}|^{\frac{1}{4}} \leq \kappa \epsilon\right\}$ and $C_{\widehat{I}}$ is a constant sufficiently larger than 1.

Proof. The idea is to perturb, in $\mathbf{P}$, the gradient

$$
\nabla E^{|\Delta \mathbf{P}|^{\frac{1}{4}}}(\mathbf{P}) \equiv\left(\psi_{\mathbf{P}}^{|\Delta \mathbf{P}|^{\frac{1}{4}}}, \frac{\mathbf{P}-\mathbf{P}^{p h}}{m} \psi_{\mathbf{P}}^{|\Delta \mathbf{P}|^{\frac{1}{4}}}\right)
$$

where $\psi_{\mathbf{P}}^{|\Delta \mathbf{P}|^{\frac{1}{4}}}$ is the (normalized) ground eigenvector of $H_{\mathbf{P}, m^{\frac{3}{4}}|\Delta \mathbf{P}|^{\frac{1}{4}}}$, which we simply denote as $H_{\mathbf{P},|\Delta \mathbf{P}|^{\frac{1}{4}}}$ (analogous simplified notation for $F_{|\Delta \mathbf{P}|^{\frac{1}{4}}}^{+}$).

For this purpose we use a series expansion of the resolvent

$$
\left.\frac{1}{H_{\mathbf{P}+\Delta \mathbf{P},|\Delta \mathbf{P}|^{\frac{1}{4}}}-E}\right|_{F_{|\Delta \mathbf{P}|^{+}}^{\frac{1}{4}}}
$$

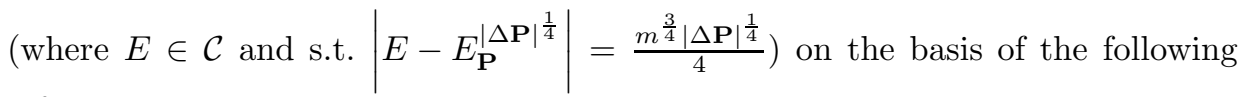
information: 
i) $H_{\mathbf{P}+\Delta \mathbf{P},|\Delta \mathbf{P}|^{\frac{1}{4}}}-H_{\mathbf{P},|\Delta \mathbf{P}|^{\frac{1}{4}}}=-\frac{\Delta \mathbf{P}}{m} \cdot \mathbf{P}^{p h}+\frac{\Delta \mathbf{P}}{m} \cdot \mathbf{P}+\frac{|\Delta \mathbf{P}|^{2}}{2 m}$;

ii) $\left.H_{\mathbf{P},|\Delta \mathbf{P}|^{\frac{1}{4}}}\right|_{F_{|\Delta \mathbf{P}|^{+}}^{+}}$has unique ground state $\psi_{\mathbf{P}}^{|\Delta \mathbf{P}|^{\frac{1}{4}}}$ of energy $E_{\mathbf{P}}^{|\Delta \mathbf{P}|^{\frac{1}{4}}}$ and its gap is bounded from below by $\frac{m^{\frac{3}{4}}|\Delta \mathbf{P}|^{\frac{1}{4}}}{2}$ (Theorem 1.4 in the continuum case);

iii) the quantity

$$
m^{\frac{3}{8}}|\Delta \mathbf{P}|^{\frac{1}{8}} \cdot\left\|\frac{\Delta \mathbf{P} \cdot\left(\mathbf{P}-\mathbf{P}^{p h}\right)}{|\Delta \mathbf{P}| \sqrt{m}}\left(\frac{1}{H_{\mathbf{P},|\Delta \mathbf{P}|^{\frac{1}{4}}}-E}\right)^{\frac{1}{2}}\right\|_{F^{+}}
$$

is uniformly bounded in $\mathbf{P}, \mathbf{P}+\Delta \mathbf{P} \in \Sigma$, therefore we can find a constant $C_{\widehat{I}}$ sufficiently larger than 1 such that

$$
m^{\frac{3}{4}}|\Delta \mathbf{P}|^{\frac{1}{4}} .\left\|\left(\frac{1}{{ }_{\mathbf{P},|\Delta \mathbf{P}|^{\frac{1}{4}}}-E}\right)^{\frac{1}{2}}\left(\frac{\Delta \mathbf{P} \cdot\left(\mathbf{P}-\mathbf{P}^{p h}\right)}{m|\Delta \mathbf{P}|}+\frac{|\Delta \mathbf{P}|}{2 m}\right)\left(\frac{1}{H_{\mathbf{P},|\Delta \mathbf{P}|^{\frac{1}{4}}}-E}\right)^{\frac{1}{2}}\right\|_{F_{|\Delta \mathbf{P}|^{+}}^{\frac{1}{4}}}<C_{\hat{I}} ;
$$

iv) for $\Delta \mathbf{P}$ belonging to $\widehat{I}$ (defined in the statement of the lemma)

$$
\left\|-\frac{1}{2 \pi i} \oint \frac{1}{H_{\mathbf{P}+\Delta \mathbf{P},|\Delta \mathbf{P}|^{\frac{1}{4}}}-E} d E \psi_{\mathbf{P}}^{|\Delta \mathbf{P}|^{\frac{1}{4}}}\right\| \geq 1-\sum_{n=1}^{\infty}\left(\frac{|\Delta \mathbf{P}|^{\frac{3}{4}}}{m^{\frac{3}{4}}} \cdot C_{\widehat{I}}\right)^{n}>0 .
$$

From the above considerations it follows that:

- the vector

$$
\psi\rangle_{\mathbf{P}+\Delta \mathbf{P}}^{|\Delta \mathbf{P}|^{\frac{1}{4}}}:=\frac{-\frac{1}{2 \pi i} \oint \frac{1}{H_{\mathbf{P}+\Delta \mathbf{P},|\Delta \mathbf{P}|^{\frac{1}{4}}}-E} d E \psi_{\mathbf{P}}^{|\Delta \mathbf{P}|^{\frac{1}{4}}}}{\left\|-\frac{1}{2 \pi i} \oint_{\frac{1}{H_{\mathbf{P}+\Delta \mathbf{P},|\Delta \mathbf{P}|^{\frac{1}{4}}}-E}} d E \psi_{\mathbf{P}}^{|\Delta \mathbf{P}|^{\frac{1}{4}} \|}\right\|}
$$

is ground state of $H_{\mathbf{P}+\Delta \mathbf{P},|\Delta \mathbf{P}|^{\frac{1}{4}}}$

- the following estimate holds

$$
\left\|\psi \gamma_{\mathbf{P}+\Delta \mathbf{P}}^{|\Delta \mathbf{P}|^{\frac{1}{4}}}-\psi_{\mathbf{P}}^{|\Delta \mathbf{P}|^{\frac{1}{4}}}\right\| \leq C^{\prime}|\Delta \mathbf{P}|^{\frac{3}{8}}
$$

where $C^{\prime}$ is a constant uniform in $\mathbf{P}, \mathbf{P}+\Delta \mathbf{P} \in \Sigma$ and $\Delta \mathbf{P} \in \widehat{I}$. Since:

$$
\begin{aligned}
& \text { 1) } \nabla E^{|\Delta \mathbf{P}|^{\frac{1}{4}}}(\mathbf{P}+\Delta \mathbf{P})-\nabla E^{|\Delta \mathbf{P}|^{\frac{1}{4}}}(\mathbf{P}) \\
& =\left(\psi_{\mathbf{P}+\Delta \mathbf{P}}^{\mid \Delta \mathbf{P}}, \frac{{ }^{\frac{1}{4}}}{\mathbf{P}+\Delta \mathbf{P}-\mathbf{P}^{p h}} \psi_{\left.\right|_{\mathbf{P}+\Delta \mathbf{P}} ^{\prime}}^{|\Delta \mathbf{P}|^{\frac{1}{4}}}\right)-\left(\psi_{\mathbf{P}}^{|\Delta \mathbf{P}|^{\frac{1}{4}}}, \frac{\mathbf{P}-\mathbf{P}^{p h}}{m} \psi_{\mathbf{P}}^{|\Delta \mathbf{P}|^{\frac{1}{4}}}\right) \\
& \text { 2) } H_{\mathbf{P},|\Delta \mathbf{P}|^{\frac{1}{4}}}+2 \pi g^{2} \kappa-\frac{\left(\mathbf{P}^{p h}-\mathbf{P}\right)^{2}}{2 m} \geq 0
\end{aligned}
$$


we can conclude that

$$
\left|\nabla E^{|\Delta \mathbf{P}|^{\frac{1}{4}}}(\mathbf{P})-\nabla E^{|\Delta \mathbf{P}|^{\frac{1}{4}}}(\mathbf{P}+\Delta \mathbf{P})\right| \leq C^{\prime \prime}|\Delta \mathbf{P}|^{\frac{3}{8}}
$$

where $C^{\prime \prime}$ is a constant uniform in $\mathbf{P}, \mathbf{P}+\Delta \mathbf{P} \in \Sigma$ and $\Delta \mathbf{P} \in \widehat{I}$.

Turning to the expression to be proved, if $\sigma<m^{\frac{3}{4}}|\Delta \mathbf{P}|^{\frac{1}{4}}$ we take advantage of the result of Lemma A2 (in Appendix) together with Theorem 2.3, which implies that

$$
\left|\nabla E^{|\Delta \mathbf{P}|^{\frac{1}{4}}}(\mathbf{P})-\nabla E^{\sigma}(\mathbf{P})\right|
$$

is of order $|\Delta \mathbf{P}|^{\frac{1}{16}}$, for $\mathbf{P} \in \Sigma$, at most. If $\sigma \geq m^{\frac{3}{4}}|\Delta \mathbf{P}|^{\frac{1}{4}}$, an estimate analogous to (14) holds.

Under the same hypotheses of Lemma 3.3, we have an analogous result about the regularity of the ground eigenvectors in the variable $\mathbf{P}$.

Theorem 3.4 Under the constructive hypotheses, for $\frac{k}{m}$ and $g$ sufficiently small, the norm difference between $\phi_{\mathbf{P}}^{\sigma}$ and $\phi_{\mathbf{P}+\Delta \mathbf{P}}^{\sigma}$ is Hölder in $|\Delta \mathbf{P}|$ with coefficient $\frac{1}{16}-\delta, \delta>0$ and arbitrarily small. The multiplicative constant, $C_{\delta}$, is uniform in $0 \leq \sigma<\kappa \epsilon$, in $\mathbf{P}, \mathbf{P}+\Delta \mathbf{P} \in \Sigma$ and $\Delta \mathbf{P} \in I, I$ a sufficiently small fixed ball around $\Delta \mathbf{P}=0$.

Proof. Preliminary computation:

$$
\begin{aligned}
& H_{\mathbf{P}+\Delta \mathbf{P},|\Delta \mathbf{P}|^{\frac{1}{4}}-H^{w}}^{w},|\Delta \mathbf{P}|^{\frac{1}{4}} \\
& =c_{\mathbf{P}+\Delta \mathbf{P}}^{|\Delta \mathbf{P}|^{\frac{1}{4}}}-c_{\mathbf{P}}^{|\Delta \mathbf{P}|^{\frac{1}{4}}}+\int\left(\mathbf{k} \cdot \nabla E_{\mathbf{P}}^{|\Delta \mathbf{P}|^{\frac{1}{4}}}-\mathbf{k} \cdot \nabla E_{\mathbf{P}+\Delta \mathbf{P}}^{|\Delta \mathbf{P}|^{\frac{1}{4}}}\right) b^{\dagger}(\mathbf{k}) b(\mathbf{k}) d^{3} k \\
& +\frac{1}{2 m}\left(g \int_{m^{\frac{3}{4}}|\Delta \mathbf{P}|^{\frac{1}{4}}}^{\kappa} \frac{\mathbf{k}\left(\widehat{\mathbf{k}} \cdot \nabla E_{\mathbf{P}}^{|\Delta \mathbf{P}|^{\frac{1}{4}}}-\widehat{\mathbf{k}} \cdot \nabla E_{\mathbf{P}+\Delta \mathbf{P}}^{\mid \Delta \mathbf{P}}\right)\left(b(\mathbf{k})+b^{\dagger}(\mathbf{k})\right)}{\sqrt{2}|\mathbf{k}|^{\frac{3}{2}}\left(1-\widehat{\mathbf{k}} \cdot \nabla E_{\mathbf{P}+\Delta \mathbf{P}}^{|\Delta \mathbf{P}|^{\frac{1}{4}}}\right)\left(1-\widehat{\mathbf{k}} \cdot \nabla E_{\mathbf{P}}^{|\Delta \mathbf{P}|^{\frac{1}{4}}}\right)} d^{3} k+\Delta \Pi_{\mathbf{P},|\Delta \mathbf{P}|^{\frac{1}{4}}}\right)^{2} \\
& +\frac{\Gamma}{2 m} \cdot\left(g \int_{m^{\frac{3}{4}}|\Delta \mathbf{P}|^{\frac{1}{4}}}^{\kappa} \frac{\mathbf{k}\left(\widehat{\mathbf{k}} \cdot \nabla E_{\mathbf{P}}^{|\Delta \mathbf{P}|^{\frac{1}{4}}}-\widehat{\mathbf{k}} \cdot \nabla E_{\mathbf{P}+\Delta \mathbf{P}}^{\mid \Delta \mathbf{P}}\right)\left(b(\mathbf{k})+b^{\dagger}(\mathbf{k})\right)}{\sqrt{2}|\mathbf{k}|^{\frac{3}{2}}\left(1-\widehat{\mathbf{k}} \cdot \nabla E_{\mathbf{P}+\Delta \mathbf{P}}^{|\Delta \mathbf{P}|^{\frac{1}{4}}}\right)\left(1-\widehat{\mathbf{k}} \cdot \nabla E_{\mathbf{P}}^{|\Delta \mathbf{P}|^{\frac{1}{4}}}\right)} d^{3} k+\Delta \Pi_{\mathbf{P},|\Delta \mathbf{P}|^{\frac{1}{4}}}\right)
\end{aligned}
$$

where the additive constant $c_{\mathbf{P}}^{|\Delta \mathbf{P}|^{\frac{1}{4}}}$ corresponds to the infrared cutoff $\sigma=m^{\frac{3}{4}}|\Delta \mathbf{P}|^{\frac{1}{4}}$, $\nabla E_{\mathbf{P}}^{|\Delta \mathbf{P}|^{\frac{1}{4}}}$ is a short notation for $\left.\nabla E^{\sigma}(\mathbf{P})\right|_{\sigma=m^{\frac{3}{4}}|\Delta \mathbf{P}|^{\frac{1}{4}}}$ and

$$
\Delta \Pi_{\mathbf{P},|\Delta \mathbf{P}|^{\frac{1}{4}}}=\left(\phi_{\mathbf{P}}^{|\Delta \mathbf{P}|^{\frac{1}{4}}}, \Pi_{\mathbf{P},|\Delta \mathbf{P}|^{\frac{1}{4}}} \phi_{\mathbf{P}}^{|\Delta \mathbf{P}|^{\frac{1}{4}}}\right)-\left(\phi_{\mathbf{P}+\Delta \mathbf{P}}^{|\Delta \mathbf{P}|^{\frac{1}{4}}}, \Pi_{\mathbf{P}+\Delta \mathbf{P},|\Delta \mathbf{P}|^{\frac{1}{4}}} \phi_{\mathbf{P}+\Delta \mathbf{P}}^{|\Delta \mathbf{P}|^{\frac{1}{4}}}\right) .
$$


Considering that for $\mathbf{P}, \mathbf{P}+\Delta \mathbf{P} \in \Sigma$ and $\Delta \mathbf{P} \in \widehat{I}$

- the estimate (14), in Lemma 3.3, holds:

$$
\left|\nabla E^{|\Delta \mathbf{P}|^{\frac{1}{4}}}(\mathbf{P})-\nabla E^{|\Delta \mathbf{P}|^{\frac{1}{4}}}(\mathbf{P}+\Delta \mathbf{P})\right| \leq C^{\prime \prime}|\Delta \mathbf{P}|^{\frac{3}{8}}
$$

- $\Delta \Pi_{\mathbf{P},|\Delta \mathbf{P}|^{\frac{1}{4}}}^{i}$ is estimated of order $|\Delta \mathbf{P}|^{\frac{3}{8}}$ (see equation (6), paragraph 2.1)

- the operator

$$
\frac{H_{\mathbf{P}+\Delta \mathbf{P},|\Delta \mathbf{P}|^{\frac{1}{4}}}^{w}-H_{\mathbf{P},|\Delta \mathbf{P}|^{\frac{1}{4}}}^{w}}{m^{-\frac{3}{8}}|\Delta \mathbf{P}|^{\frac{3}{8}}}
$$

is relatively form-bounded with respect to $H_{\mathbf{P},|\Delta \mathbf{P}|^{\frac{1}{4}}}^{w}$, uniformly in $|\Delta \mathbf{P}|$;

- the gap of $E_{\mathbf{P}}^{|\Delta \mathbf{P}|^{\frac{1}{4}}}$ (as ground energy of $\left.H_{\mathbf{P},|\Delta \mathbf{P}|^{\frac{1}{4}}}^{w}\right|_{F_{|\Delta|^{+}}}$) is bounded from below by $\frac{m^{\frac{3}{4}}|\Delta \mathbf{P}|^{\frac{1}{4}}}{2}$

we conclude that the vector

$$
\phi_{\mathbf{P}+\Delta \mathbf{P}}^{|\Delta \mathbf{P}|^{\frac{1}{4}}}=\frac{-\frac{1}{2 \pi i} \oint \frac{1}{H_{\mathbf{P}+\Delta \mathbf{P},|\Delta \mathbf{P}|^{\frac{1}{4}}}^{-E}} d E \psi_{0}}{\left\|-\frac{1}{2 \pi i} \oint \frac{1}{H_{\mathbf{P}+\Delta \mathbf{P},|\Delta \mathbf{P}|^{\frac{1}{4}}}^{w}} d E \psi_{0}\right\|}
$$

(where $E \in \mathcal{C}$ and s.t. $\left|E-E_{\mathbf{P}+\Delta \mathbf{P} \mid}^{|\Delta \mathbf{P}|^{\frac{1}{4}}}\right|=\frac{m^{\frac{3}{4}}|\Delta \mathbf{P}|^{\frac{1}{4}}}{4}$ ) can be obtained perturbing $\phi_{\mathbf{P}}^{|\Delta \mathbf{P}|^{\frac{1}{4}}}$ for $\Delta \mathbf{P} \in I \subset \widehat{I}, I$ a sufficiently small ball around $\Delta \mathbf{P}=0$.

From the perturbation we also get the estimate:

$$
\left\|\phi_{\mathbf{P}+\Delta \mathbf{P}}^{\mid \Delta \mathbf{P}}-\left.\phi_{\mathbf{P}}^{\mid \frac{1}{4}}\right|^{\frac{1}{4}}\right\| \leq C^{\prime \prime \prime}|\Delta \mathbf{P}|^{\frac{1}{16}}
$$

where the constant $C^{\prime \prime \prime}$ is uniform in $\mathbf{P}, \mathbf{P}+\Delta \mathbf{P} \in \Sigma$ and $\Delta \mathbf{P} \in I$.

For $\sigma<m^{\frac{3}{4}}|\Delta \mathbf{P}|^{\frac{1}{4}}$, the thesis is proved by the norm inequality below

$$
\left\|\phi_{\mathbf{P}+\Delta \mathbf{P}}^{\sigma}-\phi_{\mathbf{P}}^{\sigma}\right\| \leq\left\|\phi_{\mathbf{P}+\Delta \mathbf{P}}^{\sigma}-\phi_{\mathbf{P}+\left.\Delta \mathbf{P}\right|^{\frac{1}{4}}}^{\mid \Delta \mathbf{P}^{2}}\right\|+\left\|\phi_{\mathbf{P}+\Delta \mathbf{P}}^{\mid \Delta \mathbf{P}}-\phi_{\mathbf{P}}^{|\Delta \mathbf{P}|^{\frac{1}{4}}}\right\|+\left\|\phi_{\mathbf{P}}^{|\Delta \mathbf{P}|^{\frac{1}{4}}}-\phi_{\mathbf{P}}^{\sigma}\right\|
$$

and using Theorem 3.2.

If $\sigma \geq m^{\frac{3}{4}}|\Delta \mathbf{P}|^{\frac{1}{4}}$, an estimate analogous to (15) holds. 


\section{Appendix}

Preliminary remark on Lemma A1

Analogously to Lemma 1.3, we want to prove that, for $\mathbf{P} \in \Sigma$ and $g$ sufficiently small, the operator

$$
\left(\Delta H_{\mathbf{P}}^{w}\right)_{\sigma_{j}+1}^{\sigma_{j}}:=\widehat{H}_{\mathbf{P}, \sigma_{j+1}}^{w}-\widehat{c}_{\mathbf{P}}^{\sigma_{j+1}}+c_{\mathbf{P}}^{\sigma_{j}}-H_{\mathbf{P}, \sigma_{j}}^{w}
$$

is small with respect to $\left.H_{\mathbf{P}, \sigma_{j}}^{w}\right|_{F_{\sigma_{j+1}}^{+}}$in a generalized sense. We aim at expanding the resolvent

$$
\left.\frac{1}{\widehat{H}_{\mathbf{P}, \sigma_{j+1}}^{w}-\left(E_{j+1}+\widehat{c}_{\mathbf{P}}^{\sigma_{j+1}}-c_{\mathbf{P}}^{\sigma_{j}}\right)}\right|_{F_{\sigma_{j+1}}^{+}}
$$

(where $E_{j+1} \in \mathcal{C}$ s.t. $\left|E_{j+1}-E_{\mathbf{P}}^{\sigma_{j}}\right|=\frac{11 \sigma_{j+1}}{20}$, and $\widehat{c}_{\mathbf{P}}^{\sigma_{j+1}}-c_{\mathbf{P}}^{\sigma_{j}}=-g^{2} \int_{\sigma_{j+1}}^{\sigma_{j}} \frac{1}{2|\mathbf{k}|^{2} \alpha_{\mathbf{P}}^{\sigma_{j}}(\widehat{\mathbf{k}})} d^{3} k$ ) in terms of $\left.\frac{1}{H_{\mathbf{P}, \sigma_{j}}^{w}-E_{j+1}}\right|_{F_{\sigma_{j+1}}^{+}}$and $\left(\Delta H_{\mathbf{P}}^{w}\right)_{\sigma_{j+1}}^{\sigma_{j}}$.

Lemma A1 Given the spectral properties pointed out in paragraph 2.1, for suffciently small $g$ and for $\mathbf{P} \in \Sigma,\left(\Delta H_{\mathbf{P}}^{w}\right)_{\sigma_{j+1}}^{\sigma_{j}}$ is small with respect to $H_{\mathbf{P}, \sigma_{j}}^{w}$ in the following sense:

given $E_{j+1} \in \mathcal{C}$ s.t. $\left|E_{j+1}-E_{\mathbf{P}}^{\sigma_{j}}\right|=\frac{11}{20} \sigma_{j+1}$,

$$
\left\|\frac{1}{H_{\mathbf{P}, \sigma_{j}}^{w}-E_{j+1}}\left(-\left(\Delta H_{\mathbf{P}}^{w}\right)_{\sigma_{j+1}}^{\sigma_{j}} \frac{1}{H_{\mathbf{P}, \sigma_{j}}^{w}-E_{j+1}}\right)^{n}\right\|_{F_{\sigma_{j+1}}^{+}} \leq \frac{20\left(C_{g}\right)^{n}}{\sigma_{j+1}}
$$

where $0<C_{g}<\frac{1}{12}$; therefore the series expansion below is well defined:

$$
\begin{aligned}
& \left.\frac{1}{\widehat{H}_{\mathbf{P}, \sigma_{j+1}}^{w}-\left(E_{j+1}+\widehat{c}_{\mathbf{P}}^{\sigma_{j+1}}-c_{\mathbf{P}}^{\sigma_{j}}\right)}\right|_{F_{\sigma_{j+1}}^{+}} \\
& =\left.\frac{1}{H_{\mathbf{P}, \sigma_{j}}^{w}+\left(\Delta H_{\mathbf{P}}^{w}\right)_{\sigma_{j+1}}^{\sigma_{j}}-c_{\mathbf{P}}^{\sigma_{j}}+\widehat{c}_{\mathbf{P}}^{\sigma_{j+1}}-\left(E_{j+1}-c_{\mathbf{P}}^{\sigma_{j}}+\widehat{c}_{\mathbf{P}}^{\sigma_{j+1}}\right)}\right|_{F_{\sigma_{j+1}}^{+}} \\
& =\left.\frac{1}{H_{\mathbf{P}, \sigma_{j}}^{w}-E_{j+1}}\right|_{F_{\sigma_{j+1}}^{+}}+\left.\frac{1}{H_{\mathbf{P}, \sigma_{j}}^{w}-E_{j+1}} \sum_{n=1}^{+\infty}\left(-\left(\Delta H_{\mathbf{P}}^{w}\right)_{\sigma_{j+1}}^{\sigma_{j}} \frac{1}{H_{\mathbf{P}, \sigma_{j}}^{w}-E_{j+1}}\right)^{n}\right|_{F_{\sigma_{j+1}}^{+}} .
\end{aligned}
$$

Proof. Following the proof of Lemma 1.3, we discuss the norm of

$$
\left.\left(\frac{1}{H_{\mathbf{P}, \sigma_{j}}^{w}-E_{j+1}}\right)^{\frac{1}{2}}\left(\Delta H_{\mathbf{P}}^{w}\right)_{\sigma_{j+1}}^{\sigma_{j}}\left(\frac{1}{H_{\mathbf{P}, \sigma_{j}}^{w}-E_{j+1}}\right)^{\frac{1}{2}}\right|_{F_{\sigma_{j+1}}^{+}}
$$


where $\left(\Delta H_{\mathbf{P}}^{w}\right)_{\sigma_{j+1}}^{\sigma_{j}}=\left[\left(\Delta H_{\mathbf{P}}^{w}\right)_{\sigma_{j+1}}^{\sigma_{j}}\right]^{m i x}+\left[\left(\Delta H_{\mathbf{P}}^{w}\right)_{\sigma_{j+1}}^{\sigma_{j}}\right]^{\text {quad. }}$ with

$$
\begin{aligned}
& {\left[\left(\Delta H_{\mathbf{P}}^{w}\right)_{\sigma_{j+1}}^{\sigma_{j}}\right]^{\text {quad. }}:=} \\
& =\frac{1}{2 m}\left(g \int_{\sigma_{j+1}}^{\sigma_{j}} \frac{\mathbf{k}\left(b(\mathbf{k})+b^{\dagger}(\mathbf{k})\right)}{\sqrt{2}|\mathbf{k}|^{\frac{3}{2}} \alpha_{\mathbf{P}}^{\sigma_{j}}(\mathbf{k})} d^{3} k-g^{2} \int_{\sigma_{j+1}}^{\sigma_{j}} \frac{\mathbf{k}}{2|\mathbf{k}|^{3}\left(\alpha_{\mathbf{P}}^{\sigma_{j}}(\mathbf{k})\right)^{2}} d^{3} k\right)^{2} \\
& {\left[\left(\Delta H_{\mathbf{P}}^{w}\right)_{\sigma_{j+1}}^{\sigma_{j}}\right]^{m i x}:=} \\
& =\frac{1}{2 m}\left\{\Gamma_{\mathbf{P}, \sigma_{j}} \cdot\left(-g \int_{\sigma_{j+1}}^{\sigma_{j}} \frac{\mathbf{k}\left(b(\mathbf{k})+b^{\dagger}(\mathbf{k})\right)}{\sqrt{2}|\mathbf{k}|^{\frac{3}{2}} \alpha_{\mathbf{P}}^{\sigma_{j}}(\mathbf{k})} d^{3} k+g^{2} \int_{\sigma_{j+1}}^{\sigma_{j}} \frac{\mathbf{k}}{2|\mathbf{k}|^{3}\left(\alpha_{\mathbf{P}}^{\sigma_{j}}(\mathbf{k})\right)^{2}} d^{3} k\right)+h . c .\right\} .
\end{aligned}
$$

In order to control the above quantities, we use the following estimate again and again

$$
\left\|\int_{\sigma_{j+1}}^{\sigma_{j}} k^{i} b(\mathbf{k}) \frac{d^{3} k}{\sqrt{2}|\mathbf{k}|^{\frac{3}{2}} \alpha_{\mathbf{P}}^{\sigma_{j}}(\mathbf{k})}\left(\frac{1}{H_{\mathbf{P}, \sigma_{j}}^{w}-E_{j+1}}\right)^{\frac{1}{2}}\right\|_{F_{\sigma_{j+1}}^{+}} \leq \frac{1}{1-v^{\max }} \sqrt{10 \pi} \cdot \sigma_{j}^{\frac{1}{2}}
$$

which essentially derives from the estimate (1) of Lemma 1.3, by performing a unitary transformation, and from the result in Lemma A2, point 1, which ensures that $0<v^{\max }<1$. So we can provide a bound of order $\frac{\sigma_{j}}{\kappa}$ for the norm of the "quadratic terms":

$\left\|\left(\frac{1}{H_{\mathbf{P}, \sigma_{j}}^{w}-E_{j+1}}\right)^{\frac{1}{2}}\left[\left(\Delta H_{\mathbf{P}}^{w}\right)_{\sigma_{j+1}}^{\sigma_{j}}\right]^{q u a d .}\left(\frac{1}{H_{\mathbf{P}, \sigma_{j}}^{w}-E_{j+1}}\right)^{\frac{1}{2}}\right\|_{F_{\sigma_{j+1}}^{+}} \leq \frac{\sigma_{j}}{\kappa} \cdot C_{1}\left(g, \frac{\kappa}{m}\right)$.

For the mixed terms (a2) containing the operators $\Gamma_{\mathbf{P}, \sigma_{j}}$, we exploit the fact that the norm

$$
\left\|\left(\frac{1}{H_{\mathbf{P}, \sigma_{j}}^{w}-E_{j+1}}\right)^{\frac{1}{2}} \frac{\Gamma_{\mathbf{P}, \sigma_{j}}^{i}}{\sqrt{m}}\right\|_{F_{\sigma_{j+1}}^{+}}=\left\|\frac{\Gamma_{\mathbf{P}, \sigma_{j}}^{i}}{\sqrt{m}}\left[\left(\frac{1}{H_{\mathbf{P}, \sigma_{j}}^{w}-E_{j+1}}\right)^{\frac{1}{2}}\right]^{\dagger}\right\|_{F_{\sigma_{j+1}}^{+}}
$$

is of order $\left(\frac{\kappa}{\sigma_{j+1}}\right)^{\frac{1}{2}}$, which follows from the form inequality

$$
\left(\Gamma_{\mathbf{P}, \sigma_{j}}^{i}\right)^{2} \leq 2 m\left(H_{\mathbf{P}, \sigma_{j}}^{w}-c_{\mathbf{P}}^{\sigma_{j}}\right)
$$

Therefore a uniform bound is worked out for them:

$$
\left\|\left(\frac{1}{H_{\mathbf{P}, \sigma_{j}}^{w}-E_{j+1}}\right)^{\frac{1}{2}}\left[\left(\Delta H_{\mathbf{P}}^{w}\right)_{\sigma_{j+1}}^{\sigma_{j}}\right]^{m i x}\left(\frac{1}{H_{\mathbf{P}, \sigma_{j}}^{w}-E_{j+1}}\right)^{\frac{1}{2}}\right\|_{F_{\sigma_{j+1}}^{+}} \leq C_{2}\left(g, \frac{\kappa}{m}\right) .
$$




\section{Conclusion}

For $g$ sufficiently small, the $j$-independent constants $C_{1,2}$ can be tuned so that the thesis is proved.

Lemma A2 Under the constructive hypotheses the following results for $\nabla E^{\sigma}(\mathbf{P})$ hold:

1) $\left|\nabla E^{\sigma}(\mathbf{P})\right|<v^{\max }<\frac{1}{\sqrt{80}} \quad \forall \sigma ;$

2) for $g$ sufficiently small

$$
\left|\nabla E^{\sigma_{j+1}}(\mathbf{P})-\nabla E^{\sigma_{j}}(\mathbf{P})\right|<C^{\nabla E} \cdot\left(\left\|\frac{\widehat{\phi}_{\mathbf{P}}^{\sigma_{j+1}}}{\left\|\widehat{\phi}_{\mathbf{P}}^{\sigma_{j+1}}\right\|}-\frac{\phi_{\mathbf{P}}^{\sigma_{j}}}{\left\|\phi_{\mathbf{P}}^{\sigma_{j}}\right\|}\right\|+\epsilon^{\frac{j+1}{8}}\right)
$$

where $C^{\nabla E}$ is uniform in $j \in N$.

Proof. 1) Let us start from

$$
\begin{aligned}
\left|\nabla E^{\sigma}(\mathbf{P})\right| & =\frac{\left[\sum_{i}\left(\psi_{\mathbf{P}}^{\sigma},\left(P^{i}-P^{p h^{i}}\right) \psi_{\mathbf{P}}^{\sigma}\right)^{2}\right]^{\frac{1}{2}}}{m\left\|\psi_{\mathbf{P}}^{\sigma}\right\|^{2}} \\
& \leq \sqrt{\frac{2}{m}} \cdot \frac{\left[\left(\psi_{\mathbf{P}}^{\sigma},\left(H_{\mathbf{P}, \sigma}+2 \pi g^{2} \kappa\right) \psi_{\mathbf{P}}^{\sigma}\right)\right]^{\frac{1}{2}}}{\left\|\psi_{\mathbf{P}}^{\sigma}\right\|}
\end{aligned}
$$

According to the constructive hypotheses, Section 1, we have:

- $\frac{\left|\left(\psi_{\mathbf{P}}^{\sigma}, H_{\mathbf{P}, \sigma} \psi_{\mathbf{P}}^{\sigma}\right)\right|}{\left\|\psi_{\mathbf{P}}^{\sigma}\right\|^{2}} \leq\left|\left(\psi_{0}, H_{\mathbf{P}, \sigma} \psi_{0}\right)\right|=\frac{\mathbf{P}^{2}}{2 m} \leq \frac{m}{2 \cdot 400}$

- $\frac{\left|\left(\psi_{\mathbf{P}}^{\sigma}, 2 \pi g^{2} \kappa \psi_{\mathbf{P}}^{\sigma}\right)\right|}{\left\|\psi_{\mathbf{P}}^{\sigma}\right\|^{2}} \leq \frac{m}{2 \cdot 100}$

The upper bound for the absolute value of the gradient, $v^{\max }$, is surely smaller than $\frac{1}{\sqrt{80}}$.

2) Let us analyze the difference between the gradients of the ground energy at subsequent infrared cutoffs. Starting from the relation (6), paragraph 2.1,

$$
m \nabla E_{\mathbf{P}}^{\sigma_{j}}=\mathbf{P}-\frac{\left(\phi_{\mathbf{P}}^{\sigma_{j}}, \Pi_{\mathbf{P}, \sigma_{j}} \phi_{\mathbf{P}}^{\sigma_{j}}\right)}{\left\|\phi_{\mathbf{P}}^{\sigma_{j}}\right\|^{2}}-g^{2} \int_{\sigma_{j}}^{\kappa} \frac{\mathbf{k}}{2|\mathbf{k}|^{3}\left(\alpha_{\mathbf{P}}^{\sigma_{j}}(\widehat{\mathbf{k}})\right)^{2}} d^{3} k,
$$

we obtain

$$
\begin{aligned}
& m \nabla E_{\mathbf{P}}^{\sigma_{j}}-m \nabla E_{\mathbf{P}}^{\sigma_{j+1}}-g^{2} \int_{\sigma_{j+1}}^{\kappa} \frac{\mathbf{k}}{2|\mathbf{k}|^{3}\left(\alpha_{\mathbf{P}}^{\sigma_{j+1}}(\widehat{\mathbf{k}})\right)^{2}} d^{3} k+g^{2} \int_{\sigma_{j}}^{\kappa} \frac{\mathbf{k}}{2|\mathbf{k}|^{3}\left(\alpha_{\mathbf{P}}^{\sigma_{j}}(\widehat{\mathbf{k}})\right)^{2}} d^{3} k \\
& =\frac{1}{\left\|\hat{\phi}_{\mathbf{P}}^{\sigma_{j+1}}\right\|^{2}} \cdot\left(\widehat{\phi}_{\mathbf{P}}^{\sigma_{j+1}}, \widehat{\Pi}_{\mathbf{P}, \sigma_{j+1}} \widehat{\phi}_{\mathbf{P}}^{\sigma_{j+1}}\right)-\frac{1}{\left\|\phi_{\mathbf{P}}^{\sigma_{j}}\right\|^{2}} \cdot\left(\phi_{\mathbf{P}}^{\sigma_{j}}, \Pi_{\mathbf{P}, \sigma_{j}}, \phi_{\mathbf{P}}^{\sigma_{j}}\right) .
\end{aligned}
$$


By simple steps we relate the difference between the two gradients to the norm difference of the corresponding ground eigenvectors:

$$
\begin{array}{rl}
m & m E_{\mathbf{P}}^{\sigma_{j}}-m \nabla E_{\mathbf{P}}^{\sigma_{j+1}}+g^{2} \int_{\sigma_{j}}^{\kappa} \frac{\mathbf{k}\left(-\widehat{\mathbf{k}} \cdot \nabla E_{\mathbf{P}}^{\sigma_{j+1}}+\widehat{\mathbf{k}} \cdot \nabla E_{\mathbf{P}}^{\sigma_{j}}\right)\left(2-\widehat{\mathbf{k}} \cdot \nabla E_{\mathbf{P}}^{\sigma_{j}}-\widehat{\mathbf{k}} \cdot \nabla E_{\mathbf{P}}^{\sigma_{j+1}}\right)}{2|\mathbf{k}|^{3}\left(\alpha_{\mathbf{P}}^{\sigma_{j}+1}(\widehat{\mathbf{k}})\right)^{2}\left(\alpha_{\mathbf{P}}^{\sigma_{j}}(\widehat{\mathbf{k}})\right)^{2}} d^{3} k \\
= & \frac{1}{\left\|\hat{\phi}_{\mathbf{P}}^{\sigma_{j+1}}\right\|} \cdot\left(\widehat{\phi}_{\mathbf{P}}^{\sigma_{j+1}}, \widehat{\Pi}_{\mathbf{P}, \sigma_{j+1}}\left(\frac{\widehat{\phi}_{\mathbf{P}}^{\sigma_{j+1}}}{\left\|\widehat{\phi}_{\mathbf{P}}^{\sigma_{j+1}}\right\|}-\frac{\phi_{\mathbf{P}}^{\sigma_{j}}}{\left\|\phi_{\mathbf{P}}^{\sigma_{j}}\right\|}\right)\right)+g^{2} \int_{\sigma_{j+1}}^{\sigma_{j}} \frac{\mathbf{k}}{2|\mathbf{k}|^{3}\left(\alpha_{\mathbf{P}}^{\sigma_{j+1}}(\widehat{\mathbf{k}})\right)^{2}} d^{3} k \\
& +\frac{1}{\left\|\widehat{\phi}_{\mathbf{P}}^{\sigma_{j+1}}\right\|\left\|\phi_{\mathbf{P}}^{\sigma_{j}}\right\|} \cdot\left(\widehat{\phi}_{\mathbf{P}}^{\sigma_{j+1}}, \widehat{\Pi}_{\mathbf{P}, \sigma_{j+1}} \phi_{\mathbf{P}}^{\sigma_{j}}\right)-\frac{1}{\left\|\hat{\phi}_{\mathbf{P}}^{\sigma_{j+1}}\right\|\left\|\phi_{\mathbf{P}}^{\sigma_{j}}\right\|} \cdot\left(\widehat{\phi}_{\mathbf{P}}^{\sigma_{j+1}}, \Pi_{\mathbf{P}, \sigma_{j}} \phi_{\mathbf{P}}^{\sigma_{j}}\right) \\
& +\frac{1}{\left\|\widehat{\phi}_{\mathbf{P}}^{\sigma_{j+1}}\right\|\left\|\phi_{\mathbf{P}}^{\sigma_{j}}\right\|} \cdot\left(\hat{\phi}_{\mathbf{P}}^{\sigma_{j+1}}, \Pi_{\mathbf{P}, \sigma_{j}} \phi_{\mathbf{P}}^{\sigma_{j}}\right)-\frac{1}{\left\|\phi_{\mathbf{P}}^{\sigma_{j}}\right\|^{2}} \cdot\left(\phi_{\mathbf{P}}^{\sigma_{j}}, \Pi_{\mathbf{P}, \sigma_{j}} \phi_{\mathbf{P}}^{\sigma_{j}}\right) .
\end{array}
$$

Considering that

$$
\begin{aligned}
& \widehat{\Pi}_{\mathbf{P}, \sigma_{j+1}}^{i}-\Pi_{\mathbf{P}, \sigma_{j}}^{i} \\
& =-g \int_{\sigma_{j+1}}^{\sigma_{j}} \frac{k^{i}\left(b(\mathbf{k})+b^{\dagger}(\mathbf{k})\right)}{\sqrt{2}|\mathbf{k}|^{\frac{3}{2}} \alpha_{\mathbf{P}}^{\sigma_{j}}(\widehat{\mathbf{k}})} d^{3} k \\
& \quad+\frac{g^{2}}{2} \int_{\sigma_{j+1}}^{\kappa} \frac{k^{i}\left(-\widehat{\mathbf{k}} \cdot \nabla E_{\mathbf{P}}^{\sigma_{j+1}}+\widehat{\mathbf{k}} \cdot \nabla E_{\mathbf{P}}^{\sigma_{j}}\right)\left(2-\widehat{\mathbf{k}} \cdot \nabla E_{\mathbf{P}}^{\sigma_{j}}-\widehat{\mathbf{k}} \cdot \nabla E_{\mathbf{P}}^{\sigma_{j+1}}\right)}{|\mathbf{k}|^{3}\left(\alpha_{\mathbf{P}}^{\sigma_{j}}(\widehat{\mathbf{k}})\right)^{2}\left(\alpha_{\mathbf{P}}^{\sigma_{j+1}}(\widehat{\mathbf{k}})\right)^{2}} d^{3} k
\end{aligned}
$$

the equation (a3) can be written in the following way

$$
\begin{aligned}
& m \nabla E_{\mathbf{P}}^{\sigma_{j}}-m \nabla E_{\mathbf{P}}^{\sigma_{j+1}}+g^{2} \int_{\sigma_{j}}^{\kappa} \frac{\mathbf{k}\left(-\widehat{\mathbf{k}} \cdot \nabla E_{\mathbf{P}}^{\sigma_{j+1}}+\widehat{\mathbf{k}} \cdot \nabla E_{\mathbf{P}}^{\sigma_{j}}\right)\left(2-\widehat{\mathbf{k}} \cdot \nabla E_{\mathbf{P}}^{\sigma_{j}}-\widehat{\mathbf{k}} \cdot \nabla E_{\mathbf{P}}^{\sigma_{j+1}}\right)}{2|\mathbf{k}|^{3}\left(\alpha_{\mathbf{P}}^{\sigma_{j+1}}(\widehat{\mathbf{k}})\right)^{2}\left(\alpha_{\mathbf{P}}^{\sigma_{j}}(\widehat{\mathbf{k}})\right)^{2}} d^{3} k \\
& -\frac{1}{\left\|\widehat{\phi}_{\mathbf{P}}^{\sigma_{j+1}}\right\| \cdot\left\|\phi_{\mathbf{P}}^{\sigma_{j}}\right\|} \cdot\left(\widehat{\phi}_{\mathbf{P}}^{\sigma_{j+1}}, \phi_{\mathbf{P}}^{\sigma_{j}}\right) \cdot g^{2} \int_{\sigma_{j+1}}^{\kappa} \frac{\mathbf{k}\left(-\widehat{\mathbf{k}} \cdot \nabla E_{\mathbf{P}}^{\sigma_{j+1}}+\widehat{\mathbf{k}} \cdot \nabla E_{\mathbf{P}}^{\sigma_{j}}\right)\left(2-\widehat{\mathbf{k}} \cdot \nabla E_{\mathbf{P}}^{\sigma_{j}}-\widehat{\mathbf{k}} \cdot \nabla E_{\mathbf{P}}^{\sigma_{j+1}}\right)}{2|\mathbf{k}|^{3}\left(\alpha_{\mathbf{P}}^{\sigma_{j+1}}(\widehat{\mathbf{k}})\right)^{2}\left(\alpha_{\mathbf{P}}^{\sigma_{j}}(\widehat{\mathbf{k}})\right)^{2}} d^{3} k \\
& =\left(\frac{\widehat{\phi}_{\mathbf{P}}^{\sigma_{j+1}}}{\left\|\widehat{\phi}_{\mathbf{P}}^{\sigma_{j+1}}\right\|}, \widehat{\Pi}_{\mathbf{P}, \sigma_{j+1}}\left(\frac{\widehat{\phi}_{\mathbf{P}}^{\sigma_{j+1}}}{\left\|\widehat{\phi}_{\mathbf{P}}^{\sigma_{j+1}}\right\|}-\frac{\phi_{\mathbf{P}}^{\sigma_{j}}}{\left\|\phi_{\mathbf{P}}^{\sigma_{j}}\right\|}\right)\right)+\left(\left(\frac{\widehat{\phi}_{\mathbf{P}}^{\sigma_{j+1}}}{\left\|\widehat{\phi}_{\mathbf{P}}^{\sigma_{j+1}}\right\|}-\frac{\phi_{\mathbf{P}}^{\sigma_{j}}}{\left\|\phi_{\mathbf{P}}^{\sigma_{j}}\right\|}\right), \Pi_{\mathbf{P}, \sigma_{j}} \frac{\phi_{\mathbf{P}}^{\sigma_{j}}}{\left\|\phi_{\mathbf{P}}^{\sigma_{j}}\right\|}\right) \\
& +g^{2} \int_{\sigma_{j+1} \sigma_{j}}^{\sigma_{j}|\mathbf{k}|^{3}\left(\alpha_{\mathbf{P}}^{\sigma_{j+1}}(\widehat{\mathbf{k}})\right)^{2}} d^{3} k-\frac{1}{\left\|\widehat{\phi}_{\mathbf{P}}^{\sigma_{j+1}}\right\| \cdot\left\|\phi_{\mathbf{P}}^{\sigma_{j}}\right\|} \cdot\left(\widehat{\phi}_{\mathbf{P}}^{\sigma_{j+1}}, g \int_{\sigma_{j+1}}^{\sigma_{j}} \frac{\mathbf{k}\left(b(\mathbf{k})+b^{\dagger}(\mathbf{k})\right)}{\sqrt{2}|\mathbf{k}|^{\frac{3}{2}} \alpha_{\mathbf{P}}^{\sigma_{j}}(\widehat{\mathbf{k}})} d^{3} k \phi_{\mathbf{P}}^{\sigma_{j}}\right) .
\end{aligned}
$$

Then the thesis follows from the expression above and from the following considerations.

i) On the left-hand side of the equation there is a quantity whose absolute value is larger than

$$
C\left|\nabla E_{\mathbf{P}}^{\sigma_{j}}-\nabla E_{\mathbf{P}}^{\sigma_{j+1}}\right|
$$

for $g$ sufficiently small, where $C$ is a positive constant that is uniform in $j$ and converges to $m$ for $g \rightarrow 0$. It is due to the result in point 1 ). 
ii) On the right-hand side there is a quantity whose absolute value is bounded by a $g$-dependent, uniform in $j$, constant times the factor

$$
\left(\left\|\frac{\widehat{\phi}_{\mathbf{P}}^{\sigma_{j+1}}}{\left\|\widehat{\phi}_{\mathbf{P}}^{\sigma_{j+1}}\right\|}-\frac{\phi_{\mathbf{P}}^{\sigma_{j}}}{\left\|\phi_{\mathbf{P}}^{\sigma_{j}}\right\|}\right\|+\epsilon^{\frac{j+1}{8}}\right) ;
$$

it is due to the bounds below, where (first estimate) we exploit the form inequality

$$
\int|\mathbf{k}| \alpha_{\mathbf{P}}^{\sigma_{j}}(\widehat{\mathbf{k}}) b^{\dagger}(\mathbf{k}) b(\mathbf{k}) d^{3} k \leq \widehat{H}_{\mathbf{P}, \sigma_{j+1}}^{w}-\widehat{c}_{\mathbf{P}}^{\sigma_{j+1}}
$$

and (second estimate) we use the known unitary transformations to switch from the "Пึ" operators to the " $\Pi$ " ones and finally to $\mathbf{P}^{p h}$ :

$$
\begin{aligned}
\text { - } & \left(\widehat{\phi}_{\mathbf{P}}^{\sigma_{j+1}}, g \int_{\sigma_{j+1}}^{\sigma_{j}} \frac{k^{i}\left(b(\mathbf{k})+b^{\dagger}(\mathbf{k})\right)}{\sqrt{2}|\mathbf{k}|^{\frac{3}{2}} \alpha_{\mathbf{P}}^{\sigma_{j}}(\widehat{\mathbf{k}})} d^{3} k \phi_{\mathbf{P}}^{\sigma_{j}}\right)=\left(\widehat{\phi}_{\mathbf{P}}^{\sigma_{j+1}}, g \int_{\sigma_{j+1}}^{\sigma_{j}} \frac{k^{i} b^{\dagger}(\mathbf{k})}{\sqrt{2}|\mathbf{k}|^{\frac{3}{2}} \alpha_{\mathbf{P}}^{\sigma_{j}(\widehat{\mathbf{k}})}} d^{3} k \phi_{\mathbf{P}}^{\sigma_{j}}\right) \\
= & g \int_{\sigma_{j+1}}^{\sigma_{j}} \frac{k^{i}}{\sqrt{2}|\mathbf{k}|^{2}\left(\alpha_{\mathbf{P}}^{\sigma_{j}}(\widehat{\mathbf{k}})\right)^{\frac{3}{2}}}\left(\widehat{\phi}_{\mathbf{P}}^{\sigma_{j+1}},|\mathbf{k}|^{\frac{1}{2}}\left(\alpha_{\mathbf{P}}^{\sigma_{j}}(\widehat{\mathbf{k}})\right)^{\frac{1}{2}} b^{\dagger}(\mathbf{k}) \phi_{\mathbf{P}}^{\sigma_{j}}\right) d^{3} k \\
& \leq g\left(\int_{\sigma_{j+1}}^{\sigma_{j}} \frac{\left(k^{i}\right)^{2}}{2|\mathbf{k}|^{4}\left(\alpha_{\mathbf{P}}^{\sigma_{j}}(\widehat{\mathbf{k}})\right)^{3}} d^{3} k\right)^{\frac{1}{2}} \cdot\left(\widehat{\phi}_{\mathbf{P}}^{\sigma_{j+1}}, \int|\mathbf{k}| \alpha_{\mathbf{P}}^{\sigma_{j}}(\widehat{\mathbf{k}}) b^{\dagger}(\mathbf{k}) b(\mathbf{k}) d^{3} k \widehat{\phi}_{\mathbf{P}}^{\sigma_{j+1}}\right)^{\frac{1}{2}} \cdot\left\|\phi_{\mathbf{P}}^{\sigma_{j}}\right\| \\
& \leq g\left(\int_{\sigma_{j+1}}^{\sigma_{j}} \frac{\left(k^{i}\right)^{2}}{2|\mathbf{k}|^{4}\left(\alpha_{\mathbf{P}}^{\sigma_{j}}(\widehat{\mathbf{k}})\right)^{3}} d^{3} k\right)^{\frac{1}{2}} \cdot\left(E_{\mathbf{P}}^{\sigma_{j+1}}-\widehat{c}_{\mathbf{P}}(j+1)\right)^{\frac{1}{2}} \cdot\left(\widehat{\phi}_{\mathbf{P}}^{\sigma_{j+1}}, \widehat{\phi}_{\mathbf{P}}^{\sigma_{j+1}}\right)^{\frac{1}{2}} \cdot\left\|\phi_{\mathbf{P}}^{\sigma_{j}}\right\| \\
& \left\|\widehat{\Pi}_{\mathbf{P}, \sigma_{j+1}}^{i} \widehat{\phi}_{\mathbf{P}}^{\sigma_{j+1}}\right\|=\left\|\Pi_{\mathbf{P}, \sigma_{j+1}}^{i} \phi_{\mathbf{P}}^{\sigma_{j+1}}\right\| \leq\left\|\left(P^{p h^{i}}-P^{i}\right) \psi_{\mathbf{P}}^{\sigma_{j+1}}\right\|+b \cdot\left\|\psi_{\mathbf{P}}^{\sigma_{j+1}}\right\| \\
& \leq \sqrt{2 m} \cdot\left(2 \pi g^{2} \kappa+E_{\mathbf{P}}^{\sigma_{j+1}}\right)^{\frac{1}{2}} \cdot\left\|\psi_{\mathbf{P}}^{\sigma_{j+1}}\right\|+b \cdot\left\|\psi_{\mathbf{P}}^{\sigma_{j+1}}\right\|
\end{aligned}
$$

where $b$ is a constant uniform in $j$ and $\mathbf{P} \in \Sigma$.

Lemma A3 For a sufficiently small ratio $\frac{\kappa}{m}$, in addition to the constructive hypotheses, the following inequality holds:

$$
\begin{aligned}
& \left\|\left(\frac{1}{H_{\mathbf{P}, \sigma_{j}}^{w}-E_{j+1}}\right)^{\frac{1}{2}} \int_{\sigma_{j+1}}^{\sigma_{j}} \frac{k^{i} b^{\dagger}(\mathbf{k})}{\sqrt{2}|\mathbf{k}|^{\frac{3}{2}} \alpha_{\mathbf{P}}^{\sigma_{j}}(\widehat{\mathbf{k}})} d^{3} k \Gamma_{\mathbf{P}, \sigma_{j}}^{i}\left(\frac{1}{H_{\mathbf{P}, \sigma_{j}}^{w}-E_{j+1}}\right)^{\frac{1}{2}} \phi_{\mathbf{P}}^{\sigma_{j}}\right\|^{2} \\
& \leq 2 Q(\epsilon) \cdot \sqrt{122} \cdot Z_{\sigma_{j+1}}^{\sigma_{j}} \cdot\left|\frac{1}{E_{\mathbf{P}}^{\sigma_{j}}-E_{j+1}}\right| \cdot\left|\left(\Gamma_{\mathbf{P}, \sigma_{j}}^{i} \phi_{\mathbf{P}}^{\sigma_{j}},\left(\frac{1}{H_{\mathbf{P}, \sigma_{j}}^{w}-E_{j+1}}\right) \Gamma_{\mathbf{P}, \sigma_{j}}^{i} \phi_{\mathbf{P}}^{\sigma_{j}}\right)\right|
\end{aligned}
$$

being $Z_{\sigma_{j+1}}^{\sigma_{j}}=\left\{\sum_{i} \int_{\sigma_{j+1}}^{\sigma_{j}} \frac{k^{i^{2}}}{2|\mathbf{k}|^{3} \alpha_{\mathbf{P}}^{\sigma_{j}}(\widehat{\mathbf{k}})^{2}} d^{3} k\right\}$.

Proof. Let us start from

$$
\begin{aligned}
& \left\|\left(\frac{1}{H_{\mathbf{P}, \sigma_{j}}^{w}-E_{j+1}}\right)^{\frac{1}{2}} \int_{\sigma_{j+1}}^{\sigma_{j}} \frac{k^{i} b^{\dagger}(\mathbf{k})}{\sqrt{2}|\mathbf{k}|^{\frac{3}{2}} \alpha_{\mathbf{P}}^{\sigma_{j}}(\widehat{\mathbf{k}})} d^{3} k \Gamma_{\mathbf{P}, \sigma_{j}}^{i} \phi_{\mathbf{P}}^{\sigma_{j}}\right\|^{2}= \\
& =\left(\int_{\sigma_{j+1}}^{\sigma_{j}} \frac{k^{i} b^{\dagger}(\mathbf{k})}{\sqrt{2}|\mathbf{k}|^{\frac{3}{2}} \alpha_{\mathbf{P}}^{\sigma_{j}}(\widehat{\mathbf{k}})} d^{3} k \Gamma_{\mathbf{P}, \sigma_{j}}^{i} \phi_{\mathbf{P}}^{\sigma_{j}},\left|\frac{1}{H_{\mathbf{P}, \sigma_{j}}^{w}-E_{j+1}}\right| \int_{\sigma_{j+1}}^{\sigma_{j}} \frac{k^{i} b^{\dagger}(\mathbf{k})}{\sqrt{2}|\mathbf{k}|^{\frac{3}{2}} \alpha_{\mathbf{P}}^{\sigma_{j}}(\widehat{\mathbf{k}})} d^{3} k \Gamma_{\mathbf{P}, \sigma_{j}}^{i} \phi_{\mathbf{P}}^{\sigma_{j}}\right) .
\end{aligned}
$$


Now, from Lemma 2.1

$\left(\int_{\sigma_{j+1}}^{\sigma_{j}} \frac{k^{i} b^{\dagger}(\mathbf{k})}{\sqrt{2}|\mathbf{k}|^{\frac{3}{2}} \alpha_{\mathbf{P}}^{\sigma_{j}(\widehat{\mathbf{k}})}} d^{3} k \Gamma_{\mathbf{P}, \sigma_{j}}^{i} \phi_{\mathbf{P}}^{\sigma_{j}},\left|\frac{1}{H_{\mathbf{P}, \sigma_{j}}^{w}-E_{j+1}}\right| \int_{\sigma_{j+1}}^{\sigma_{j}} \frac{k^{i} b^{\dagger}(\mathbf{k})}{\sqrt{2}|\mathbf{k}|^{\frac{3}{2}} \alpha_{\mathbf{P}}^{\sigma_{j}}(\widehat{\mathbf{k}})} d^{3} k \Gamma_{\mathbf{P}, \sigma_{j}}^{i} \phi_{\mathbf{P}}^{\sigma_{j}}\right)$
$\leq \sqrt{122} \cdot\left|\left(\int_{\sigma_{j+1}}^{\sigma_{j}} \frac{k^{i} b^{\dagger}(\mathbf{k})}{\sqrt{2}|\mathbf{k}|^{\frac{3}{2}} \alpha_{\mathbf{P}}^{\sigma_{j}}(\widehat{\mathbf{k}})} d^{3} k \Gamma_{\mathbf{P}, \sigma_{j}}^{i} \phi_{\mathbf{P}}^{\sigma_{j}},\left(\frac{1}{H_{\mathbf{P}, \sigma_{j}}^{w}-E_{j+1}}\right) \int_{\sigma_{j+1}}^{\sigma_{j}} \frac{k^{i} b^{\dagger}(\mathbf{k})}{\sqrt{2}|\mathbf{k}|^{\frac{3}{2}} \alpha_{\mathbf{P}}^{\sigma_{j}}(\widehat{\mathbf{k}})} d^{3} k \Gamma_{\mathbf{P}, \sigma_{j}}^{i} \phi_{\mathbf{P}}^{\sigma_{j}}\right)\right|$.

Starting from the expression

$$
H_{\mathbf{P}, \sigma_{j}}^{w}=\frac{1}{2 m} \Gamma_{\mathbf{P}, \sigma_{j}}^{2}+\int|\mathbf{k}| \alpha_{\mathbf{P}}^{\sigma_{j}}(\widehat{\mathbf{k}}) b^{\dagger}(\mathbf{k}) b(\mathbf{k}) d^{3} k+c_{\mathbf{P}}^{\sigma_{j}}
$$

the following identity holds in distributional sense, for $\left\{\mathbf{k}:|\mathbf{k}| \leq \sigma_{j}\right\}$ :

$$
\begin{aligned}
& \left(\frac{1}{H_{\mathbf{P}, \sigma_{j}}^{w}-E_{j+1}}\right) b^{\dagger}(\mathbf{k}) \\
& =b^{\dagger}(\mathbf{k})\left(\frac{1}{\frac{1}{2 m}\left(\Gamma_{\mathbf{P}, \sigma_{j}}+\mathbf{k}\right)^{2}+\int|\mathbf{q}| \alpha_{\mathbf{P}}^{\sigma_{j}}(\widehat{\mathbf{q}}) b^{\dagger}(\mathbf{q}) b(\mathbf{q}) d^{3} q+|\mathbf{k}| \alpha_{\mathbf{P}}^{\sigma_{j}}(\widehat{\mathbf{k}})+c_{\mathbf{P}}^{\sigma_{j}}-E_{j+1}}\right)
\end{aligned}
$$

Moreover, for $\frac{\kappa}{m}$ sufficiently small, due to the prescription for $\left|E_{\mathbf{P}}^{\sigma_{j}}-E_{j+1}\right|$, due to the constraints on $\left|\nabla E^{\sigma_{j}}(\mathbf{P})\right|$, being $\sigma_{j+1} \leq|\mathbf{k}| \leq \sigma_{j}$ and taking into account $E_{\mathbf{P}}^{\sigma_{j}}-c_{\mathbf{P}}^{\sigma_{j}}$, the following bound holds:

$\left\|\left(\frac{1}{H_{\mathbf{P}, \sigma_{j}}^{w}+|\mathbf{k}| \alpha_{\mathbf{P}}^{\sigma_{j}}(\widehat{\mathbf{k}})-E_{j+1}}\right)^{\frac{1}{2}}\left(\frac{1}{m} \mathbf{k} \cdot \Gamma_{\mathbf{P}, \sigma_{j}}+\frac{\mathbf{k}^{2}}{2 m}\right)\left(\frac{1}{H_{\mathbf{P}, \sigma_{j}}^{w}+|\mathbf{k}| \alpha_{\mathbf{P}}^{\sigma_{j}}(\widehat{\mathbf{k}})-E_{j+1}}\right)^{\frac{1}{2}}\right\|_{F_{\sigma_{j}}^{+}} \leq \frac{1}{2}$.

Therefore the series expansion

$$
\left(\frac{1}{H_{\mathbf{P}, \sigma_{j}}^{w}+|\mathbf{k}| \alpha_{\mathbf{P}}^{\sigma_{j}}(\widehat{\mathbf{k}})-E_{j+1}}\right) \sum_{n=0}^{+\infty}\left(-\left(\frac{1}{m} \mathbf{k} \cdot \Gamma_{\mathbf{P}, \sigma_{j}}+\frac{\mathbf{k}^{2}}{2 m}\right) \frac{1}{H_{\mathbf{P}, \sigma_{j}}^{w}+|\mathbf{k}| \alpha_{\mathbf{P}}^{\sigma_{j}}(\widehat{\mathbf{k}})-E_{j+1}}\right)^{n}
$$

is well defined in $F_{\sigma_{j}}^{+}$.

Then we can write (note that $b(\mathbf{k}) \phi_{\mathbf{P}}^{\sigma_{j}}=0$ for $|\mathbf{k}| \leq \sigma_{j}$ ):

$$
\begin{aligned}
& \left(\int_{\sigma_{j+1}}^{\sigma_{j}} \frac{k^{i} b^{\dagger}(\mathbf{k})}{\sqrt{2}|\mathbf{k}|^{\frac{3}{2}} \alpha_{\mathbf{P}}^{\sigma_{j}}(\widehat{\mathbf{k}})} d^{3} k \Gamma_{\mathbf{P}, \sigma_{j}}^{i} \phi_{\mathbf{P}}^{\sigma_{j}},\left(\frac{1}{H_{\mathbf{P}, \sigma_{j}}^{w}-E_{j+1}}\right) \int_{\sigma_{j+1}}^{\sigma_{j}} \frac{k^{i} b^{\dagger}(\mathbf{k})}{\sqrt{2}|\mathbf{k}|^{\frac{3}{2}} \alpha_{\mathbf{P}}^{\sigma_{j}}(\widehat{\mathbf{k}})} d^{3} k \Gamma_{\mathbf{P}, \sigma_{j}}^{i} \phi_{\mathbf{P}}^{\sigma_{j}}\right) \\
& =\int_{\sigma_{j+1}}^{\sigma_{j}} \frac{k^{i^{2}}}{2|\mathbf{k}|^{3} \alpha_{\mathbf{P}}^{\sigma_{j}}(\widehat{\mathbf{k}})^{2}} \sum_{n=0}^{\infty} M_{n}(\mathbf{k}) d^{3} k
\end{aligned}
$$

where $M_{n}(\mathbf{k})$ corresponds to

$$
\left(\Gamma_{\mathbf{P}, \sigma_{j}}^{i} \phi_{\mathbf{P}}^{\sigma_{j}}, R_{H_{\mathbf{P}, \sigma_{j}}^{w}}^{\mathbf{k}}\left(E_{j+1}\right)\left(-\left(\frac{2 \mathbf{k} \cdot \Gamma_{\mathbf{P}, \sigma_{j}+} \mathbf{k}^{2}}{2 m}\right) R_{H_{\mathbf{P}, \sigma_{j}}^{w}}^{\mathbf{k}}\left(E_{j+1}\right)\right)^{n} \Gamma_{\mathbf{P}, \sigma_{j}}^{i} \phi_{\mathbf{P}}^{\sigma_{j}}\right)
$$

with $R_{H_{\mathbf{P}, \sigma_{j}}^{w}}^{\mathbf{k}}\left(E_{j+1}\right):=\left(\frac{1}{H_{\mathbf{P}, \sigma_{j}}^{w}+|\mathbf{k}| \alpha_{\mathbf{P}}^{\sigma_{j}}(\widehat{\mathbf{k}})-E_{j+1}}\right)$.

Exploiting the Schwartz inequality, we have that

$$
\left|\left(\Gamma_{\mathbf{P}, \sigma_{j}}^{i} \phi_{\mathbf{P}}^{\sigma_{j}}, R_{H_{\mathbf{P}, \sigma_{j}}^{w}}^{\mathbf{k}}\left(E_{j+1}\right)\left(-\left(\frac{2 \mathbf{k} \cdot \Gamma_{\mathbf{P}, \sigma_{j}+} \mathbf{k}^{2}}{2 m}\right) R_{H_{\mathbf{P}, \sigma_{j}}^{w}}^{\mathbf{k}}\left(E_{j+1}\right)\right)^{n} \Gamma_{\mathbf{P}, \sigma_{j}}^{i} \phi_{\mathbf{P}}^{\sigma_{j}}\right)\right|
$$


can be bounded by the product of the following quantities:

$$
\begin{aligned}
& \text { - }\left\|\left[\left(\frac{1}{H_{\mathbf{P}, \sigma_{j}}^{w}+|\mathbf{k}| \alpha_{\mathbf{P}}^{\sigma_{j}}(\widehat{\mathbf{k}})-E_{j+1}}\right)^{\frac{1}{2}}\right]^{\dagger} \Gamma_{\mathbf{P}, \sigma_{j}}^{i} \phi_{\mathbf{P}}^{\sigma_{j}}\right\|^{2} \\
& \text { - }\left\|\left(\frac{1}{H_{\mathbf{P}, \sigma_{j}}^{w}+|\mathbf{k}| \alpha_{\mathbf{P}}^{\sigma_{j}}(\widehat{\mathbf{k}})-E_{j+1}}\right)^{\frac{1}{2}}\left(\frac{1}{m} \mathbf{k} \cdot \Gamma_{\mathbf{P}, \sigma_{j}}+\frac{\mathbf{k}^{2}}{2 m}\right)\left(\frac{1}{H_{\mathbf{P}, \sigma_{j}}^{w}+|\mathbf{k}| \alpha_{\mathbf{P}}^{\sigma_{j}}(\widehat{\mathbf{k}})-E_{j+1}}\right)^{\frac{1}{2}}\right\|_{F_{\sigma_{j}}^{+}}^{n} .
\end{aligned}
$$

Hence, due to the estimate (a4) and Lemma 2.1, the absolute value of the scalar product

$$
\left(\int_{\sigma_{j+1}}^{\sigma_{j}} \frac{k^{i} b^{\dagger}(\mathbf{k})}{\sqrt{2}|\mathbf{k}|^{\frac{3}{2}} \alpha_{\mathbf{P}}^{\sigma_{j}}(\widehat{\mathbf{k}})} d^{3} k \Gamma_{\mathbf{P}, \sigma_{j}}^{i} \phi_{\mathbf{P}}^{\sigma_{j}},\left(\frac{1}{H_{\mathbf{P}, \sigma_{j}}^{w}-E_{j+1}}\right) \int_{\sigma_{j+1}}^{\sigma_{j}} \frac{k^{i} b^{\dagger}(\mathbf{k})}{\sqrt{2}|\mathbf{k}|^{\frac{3}{2}} \alpha_{\mathbf{P}}^{\sigma_{j}}(\widehat{\mathbf{k}})} d^{3} k \Gamma_{\mathbf{P}, \sigma_{j}}^{i} \phi_{\mathbf{P}}^{\sigma_{j}}\right)
$$

is bounded by

$$
\begin{aligned}
& \left\{\int_{\sigma_{j+1}}^{\sigma_{j}} \frac{k^{i^{2}}}{2|\mathbf{k}|^{3} \alpha_{\mathbf{P}}^{\sigma_{j}}(\widehat{\mathbf{k}})^{2}}\left\|\left(\frac{1}{H_{\mathbf{P}, \sigma_{j}}^{w}+|\mathbf{k}| \alpha_{\mathbf{P}}^{\sigma_{j}}(\widehat{\mathbf{k}})-E_{j+1}}\right)^{\frac{1}{2}} \Gamma_{\mathbf{P}, \sigma_{j}}^{i} \phi_{\mathbf{P}}^{\sigma_{j}}\right\|^{2} d^{3} k\right\} \cdot \sum_{n=0}^{\infty}\left(\frac{1}{2}\right)^{n} \\
& \leq 2 \int_{\sigma_{j+1}}^{\sigma_{j}} \frac{k^{i^{2}}}{2|\mathbf{k}|^{3} \alpha_{\mathbf{P}}^{\sigma_{j}}(\widehat{\mathbf{k}})^{2}} \cdot\left\|\left(\frac{1}{H_{\mathbf{P}, \sigma_{j}}^{w}+|\mathbf{k}| \alpha_{\mathbf{P}}^{\sigma_{j}}(\widehat{\mathbf{k}})-E_{j+1}}\right)^{\frac{1}{2}} \Gamma_{\mathbf{P}, \sigma_{j}}^{i} \phi_{\mathbf{P}}^{\sigma_{j}}\right\|^{2} d^{3} k \\
& \leq 2 \cdot Q(\epsilon) \cdot \int_{\sigma_{j+1}}^{\sigma_{j}} \frac{k^{i^{2}}}{2|\mathbf{k}|^{3} \alpha_{\mathbf{P}}^{\sigma_{j}}(\widehat{\mathbf{k}})^{2}} d^{3} k \cdot\left|\left(\Gamma_{\mathbf{P}, \sigma_{j}}^{i} \phi_{\mathbf{P}}^{\sigma_{j}},\left(\frac{1}{H_{\mathbf{P}, \sigma_{j}}^{w}-E_{j+1}}\right) \Gamma_{\mathbf{P}, \sigma_{j}}^{i} \phi_{\mathbf{P}}^{\sigma_{j}}\right)\right| .
\end{aligned}
$$

Collecting all the estimates the thesis follows.

Acknowledgments. The content of this paper is the first part of my P.H.D. dissertation in S.I.S.S.A, Trieste. I would like to thank G. Morchio and F. Strocchi for having suggested to me the problem and for discussions and advice. It is a pleasure to thank V. Bach, G. Dell'Antonio and J. Fröhlich for their interest in my work and for many enjoyable and interesting discussions. I have to very warmly thank J. Fröhlich for having explained to me some points of his old papers [Fr.1, Fr.2], for his helpful suggestions, criticism and advice. I am also grateful to C. Gerard, G.M. Graf and M. Griesemer for fruitful discussions.

\section{References}

[Ar] A. Arai, mp_arc 00-478 (2000).

[B.F.S] V. Bach, J. Fröhlich and I.M. Sigal, Adv. Math. 137(2), 299-395 (1998) and 137, 205-298 (1998).

[Bl] Ph. Blanchard, Comm. Math. Phys. 15, 156 (1969).

[Bu] D. Buchholz, Phys. Lett. B174, 331 (1986).

[Ch] T. Chen, preprint mp_arc 01-310 (2001).

[D.Ge] J. Derezinski and C. Gerard, Rev. Math. Phys 11, 383-450 (1999). 
[Fr.1] J. Fröhlich, Ann. Inst. H. Poincaré, Sect. A, XIX (1), 1-103 (1973).

[Fr.2] J. Fröhlich, Fort. der Phys. 22, 158-198 (1974).

[F.G.S] J. Fröhlich, M. Griesemer and B. Schlein, Los Alamos Preprint Archive, math-ph/0009033 (2000).

[F.G.S] J. Fröhlich, M. Griesemer and B. Schlein, math-ph/0103048 (2001).

[F.P] M. Fierz and W. Pauli, Nuovo Cimento 15, 167 (1938).

[Ge] C. Gerard, preprint mp_arc 99-158 (1999).

[Ge] C. Gerard, mp_arc 01-103 (2001).

[G.L.L] M. Griesemer, E.H. Lieb and M. Loss, preprint, Los Alamos Preprint Archive, math-ph/0007014 (2000).

[G.J] J. Glimm and A. Jaffe, Ann. Math. 91, 362 (1970).

[L.M.S] J. Lorinczi, A. Minlos and H. Spohn. Los Alamos Preprint Archive, math$\mathrm{ph} / 0011043(2000)$.

[Ne] E. Nelson, J. Math. Phys. 5, 1190-1197 (1964).

[Pi] A. Pizzo (in preparation).

[R.S] M. Reed, B. Simon, Methods of modern mathematical physics, Volumes 4, Academic Press, 1975.

[Sc] B. Schoer, Fortschr. Physik 11, 1-31 (1963).

[Sp] H. Spohn, J. Math. Phys. 38(5), 2281-2296 (1997).

\author{
Alessandro Pizzo \\ FB Mathematik \\ Johannes Gutenberg Universität \\ D-55099 Mainz, Germany \\ email: pizzo@mathematik.uni-mainz.de \\ Communicated by Gian Michele Graf \\ submitted 29/04/02, accepted 11/10/02
}

